\title{
Guidelines
}

\section{Evidence-Based Guideline of the German Nutrition Society: Fat Intake and Prevention of Selected Nutrition-Related Diseases}

\author{
Günther Wolfram $^{\mathrm{a}}$ Angela Bechthold $^{\mathrm{b}}$ Heiner Boeing ${ }^{c}$ Sabine Ellinger ${ }^{\mathrm{d}}$ \\ Hans Hauner ${ }^{\mathrm{e}}$ Anja Kroke ${ }^{\mathrm{f}}$ Eva Leschik-Bonnet ${ }^{\mathrm{b}}$ Jakob Linseisen ${ }^{\mathrm{g}}$ \\ Stefan Lorkowski ${ }^{\text {h }}$ Matthias Schulze ${ }^{c}$ Peter Stehle ${ }^{i}$ Jessica Dinter ${ }^{b}$
}

${ }^{\mathrm{a}}$ Technical University of Munich, Freising-Weihenstephan, ${ }^{\mathrm{b}}$ German Nutrition Society, Bonn, ${ }^{\mathrm{C}}$ German Institute of Human

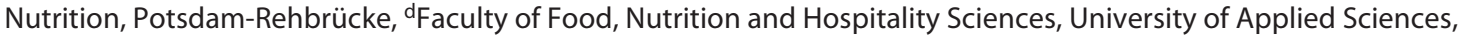
Niederrhein, Mönchengladbach, ${ }^{e}$ Else Kroener-Fresenius Center, Technical University of Munich, Munich, ${ }^{\mathrm{f}}$ Department of Nutritional, Food and Consumer Sciences, University of Applied Sciences, Fulda, IInstitute of Epidemiology, Helmholtz Centrum,

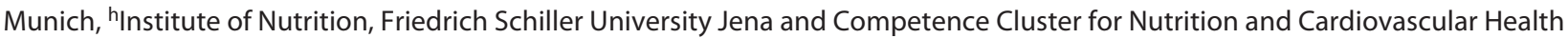
(nutriCARD), Jena-Halle-Leipzig, and 'Institute of Nutritional and Food Sciences, University of Bonn, Bonn, Germany

\section{Key Words}

Fat · Fatty acids · Prevention · Obesity · Type 2 diabetes mellitus · Dyslipoproteinaemia · Hypertension · Metabolic syndrome $\cdot$ Coronary heart disease $\cdot$ Stroke $\cdot$ Cancer

\footnotetext{
Abstract

As nutrition-related chronic diseases have become more and more frequent, the importance of dietary prevention has also increased. Dietary fat plays a major role in human nutrition, and modification of fat and/or fatty acid intake could have a preventive potential. The aim of the guideline of the German Nutrition Society (DGE) was to systematically evaluate the evidence for the prevention of the widespread diseases obesity, type 2 diabetes mellitus, dyslipoproteinaemia, hypertension, metabolic syndrome, coronary heart disease (CHD), stroke, and cancer through the intake of fat or fatty acids. The main results can be summarized as follows: it was concluded with convincing evidence that a reduced intake of total and saturated fat as well as a larger intake of
}

polyunsaturated fatty acids (PUFA) at the expense of saturated fatty acids (SFA) reduces the concentration of total and low-density lipoprotein cholesterol in plasma. Furthermore, there is convincing evidence that a high intake of trans fatty acids increases risk of dyslipoproteinaemia and that a high intake of long-chain polyunsaturated n-3 fatty acids reduces the triglyceride concentration in plasma. A high fat intake increases the risk of obesity with probable evidence when total energy intake is not controlled for (ad libitum diet). When energy intake is controlled for, there is probable evidence for no association between fat intake and risk of obesity. A larger intake of PUFA at the expense of SFA reduces risk of $\mathrm{CHD}$ with probable evidence. Furthermore, there is probable evidence that a high intake of long-chain polyunsaturated n-3 fatty acids reduces risk of hypertension and CHD. With probable evidence, a high trans fatty acid intake increases risk of $\mathrm{CHD}$. The practical consequences for current dietary recommendations are described at the end of this article.

\section{KARGER 125}

(c) 2015 S. Karger AG, Basel

0250-6807/15/0673-0141\$39.50/0

E-Mail karger@karger.com

www.karger.com/anm
Jessica Dinter

Department of Science, German Nutrition Society

Godesberger Allee 18

DE-53175 Bonn (Germany)

E-Mail dinter@dge.de 


\section{Introduction}

The German Nutrition Society's (DGE) evidence-based guidelines for the prevention of selected nutrition-related diseases reflect the current state of knowledge regarding whether, and in what way, a nutritional factor can affect the risk of disease. The evidence-based guideline 'Fat Intake and Prevention of Selected Nutrition-Related Diseases' was the first German Nutrition Society's guideline of its kind and was revised in January 2015 [1].

Due to its significance in prevention, the guideline examined the association between the quantity and quality of dietary fats and the risk of the following diseases:

- Obesity

- Type 2 diabetes mellitus

- Dyslipoproteinaemia

- Hypertension

- Metabolic syndrome

- Coronary heart disease (CHD)

- Stroke

- Cancer

Due to capacity constraints, it was not possible to consider all diseases for which fat intake may be relevant.

The issue of optimal fat intake has been discussed ever since recommendations for health-promoting nutrition were introduced, with the following properties of dietary fats playing a key role:

- Dietary fats are important sources of energy, thereby increasing the energy content and energy density of foods.

- Dietary fats contain fatty acids of various chain lengths and degrees of saturation which determine their different physical (melting point or fluidity of cell membranes) and chemical (e.g. process of chemical reactions) behaviour and their different biological functions (e.g. effect on plasma cholesterol concentration or effects derived from eicosanoids as metabolites of n-3 and n-6 fatty acids). It is therefore assumed that individual dietary fats may differently affect the risk of various diseases.

- Dietary fats are sources of fat-soluble vitamins as well as flavours and aromatics. They may also have a positive effect on the texture of food.

The revised guideline 'Fat Intake and Prevention of Selected Nutrition-Related Diseases' will provide professionals, intermediaries and the media with the latest science-based findings regarding the primary prevention of nutrition-related diseases through an appropriate intake of dietary fats.

\section{Methodological Approach}

\subsection{Key Question}

The key question for the guideline was 'How is the strength of evidence for a potential causal relation between quantity and quality of dietary fat and incidence of obesity, type 2 diabetes mellitus, dyslipoproteinaemia, hypertension, metabolic syndrome, CHD, stroke, and cancer?'

\subsection{Search Strategy}

A systematic literature search was conducted with a primary search focus on meta-analyses, systematic reviews and monographs. If these were not available, original papers on intervention and cohort studies were searched for. Case-control and cross-sectional studies were regarded as inappropriate evidence and were therefore not considered. The starting point of the literature search was the first version of the DGE guideline on fat intake; its search results were complemented by the results of this current search. The search period for original papers ranged from January 1, 2005 to June 30, 2012 (except for cancer, where single studies from 2013 were taken into account). For meta-analyses, the search was carried out up to October 27, 2014.

Only articles written in English or German were included. The literature search was conducted in the PubMed (www.ncbi.nlm. nih.gov/pubmed) and Cochrane (www.cochrane.org) databases and by reviewing reference lists of reviews and original papers.

For the literature search, the following key words were used:

- Obesity: obesity, overweight, weight(s), weight-gain, weightmaintenance, weight-control, weight-change, weight-loss, weight-reduction, caloric intake, body fat, waist circumference, energy intake, BMI, body mass index, body weight, obese, fat distribution, energy density

- Type 2 diabetes mellitus: diabetes, insulin sensitivity, insulin resistance, prediabetes, impaired glucose tolerance, impaired fasting glucose, diabetic

- Dyslipoproteinaemia: dyslipoprotein(a)emia, dyslipid(a)emia, hyperlipoprotein (a)emia, hypertriglycerid(a)emia, hyperlipid(a) emia, hypercholesterol(a)emia, hypercholesterin(a)emia, serum triglycerides, plasma triglycerides, serum lipids, plasma lipids, serum cholesterol, plasma cholesterol, LDL, HDL, lipoprotein(s), risk factor + cardiovascular, cholesterol(a)emic, hypercholesterol (a)emic, hyperlipoprotein (a)emic, dyslipoprotein(a)emic, hyperlipid(a)emic, dyslipid(a)emic, biomarker + cardiovascular

- Hypertension: blood pressure, hypertension, hypertonia, hypertensive

- Metabolic syndrome: metabolic syndrome, syndrome X, insulin resistance syndrome, deadly quartet

- CHD: cardiovascular disease, atherosclerosis, arteriosclerosis, $C V D$, coronary artery disease, coronary heart disease, CHD, myocardial infarction

- Stroke: stroke, ischemic, thrombotic, hemorrhagic, cerebrovascular disease

- Cancer: cancer, tumor, adenoma, polyp(s)

The key words used for each disease were combined with the following key words: fat, dietary fat, total fat intake, fatty acid, saturated fat, unsaturated fat, monounsaturated fat, polyunsaturated fat, cholesterol, n-3 fatty acid, omega-3 fatty acid, trans fat, CLA, conjugated linoleic acid, eicosapentaenic acid, eicosapentaenoic acid, docosahexaenic acid, docosahexaenoic acid, high-fat, low-fat. 
Fig. 1. Classification of levels of evidence (LOE).

\begin{tabular}{|cl|}
\hline LOE I & \\
Ia & Meta-analyses of randomised controlled intervention studies \\
Ib & Randomised controlled intervention studies \\
Ic & Non-randomised/non-controlled intervention studies (if well-designed) \\
\hline LOE II & \\
IIa & Meta-analyses of cohort studies \\
IIb & Cohort studies \\
\hline
\end{tabular}

Table 1. Criteria for judging the strength of evidence

\begin{tabular}{ll}
\hline Strength of evidence & Criteria for strength of evidence \\
\hline Convincing & $\begin{array}{l}\text { There is a considerable number of prospective cohort studies and, if possible, randomised controlled } \\
\text { intervention studies of sufficient size, duration and quality with consistent results (at least } 2 \text { randomised } \\
\text { controlled intervention studies of the highest quality (LOE I); in case of methodological weaknesses or } \\
\text { cohort studies only: at least } 5 \text { studies). Ideally, a recent meta-analysis is available (with neither } \\
\text { heterogeneous study results, nor high percentage of study results with opposite effects). }\end{array}$ \\
\hline Probable & $\begin{array}{l}\text { Epidemiological studies show consistent relations between factor and disease. However, there are noticeable } \\
\text { weaknesses regarding the causal argumentation or there is evidence for an opposite relation, which does not } \\
\text { allow a definite judgement. The required number of studies for classifying the strength of evidence as } \\
\text { 'probable' remains at as many as } 5 \text { very good studies (LOE I and/or LOE II). }\end{array}$ \\
\hline Possible & $\begin{array}{l}\text { There are only intervention studies, cohort studies or non-controlled clinical trials available, which were not } \\
\text { conducted to a sufficient standard. The results of most studies and at least of } 3 \text { studies point in the same } \\
\text { direction. Other studies without risk relations or with opposite risk relations may exist. }\end{array}$ \\
\hline $\begin{array}{l}\text { Only a few study results indicate an association between a factor and a disease, but they are not sufficient to } \\
\text { establish the association. This means the association between the nutritional factor and the disease has not } \\
\text { yet been or has rarely been investigated, or the studies available are inconsistent with a majority of studies } \\
\text { without risk relations and nearly equally as strong opposite results. }\end{array}$
\end{tabular}

If too many hits required a further limitation, the search was combined with the following terms: intake, uptake, consumption, $\operatorname{diet}(\mathrm{s})$, dietary, nutrition(al).

\subsection{Classification of the Literature into Levels of Evidence}

The literature identified with the systematic search was classified according to the levels of evidence I (intervention studies) and II (cohort studies) as shown in figure 1.

The results of the selected studies were recorded and systematically presented in tables, separately for each of the aforementioned disease outcomes (https://www.dge.de/wissenschaft/leitlinien; German only).

\subsection{Judgement of Strength of Evidence}

The strength of evidence was evaluated according to the judgement scheme of the World Health Organisation [2] following the criteria summarised in table 1 . The strength of evidence was divided into 4 categories: convincing, probable, possible and insufficient. The strength of evidence indicates the quality and consistency of the scientific basis for the described associations.

For judging the strength of evidence, controlled intervention studies were given a higher weight than cohort studies due to their experimental nature and their lower risk of biases. In addition, the number of studies, their quality and the biological plausibility of an association were taken into account.

The strength of evidence was elevated using the respective results tables and draft texts by consensus of all members of the guideline commission. In case of diverging judgements, a consensus was achieved by discussion.

\subsection{Consideration of Studies on Primary Prevention}

The revised guideline deals exclusively with studies on primary prevention. Studies on secondary prevention were considered only in the case of meta-analyses, which included studies on both primary and secondary prevention. In this respect, the revised guideline deviates from the first version, where both primary and secondary prevention were considered. However, it follows the same approach as the most recent DGE guideline on carbohydrate intake [3].

\subsection{Evaluation of Nutritional Data on Fat Intake}

Fat intake closely correlates with energy intake, which makes it difficult to separate the effects from each other. Due to the strong relation between energy and body mass, an effect of fat that goes beyond an energy-based effect can be postulated only when there is a risk relation for the energy-adjusted fat intake, controlled for body mass or relative body mass (BMI). By using such an isoenergetic 
model, the effect of the relative fat content of total energy intake can be examined irrespective of the energy balance. It has been shown that such a model can control the energy effect well [4]. Variations in fat intake can be achieved only by exchange with other energy sources, as this is the only way to keep energy intake constant. With this statistical model, an intervention study is simulated in which the proportion of fat of the total energy intake and the composition of fatty acids are replaced by other nutritional energy sources.

In addition to quantitative fat intake, the quality of fat may also affect disease risk. A suitable assessment model for such an effect is a distinction between the various fatty acids, adjusted for energy intake. If energy itself is included in the statistical model, the effects of fat intake are tested in an isocaloric state with stable body weights.

These considerations on nutritional parameters suitable for measuring the effects of fat intake on the risk of disease as well as the statistical models used for this have not yet been sufficiently incorporated in epidemiological practice. Further problems arising from the collection and analysis of nutritional data, such as measurement errors and selective under-reporting of certain components, are of a more general nature [5-7]. Therefore, the evaluation of the effect of fat from observational studies should always be viewed in consideration of the statistical models for deriving the risk estimates. It should be noted that the statistical models used for analysing an observational study can be regarded as an attempt to stimulate an intervention study. Statistical adjustment aims at achieving equality between groups, as it is achieved through randomisation applied in interventional studies. For example, a cohort study in which an adjustment was made for energy intake simulates an isocaloric intervention study.

In addition to the previously described qualitative and quantitative aspects of fat intake, dietary patterns and their association with fat intake and chronic diseases might also be considered. However, as this analytical approach does not allow any conclusions regarding the effects of fat intake alone, studies on dietary patterns were not included in this guideline.

Similarly, studies on fat containing foods (like nuts, meat, fish, milk, dairy products etc.) were not considered here, as the effects of foods are dependent on the entire food matrix and, as a result, do not allow to draw clear statements on the effects of either total fat, individual fatty acids or cholesterol.

\section{Quantity and Quality of Dietary Fat and Fatty Acid Intake and Primary Prevention of Obesity}

For the evaluation of studies regarding the significance of fat intake for the development of obesity, a distinction is made between different studies. There are studies in which the subject groups were supervised in the same way during the intervention with the aim of a consistent energy intake, or an energy adjustment was carried out with statistical modelling during observational studies. There are also studies that have allowed or targeted a change in energy intake (ad libitum) during an intervention, and observational studies that have created such a situation due to a lack of energy adjustment.
The separation of the effect of fat from the effect of energy in epidemiological observational studies such as cohort studies therefore takes place via energy adjustment, creating an isocaloric situation in the analysis of the effect of fat. This is synonymous with an intervention study carried out under isocaloric conditions. However, an isoenergetic situation can also be created in intervention studies carried out under ad libitum conditions through statistical modelling of the effect of energy. The studies evaluated according to this principle are also classified as isocaloric in this guideline. When energy adjustment is used, the effect of energy intake is therefore eliminated, whereas in the evaluation of ad libitum studies, it comes into effect and reflects the higher energy density of fat.

\subsection{Total Fat}

\section{Intervention Studies with Energy Control}

(Energy-Adjusted)

The intervention studies by Sheppard et al. [8] and Donnelly et al. [9] outlined below were carried out under ad libitum conditions. However, an isoenergetic situation was created using statistical modelling.

In the Women's Health Trial Feasibility Study, an intervention $(\mathrm{n}=171)$ with a low-fat diet (21 en $\%$ fat) parallel to a control group $(\mathrm{n}=105)$ with a normal diet $(38$ en\% fat) resulted in weight loss of $3 \mathrm{~kg}$ after 1 year and 1.9 $\mathrm{kg}$ in total after 2 years with almost constant weight $(-0.4$ $\mathrm{kg}$ ) in the control group. For every 1 -en\% lower fat content in the diet, a $0.1-0.25 \mathrm{~kg}$ lower body weight was observed. Weight loss was more closely associated with the proportion of fat of total energy than with the change in total energy intake [8] (LOE Ib).

In the study by Donnelly et al. [9] (LOE Ib) including 260 adults, the aim was to prevent a weight increase by reducing fat. A high-fat diet (40 en\%) caused a significant weight increase of $1 \mathrm{~kg}(\mathrm{p}<0.0366)$ after 12 weeks, whereas a low-fat diet (20 en\%) did not result in any significant weight increase. After adjustment for energy intake, the differences were no longer significant. In this study, weight increase was a function of energy intake and not of the proportion of fat of total energy.

\section{Observational Studies (Cohort Studies) with Energy Control (Energy-Adjusted)}

In a Swedish study of 308 women, an increased fat intake during the course of 6 years was only associated with an increase in body weight in women who had an overweight parent $(\mathrm{p}=0.003)$ [10] (LOE IIb). In a cohort of 
19,478 men aged between 40 and 70, there was a significant $(\mathrm{p}<0.003)$ positive association between fat intake, along with other factors such as snacks, and weight gain during the 4-year observation period. Per 10-gram increase in daily fat intake, an average weight increase of $100 \mathrm{~g}$ was observed [11] (LOE IIb). In a prospective cohort study over 14 years with 782 adults, there was a significant positive association ( $\mathrm{p}<0.0103)$ between en $\%$ of fat in the diet and an increase in weight [12] (LOE IIb).

In a large prospective cohort study (Nurses' Health Study) of 31,940 women, there was a positive association between weight gain and intake of animal fat, but not between weight gain and total fat intake after 8 years [13] (LOE IIb). In the same study, the observation of 41,518 women over 8 years found a weak positive association $(\beta=0.11)$ between total fat intake and increased body weight. The relation between animal fat and weight gain was stronger than the relation between total fat intake and weight gain. For overweight people, the relation between animal fat and weight increase was more pronounced than for those of normal weight [14] (LOE IIb).

A meta-analysis of 4 energy-adjusted cohort studies found no significant association, neither in men nor in women, between the proportion of fat of the total energy intake and an increase in body weight [15] (LOE IIa).

As part of a long-term (6 years) prospective study with 361 women, in a subgroup of 57 women with no appreciable physical activity and with a higher-fat diet $(\geq 38.5$ en $\%$ fat $)$, a significantly ( $p=0.03$ ) higher body weight was observed, $3.2 \mathrm{~kg}$ higher than with a lower-fat diet $(<38.5$ en\% fat). Following adjustment for energy intake, the difference was no longer significant. Women with more frequent physical activity did not exhibit this difference in body weight caused by fat intake [16] (LOE IIb).

In an older cohort study with 465 men and women over a period of 4 years, none of the energy-supplying nutrients were associated with an increase in body weight [17] (LOE IIb). In 6 cohorts of the prospective EPIC Study with 89,432 participants, there was no association between dietary fat (energy-adjusted amount and en\%) and body weight over 3.7-10 years. However, significant associations were found between fat intake and BMI (men: $p=0.0026$; women: $p=0.008)$. These were not observed in all national cohorts [18] (LOE IIb). A lower-fat diet (34.4 en\% fat) in the SUN Cohort ${ }^{1}$ with 4,556 participants over an observation period of more than 2 years was associated with a smaller weight increase compared to a

${ }^{1}$ SUN $=$ Seguimiento Universidad de Navarra.

Evidence-Based Guideline of the German Nutrition Society higher-fat diet (39.7 en\% fat). However, after adjustments were made, the difference was no longer significant [19] (LOE IIb). In the Danish cohort of the European Youth Heart Study with 384 9-year-old children, no relation could be determined between fat intake and a change in weight over the 6-year observation period [20] (LOE IIb).

Taking energy intake into consideration, the evidence regarding a lack of an association between fat intake and the risk of obesity is judged as probable. This is shown in both intervention and cohort studies.

\section{Intervention Studies without Energy Control (ad libitum)}

A series of controlled, randomised intervention studies examined the effect of reducing fat content in food on other chronic diseases. The changes in body weight in these studies are valuable for matters related to the primary prevention of obesity, as for these people the chronic disease is the focus and not body weight. In these studies, the control of energy intake also plays only a minor role.

In a larger meta-analysis, 37 controlled intervention studies lasting between 1 and 48 months with 11,586 participants were evaluated. The studies primarily examined the effect of Step I and Step II diets from the USA's $\mathrm{Na}$ tional Cholesterol Education Program (NCEP) on cardiovascular risk factors. In around one fifth of these studies, body weight was also a target value. There was a significant relation $(\mathrm{r}=0.46, \mathrm{p}<0.001)$ between the proportion of fat in the diet and body weight development, with a $1 \%$ reduction in the proportion of fat of total energy intake corresponding to a body weight decrease of $0.28 \mathrm{~kg}$ ( $\mathrm{p}<$ 0.0001 ). Thus, a reduced-fat diet will be more effective when the fat content is higher in the previous diet. The weight reduction was attributed to a spontaneous reduced energy intake in the fat-reduced diet, which was demonstrated by a significant correlation between weight loss and spontaneous reduction in energy intake $(r=0.54$, $\mathrm{p}<0.001)$ and its significant correlation with the reduction in dietary fat $(\mathrm{r}=0.47, \mathrm{p}<0.001)$. While in the intervention studies with unchanged physical activity, body weight decreased by an average of $2.79 \mathrm{~kg}$, in the studies with increased physical activity $(n=14)$ there was an average decrease of $5.66 \mathrm{~kg}$. The weight losses were significantly different $(\mathrm{p}<0.0001)$. Increased physical activity augmented weight loss from a fat-reduced diet and was also able to counteract the weight-increasing effect of a higher proportion of fat in the diet. The effect of a reduction in the proportion of fat in the diet on body weight 
was independent of the effect of increased physical activity [21] (LOE Ia).

In another meta-analysis, 16 intervention studies with 1,728 participants lasting between 2 and 12 months were analysed. The low-fat (3.5-24 en\%) ad libitum diet resulted in a greater weight loss of $2.4 \mathrm{~kg}(\mathrm{p}<0.0001)$ compared to the control groups with higher-fat diets, meaning that for every 1 -en $\%$ lower proportion of fat in the diet there was a weight decrease of $0.37 \mathrm{~kg}$. However, in a small proportion of studies in this meta-analysis, weight loss was intended. Excluding such studies from the evaluation did not significantly change the result [22] (LOE Ia). A further meta-analytical evaluation of an almost identical set of studies confirmed these results [23] (LOE Ia).

However, the meta-analyses performed by Yu-Poth et al. [21] (LOE Ia) and Astrup et al. [22, 23] (LOE Ia) also contain isolated studies with a planned energy deficit and with body weight as a target value, which may lead to the consequence that the extent of weight loss during a diet with a lower proportion of fat might be slightly overestimated.

In a more recent large meta-analysis, 33 intervention studies with 57,735 participants and duration of 6 months to 8 years were evaluated. At the end of each test period, the lower-fat diet led to a $1.57-\mathrm{kg}$ lower body weight on average ( $p<0.001$ ) compared to the diet with regular fat content, meaning that a $0.19-\mathrm{kg}$ lower body weight was observed per 1-en\% lower proportion of fat in the diet. A larger reduction in the proportion of fat was also accompanied by greater weight loss than was the case with a smaller reduction in the proportion of fat. In this metaanalysis, it is important to note that studies with the stated goal of weight reduction were excluded and that care was taken to ensure that the test and control groups had the same level of supervision and control [24] (LOE Ia). In a Cochrane Review of the same working group, a significantly lower body weight was determined for a reduced-fat diet than for a regular diet $(\mathrm{RR}=-0.83 ; 95 \% \mathrm{CI}$ -1.37 to 0.30$)$ and in terms of BMI, the RR was -0.47 (95\% CI -0.72 to -0.23 ) [25] (LOE Ia).

An intervention with the target value breast dysplasia in 29 women of normal weight with a fat-reduced ad libitum diet (26 en\% fat) resulted in a significantly lower body weight after 6 months, whereas in the control group (36 en $\%$ fat), the weight remained the same. After 1 year, however, the weight loss was no longer significantly different [26] (LOE Ib). In an isocaloric intervention study with 548 participants over the course of 24 weeks, a lower fat diet (28 en $\%$ fat) led to a weight loss of $1.1 \%(\mathrm{p}<0.001)$ compared to the higher fat diet (38 en\% fat). This weight loss correlated with the reduced energy intake $(\mathrm{p}<0.001)$ of the participants in the group with the lower-fat diet who did not adhere closely enough to the dietary (isocaloric) requirements [27] (LOE Ib).

\section{Observational Studies (Cohort Studies) without Energy Adjustment}

Nowadays, energy is generally considered as a separate variable in the statistical modelling of the effects of fat. Nonetheless, there are still studies in the literature with tables listing risk estimates for fat in the development of obesity without energy adjustment. This information is presented here.

In a large prospective study $(n=12,669)$ over almost 6 years, the relative risk of a weight increase of over $5 \mathrm{~kg}$ in 5 years for women in the top quintile of fat intake was 1.7 (95\% CI 1.1-2.7). This relation could not be confirmed in men [28] (LOE IIb).

In the Pound of Prevention Study of 1,044 men and women with annual measurements of body weight, dietary behaviour and activity level over 3 years, there was a positive correlation between fat in the diet (in absolute as well as percentage terms) and an increase in weight [29] (LOE IIb).

In the Quebec Family Study, 248 participants were observed over the course of 6 years. Individuals who consumed a lower proportion of fat and fewer high-fat foods, yet had a tendency towards a higher fibre intake $(+1.1 \mathrm{~g}$ of fibre per 1,000 kcal), spontaneously exhibited a lower energy intake and a significantly $(\mathrm{p}<0.05)$ lower increase in body weight, body fat percentage and skinfold thickness during the course of the study [30] (LOE IIb). In a cohort of 879 children and adolescents (Viva la Familia Study), there was a positive association between the proportion of fat of total energy intake and weight gain. This relation was more pronounced in overweight children [31] (LOE IIb).

The results of the ad libitum intervention studies as well as the cohort studies without energy adjustment show with probable evidence that there is a positive association between the proportion of fat in the diet and the risk of obesity.

In several of the studies mentioned earlier on the influence of the amount of fat in the diet on body weight, the influence of certain fatty acids, and therefore the quality of dietary fat, was also examined. In the following section, the results on fatty acids are presented. Additional information about the respective studies can be found in the brief descriptions of these studies in this section 3.1. 


\subsection{Saturated Fatty Acids}

\section{Studies with Energy Adjustment}

An effect of a high intake of saturated fatty acids (SFA) which favours the development of overweight was observed in the cohort study by Field et al. [14] (LOE IIb). In the cohorts of the EPIC Study, a weak, inverse association between SFA intake and body weight was observed for women, but not for men [18] (LOE IIb). In the Heidelberg cohort of the EPIC Study, individual SFA were also examined in 7,640 men and women of normal weight with 6.5 years of follow-up. In this study, there was a significant linear association between intake of stearic acid $(18: 0)$ and weight increase in men $(\mathrm{p}<0.03)$ and women $(\mathrm{p}<0.01)$, but there was no association between intake of palmitic acid (16:0) and weight increase [32] (LOE IIb).

For an association between the proportion of SFA in the diet and the primary prevention of obesity, the evidence in studies with energy adjustment is judged as insufficient.

Studies without Energy Adjustment

In the intervention study of Bo et al. [33] (LOE Ic), a positive association between SFA intake and weight gain was observed. In the cohort study of Butte et al. [31] (LOE IIb), however, it was not.

For an association between the proportion of SFA in the diet and the primary prevention of obesity, the evidence in studies without energy adjustment is judged as insufficient.

\subsection{Monounsaturated Fatty Acids}

\section{Studies with Energy Adjustment}

As part of an intervention in 162 healthy subjects where the proportion of fat was increased from $33-37 \%$ of total energy, a high-monounsaturated fatty acids (MUFA) diet in comparison with a high-SFA diet was not associated with changes in BMI after 3 months of isoenergetic nutrition [34] (LOE Ib).

A higher consumption of olive oil or oleic acid was not associated with an increase in weight in 2 cohort studies $[14,35]$ (LOE IIb). In 6 cohorts of the EPIC Study, there was no association between the level of MUFA intake and changes in body weight [18] (LOE IIb). In the Heidelberg cohort of the EPIC Study, the intake of oleic acid was not associated with changes in body weight in the linear mod- el. In the multinomial model, there was a significantly reduced risk of weight increase with increasing oleic acid intake for women with a small weight increase $(\mathrm{p}=0.02)$ and for men with a large weight increase $(\mathrm{p}=0.04)$ [32] (LOE IIb). In a Spanish cohort study $(\mathrm{n}=340)$, the risk of overweight was 2.3 times higher $(\mathrm{p}<0.03)$ with the consumption of sunflower oil than with the consumption of olive oil after 6 years [36] (LOE IIb).

For the primary prevention of obesity through a diet with an increased proportion of MUFA, there is possible evidence for no association in studies with energy adjustment.

\section{Studies without Energy Adjustment}

In a meta-analysis carried out by Schwingshackl et al. [37] (LOE Ia), the significant difference in average body weight in 7 studies comparing a high-MUFA diet with a low-MUFA diet was only due to the significantly large difference in one individual study where almonds were used to increase MUFA intake. The bioavailability of fat from nuts is limited; for this reason, the result of a lower body weight in this case may have more to do with an energy deficit than with the intake of MUFA.

In the isocaloric RISCK Study ${ }^{2}$, a diet with low fat content compared to a diet with high MUFA content was associated with a decrease in body weight. The weight decrease correlated with a reduced energy intake of the participants in the group with the low fat content who did not adhere closely enough to the dietary (isocaloric) instructions [27] (LOE Ib).

In a crossover comparison of a low-fat (20 en $\%$ fat, 8 en\% MUFA) with a high-fat and high-MUFA (40 en\% fat, 26 en\% MUFA) ad libitum diet in 11 people, each for a duration of 6 weeks, the low-fat diet caused a significant weight decrease of $1.53 \mathrm{~kg}( \pm 1.21 \mathrm{~kg})$, whereas the highfat and high-MUFA diet did not [38] (LOE Ib).

In the Viva la Familia Study, a higher intake of MUFA was associated with a weight increase $(\mathrm{p}<0.05)$ [31] (LOE IIb).

For the primary prevention of obesity through a diet with an increased proportion of MUFA, the evidence is judged as insufficient in studies without energy adjustment.

${ }^{2}$ RISCK Study: The name of the study comes from the initials of the participating study centres. These are Reading, Imperia, Surrey, Cambridge and Kings. 


\subsection{Polyunsaturated Fatty Acids}

\subsubsection{Total Polyunsaturated Fatty Acids}

Studies with Energy Adjustment

In a Nurses' Health Study evaluation, intake of polyunsaturated fatty acids (PUFA) was not associated with weight increase [14] (LOE IIb). In the Heidelberg cohort of the EPIC Study, there was a significant association in the linear model between intake of linoleic and arachidonic acid in women and an increase in body weight ( $p=0.00$ and $p=0.02$ ). In the multinomial model, the higher intake of linoleic acid in women was also significantly associated with weight increase. For arachidonic acid, however, there was only a significant association in women with a small weight increase ( $\mathrm{p}=$ 0.01 ). In men, there was a significant positive association with weight increase in the linear model for higher intake of arachidonic acid ( $\mathrm{p}=0.01)$, but not for higher intake of linoleic acid. The increased intake of linoleic acid was associated with an increase in body weight $(\mathrm{p}=0.03)$ in the multinomial model for men with a large weight increase; there were no significant associations in the multinomial model regarding the level of arachidonic acid intake [32] (LOE IIb). In the cohorts of the EPIC Study, there was no association between PUFA intake and an increase in body weight [18] (LOE IIb).

Based on studies with energy adjustment and a biomarker study, for primary prevention of obesity through a diet with a moderate proportion of PUFA, the evidence for a positive association in women is judged as possible; for men, the evidence for a lack of an association is judged as possible.

\subsection{2 n-6 Fatty Acids}

Study without Energy Adjustment

In the Viva la Familia Study, there was a positive association between en $\%$ from n-6 fatty acids and weight increase $(\mathrm{p}<0.05)[31]$ (LOE IIb).

\section{Biomarker Study}

In the Danish Diet, Cancer and Health cohort (over 27,000 men and over 29,000 women aged between 50 and 64 ), in the adipose tissue of 1,100 women and men, a positive association was found between a fatty acids pattern with high n-6 fatty acid content and both body weight $(\mathrm{p}=0.036)$ and waist size in women, but not in men, following a median observation period of 5.4 years [39] (LOE IIb).
Based on studies without energy adjustment and a biomarker study, there is insufficient evidence for an association between the proportion of n- 6 fatty acids in the diet and the primary prevention of obesity.

\subsection{3n-3 Fatty Acids}

Study with Energy Adjustment

In the Heidelberg cohort of the EPIC Study, there was no significant association in both women and men between the level of $\alpha$-linolenic acid (ALA), eicosapentaenoic acid (EPA) and docosahexaenoic acid (DHA) intake and an increase in body weight [32] (LOE IIb).

\section{Study without Energy Adjustment}

In the Viva la Familia Study, the proportion of n-3 fatty acids of energy intake was positively associated with weight increase $(\mathrm{p}<0.05)$ [31] (LOE IIb).

\section{Biomarker Study}

In an analysis of biomarkers as part of the EPIC Study (1,998 men and women observed over 4.9 years), the proportion of long-chain n-3 fatty acids in plasma phospholipids was not associated with changes in body weight [40] (LOE IIb).

For the primary prevention of obesity through a diet with an increased proportion of n-3 fatty acids, the evidence in studies both with and without energy adjustment and based on a biomarker study is judged as insufficient.

\subsection{Trans Fatty Acids}

\section{Studies with Energy Adjustment}

In the Nurses' Health Study, a positive association was found between the intake of trans fatty acids and weight increase in women [13] (LOE IIb), which was confirmed in a later evaluation of the Nurses' Health Study [14] (LOE IIb).

\section{Biomarker Study}

In the Danish Diet, Cancer and Health cohort (over 27,000 men and over 29,000 women aged 50-64), in the adipose tissue of 1,100 women and men, a positive association was found between a pattern of fatty acids high in trans fatty acids and both body weight $(\mathrm{p}=0.037)$ and waist circumference $(\mathrm{p}=0.014)$ in women [39] (LOE IIb). 
For the primary prevention of obesity through a diet with a reduced proportion of trans fatty acids, the evidence based on studies with energy adjustment and a biomarker study is judged as possible.

\section{Study without Energy Adjustment}

In the Viva la Familia Study, no association was found between intake of trans fatty acids and body weight [31] (LOE IIb).

For the primary prevention of obesity through a diet with a reduced proportion of trans fatty acids, the evidence based on studies without energy adjustment is judged as insufficient.

\subsection{Other}

\subsubsection{Conjugated Linoleic Acids}

A meta-analysis of a total of 18 intervention studies on the effect of conjugated linoleic acids (CLA) on the body fat mass of normal and overweight people showed that, with an average dose of $3.2 \mathrm{~g}$ CLA per day as a supplement, fat mass could be reduced by $0.05 \mathrm{~kg}$ per week $(\mathrm{p}<$ 0.001 ). The individual studies were carried out with supplements and generally had a maximum duration of 12 weeks. Only 1 study lasted for 2 years, although it was not placebo-controlled in the second year. The decrease in body fat mass in the first 6 months was approximately linear [41] (LOE Ia). It is currently not possible to make statements regarding the opportunities and risks involved in the long-term administration of CLA.

There is convincing evidence for a practically insignificant decrease in body fat mass through short-term supplementation with CLA.

There is insufficient evidence for the primary prevention of obesity through a long-term diet with an increased proportion of CLA.

\subsubsection{Medium-Chain Triglycerides}

Studies with Energy Adjustment

For 24 men with a BMI of 28 in an intervention study, an isocaloric diet with 40 en $\%$ fat (65\% of which were medium-chain triglycerides (MCT)) led to an increase in energy turnover and fat oxidation and, after 4 weeks, to weight reduction, a significant decrease in total $(0.83 \mathrm{~kg}, \mathrm{p}<0.05)$ and subcutaneous $(-0.54 \mathrm{~kg}, \mathrm{p}<$ 0.05 ) adipose tissue and the adipose tissue in the upper part of the body $(-0.67 \mathrm{~kg}, \mathrm{p}<0.05)$ which was mea- sured with magnetic resonance tomography. After 4 weeks of the control diet, also with $40 \mathrm{en} \%$ fat (of which $75 \%$ was olive oil), these significant differences in the named parameters compared with baseline were no longer present [42] (LOE Ib). However, the advantages related to energy balance were already no longer able to be verified by adaptation mechanisms in the 2nd-4th week of application $[43,44]$ (LOE Ib). As MCT lead to intolerances in larger quantities ( $>60 \mathrm{~g} /$ day), a maximum of $80-120 \mathrm{kcal}$ can be saved per day through the use of MCT. Long-term intervention studies could not be identified.

The evidence for a temporary decrease in body fat mass through a short-term supplementation with MCT is judged as insufficient.

On the basis of studies with energy adjustment, the evidence for the long-term effectiveness of MCT in the primary prevention of obesity is judged as insufficient.

\subsection{Need for Research - Obesity}

Research into additional determinants of weight stability in people of normal weight is very important for the primary prevention of obesity. Determinants for weight maintenance following weight reduction are equally important.

The successes of the existing long-term intervention studies regarding the primary prevention of obesity through a diet with lower fat intake should be reinforced by additional intervention studies into effectiveness and safety, carried out on larger groups of healthy people over a period as long as possible. In particular, the effectiveness of the moderate fat diet, the intake of dietary fibre and the increased physical activity must be distinguished from one another.

To validate the prevention of obesity through a diet with a high proportion of fat, methodologically sound intervention studies into effectiveness and safety lasting significantly longer than 1 year and with a sufficiently large number of participants would be necessary.

In studies with both reduced-fat diets and high-fat diets, the effects of different fatty acids (SFA, MUFA or PUFA) and different carbohydrates (sugars, polysaccharides), the quantity and type of dietary fibres and also the quantity and type of protein require further clarification. At the same time, biomarkers for the supply of essential nutrients and metabolic imbalances (e.g. ketone bodies) must be observed. 
Special efforts are required towards generally improving compliance regarding the prevention of obesity through suitable nutrition and lifestyle changes.

\section{Quantity and Quality of Dietary Fat and Fatty Acid Intake and Primary Prevention of Type 2 Diabetes Mellitus}

\subsection{Total Fat}

In several randomised, controlled intervention studies it was verified that a lifestyle intervention focused on dietary change and weight reduction can lower the conversion rate of an impaired glucose tolerance to type 2 diabetes mellitus. In the Finnish Diabetes Prevention Study, 522 overweight subjects (average BMI: 31 ) received either a short, standard consultation or intensive, personalised instructions for weight loss, health-conscious nutrition and increased physical activity. In addition to a reduction in the proportion of total fat intake to below $30 \%$ of total energy intake, the dietary recommendations also included an increase in dietary fibre intake [45] (LOE Ib). In the Diabetes Prevention Program, 3,234 subjects with impaired glucose tolerance were recruited and, following randomisation, were also provided with an intensive consultation program for dietary modification and physical activity. The dietary recommendations here also included a reduction in the proportion of total fat intake to below $30 \%$ of energy [46] (LOE Ib). After an intervention period of 3.2 and 2.8 years, respectively, both studies observed a $58 \%$ reduction in the progression from impaired glucose tolerance to type 2 diabetes mellitus [45, 46] (LOE Ib). In the Indian Diabetes Prevention Program [47] (LOE Ib), the risk of type 2 diabetes mellitus was also able to be lowered by a multifactorial lifestyle change, which implied a reduction in fat intake. The same observation was made in the Da Quin Study, a group-randomised intervention study [48] (LOE Ic). However, the extent to which the changes in fat intake influenced the result and whether total fat intake had an effect independent of the achieved weight loss remained unclear in the studies. This also applies to the older Malmö Prevention Study, a non-randomised intervention study in which a dietary intervention to reduce fat in conjunction with increased physical activity caused a significant decrease in incidences of diabetes in individuals with impaired glucose tolerance [49] (LOE Ic). In a New Zealand study, the proportion of diabetics after 1 year of intervention with a reduced-fat ad libitum diet was significantly lower than in the control group without intervention [50] (LOE Ib). The intervention led to a reduction in energy intake and body weight in this case, too. Differences between the study groups during the follow-up after completion of the intervention could not be verified.

In the Women's Health Initiative Dietary Modification Trial (WHI) including 45,887 postmenopausal women, women in the intervention group were motivated to reduce their fat intake. The intervention resulted in an increase in the proportion of carbohydrates compared to the control group ( 58.5 vs. 48.0 en\% after year 1; 54.1 vs. 45.9 en\% after year 6), largely at the expense of the proportion of fat ( 24.2 vs. 35.0 en $\%$ after year $1 ; 28.6$ vs. 36.9 en $\%$ after year 6). Women in the intervention group reduced their body weight in the first year of the study slightly more than the women in the control group (average body weight 74.0 vs. $75.9 \mathrm{~kg}$ ); after 6 years, there was only a small difference (75.6 vs. $76.2 \mathrm{~kg}$ ). There was no difference between the intervention and control group with regard to the incidence of type 2 diabetes mellitus over 9 years of follow-up $(\mathrm{RR}=$ 0.96; 95\% CI 0.90-1.03) [51] (LOE Ib).

In some older cohort studies, a positive association could be observed between total fat intake and the incidence of diabetes $[52,53]$ (LOE IIb). In the Finnish and Dutch cohort of the Seven Countries Study, the association between fat intake and the development of diabetes was investigated over an observation period of 20 years. Men with newly diagnosed type 2 diabetes mellitus had a higher fat intake at the beginning of the study $(n=26,41.2$ en $\%$ fat) than men with normal glucose tolerance ( $\mathrm{n}=$ $241,38.7$ en $\%$ fat). In this analysis, adjustments were made for age and cohort, but not for body weight and other important lifestyle characteristics or nutritional exposure [53] (LOE IIb). In the San Luis Valley Diabetes Study, people who developed type 2 diabetes mellitus over the 1 - to 3-year observation period $(n=20)$ had a higher fat intake of on average $43.4 \mathrm{en} \%$ compared to people who continued to have an impaired glucose tolerance ( $n=43$, $40.6 \mathrm{en} \% \mathrm{fat}$ ) and people whose glucose tolerance normalised ( $n=60,38.9 \mathrm{en} \%$ fat). The study was adjusted for age, sex and ethnic affiliation [52] (LOE IIb).

In more recent cohort studies, no such association could be found. In the Nurses' Health Study (84,204 women, 14 years of follow-up, 2,507 new diseases), the relative risk for the development of type 2 diabetes mellitus in the highest quintile of total fat intake (46.1 en\%) compared to the lowest quintile (28.9 en\%) was 0.97 (95\% CI 0.85-1.11) [54] (LOE IIb). In a more recent analysis of the cohort with extended follow-up (20 years, 4,670 new diseases), there was also no association between total fat intake and 
risk of diabetes. For a comparison of the highest decile of fat intake with the lowest, the relative risk was 0.91 (95\% CI 0.79-1.06) [55] (LOE IIb). The Iowa Women's Health Study, in which 35,988 women were observed over 11 years (1,890 new diseases), also arrived at a similar result. Here the relative risk for the comparison of extreme quintiles of total fat intake was 0.89 (95\% CI 0.75-1.05) [56] (LOE IIb). In the Health Professionals Follow-Up Study, the relative risk for the comparison of extreme quintiles was 0.97 (95\% CI 0.79-1.18), with 42,504 men observed in the study over 12 years (1,321 new diseases) [57] (LOE IIb). In the Kuopio Ischaemic Heart Disease Risk Factor Study, the total fat intake in people who developed type 2 diabetes mellitus ( $\mathrm{n}=34$ ) was not significantly different from people without the disease [58] (LOE IIb). In the EPIC-Norfolk Study (23,631 men and women, 3-7 years of follow-up, 414 new diseases), no association between total fat intake and risk of diabetes could be observed $(\mathrm{RR}=1.00$; $95 \% \mathrm{CI} 0.98$ 1.02) [59] (LOE IIb). Likewise, a comparison of people who consumed $<30 \mathrm{en} \%$ total fat with people who consumed a higher percentage of fat as a proportion of energy intake showed no statistically proven association in this cohort ( $\mathrm{RR}=1.28 ; 95 \%$ CI $0.98-1.68)$ [60] (LOE IIb). In a post hoc analysis of the Finnish Diabetes Prevention Study, in which all participants were pooled without consideration of group membership of the actual intervention study, an elevated risk was observed with higher total fat intake [61] (LOE IIb). The relative risk in the highest quartile of total fat intake ( $>36.86$ en\% fat) compared to the lowest quartile ( $<30$ en\% fat) was 2.14 (95\% CI 1.16-3.92). However, following adjustment for dietary fibre intake - a key aspect of the intervention along with total fat content - no significant association could be observed ( $\mathrm{RR}=1.23$; $95 \% \mathrm{CI}$ 0.95-1.58). In the Melbourne Collaborative Cohort Study $(3,737$ men and women, 4 years of follow-up), no association could be observed between total fat intake and the risk of diabetes (RR highest quintile compared to the lowest quintile $=1.12$; 95\% CI 0.76-1.73) [62] (LOE IIb). In the EPIC-Potsdam Study (25,067 men and women, 7 years of follow-up, 844 new diseases), an isocaloric substitution of fat with carbohydrates was not associated with the risk of diabetes [63] (LOE IIb).

The available data allow us to conclude that type $2 \mathrm{di}$ abetes mellitus cannot be prevented by a reduction of total fat intake, regardless of the effect this has on body weight. The majority of cohort studies, particularly those with large numbers of cases and control for confounding, as well as a large randomised intervention study, show that there is probable evidence for a lack of an association.

Evidence-Based Guideline of the German Nutrition Society

\subsection{SFA}

A reduction in the intake of SFA to a maximum of $10 \%$ of total energy intake was part of the interventions carried out in the Finnish Diabetes Prevention Study and the American Diabetes Prevention Program [45, 46] (LOE Ib). In both studies, the risk of developing type 2 diabetes mellitus was lowered by $58 \%$. Due to the multifactorial nature of the studies (weight loss, physical activity, change in other nutritional parameters, particularly total fat intake), it is not possible to attribute the observed effect to the change in SFA intake.

In a subgroup of the Seven Countries Study, a higher intake of SFA was observed at the beginning of the study in men with newly diagnosed type 2 diabetes mellitus than in men with normal glucose tolerance (21.6 en\% SFA compared to 20.1 en\% SFA) [53] (LOE IIb). In the San Luis Valley Diabetes Study, individuals with incidental type 2 diabetes mellitus did not have a significantly higher intake of SFA (16.1 en\%) than adults with normal glucose tolerance (14.0 en\%, p = 0.06) [52] (LOE IIb). In the Nurses' Health Study the relative risk of diabetes in the highest quintile (18.8 en\%) compared to the lowest quintile (10.7 en\%) of SFA intake was 0.99 (95\% CI 0.80-1.21), when adjusted for BMI, additional confounder and other fatty acids [54] (LOE IIb). In the Iowa Women's Health Study there was also no observed relation between SFA intake and the risk of diabetes (RR for extreme quintiles = 1.00; 95\% CI 0.85-1.18) [56] (LOE IIb). The Health Professionals Follow-Up Study showed no relation between SFA intake and the risk of diabetes (RR for extreme quintiles $=0.97 ; 95 \%$ CI 0.79-1.20) [57] (LOE IIb). In the EPIC-Norfolk Study, no significant association between SFA intake and risk of diabetes could be observed $(\mathrm{OR}=$ 1.03 for men, OR = 1.04 for women) [59] (LOE IIb). In the same cohort, however, people with an SFA intake $<10$ en\% compared to people with a higher SFA intake exhibited a reduced risk of diabetes $(\mathrm{RR}=0.71 ; 95 \%$ CI $0.51-$ 0.99) [60] (LOE IIb). A meta-analysis of the Nurses' Health Study, Iowa Women's Health Study, Health Professionals Follow-Up Study and the EPIC-Norfolk Study showed that no association existed between SFA intake and incidence of diabetes $(\mathrm{RR}=0.98 ; 95 \% \mathrm{CI} 0.87-1.10)$ [64] (LOE IIa).

In the Kuopio Ischaemic Heart Disease Risk Factor Study, intake of SFA in individuals who developed type 2 diabetes mellitus was not significantly different from people who did not develop diabetes [58] (LOE IIb). In the Women's Health Study, the relative diabetes risk in the highest quintile $(25.8 \mathrm{~g} /$ day $)$ compared to the lowest

Ann Nutr Metab 2015;67:141-205 151 
quintile ( $13.8 \mathrm{~g} /$ day) of SFA intake was 0.89 (95\% CI 0.66 1.21) when adjusted for BMI, additional confounder and total fat intake [65] (LOE IIb). In a post hoc analysis of the Finnish Diabetes Prevention Study, SFA intake was not significantly associated with the risk of diabetes. The relative risk in the highest quartile of SFA intake compared to the lowest quartile was 1.73 (95\% CI 0.89-3.38) [61] (LOE IIb). In the Melbourne Collaborative Cohort Study (3,737 men and women, 4 years of follow-up), no association could be observed between intake of SFA and the risk of diabetes ( $\mathrm{RR}$ highest quintile compared to the lowest quintile $=1.04 ; 95 \%$ CI 0.68-1.58) [62] (LOE IIb). In the EPIC-Potsdam Study, a higher intake of SFA was not associated with the risk of diabetes, when intake of SFA in exchange for carbohydrates was observed [63] (LOE IIb).

In the Iowa Women's Health Study, the substitution of SFA with PUFA was associated with a significantly lower risk of type 2 diabetes mellitus (RR for extreme quintiles $=0.84 ;$ 95\% CI 0.71-0.98) [56] (LOE IIb). An isoenergetic substitution of SFA with PUFA was linked to a $35 \%$ reduction in risk in the Nurses' Health Study (RR per 5 en $\%=0.65 ; 95 \%$ CI 0.54-0.78) [54] (LOE IIb). In the EPIC-Norfolk Study too, a higher ratio of PUFA to SFA was associated with a reduced risk of diabetes. However, this association was no longer significant following adjustment for anthropometric parameters $(\mathrm{RR}=0.91 ; 95 \%$ CI 0.81-1.03) [59] (LOE IIb). In the EPIC-Potsdam Study, a higher intake of SFA was associated with a reduced risk of diabetes, when SFA were considered at the expense of other fatty acids (RR highest quintile in comparison with the lowest quintile $=0.71 ; 95 \%$ CI 0.50-0.99) [66] (LOE IIb).

Cohort studies on whether an association exists between SFA intake and the risk of type 2 diabetes mellitus were unable to confirm this in the majority of cases. The evidence for a lack of an association is therefore judged as probable. The evidence that the substitution of SFA with PUFA lowers the risk of type 2 diabetes mellitus is judged as insufficient due to the low number of studies and their heterogeneity.

\subsection{MUFA}

Results from the Finnish and Dutch cohorts of the Seven Countries Study show that men with newly diagnosed type 2 diabetes mellitus exhibited a higher intake of MUFA (14.5 en\%) at the beginning of the study than men with normal glucose tolerance $(13.6$ en $\%, \mathrm{p}<0.05)$ [53] (LOE IIb). In the San Luis Valley Diabetes Study, people with glucose intolerance who developed type 2 diabetes mellitus had consumed a higher amount of MUFA (17.1 en\%) than people whose glucose tolerance normalised over the course of the observation period ( $14.9 \mathrm{en} \%, \mathrm{p}=$ 0.03) [52] (LOE IIb). In the more recent cohort studies Iowa Women's Health Study [56] (LOE IIb), Nurses' Health Study [54] (LOE IIb), Health Professionals FollowUp Study [57] (LOE IIb), Kuopio Ischaemic Heart Disease Risk Factor Study [58] (LOE IIb), Women's Health Study [65] (LOE IIb), Melbourne Collaborative Cohort Study [62] (LOE IIb) and the EPIC-Potsdam Study [63, 66] (LOE IIb), no association could be observed between intake of MUFA and risk of type 2 diabetes mellitus.

Despite positive relations in older, smaller cohort studies and due to the lack of association in large cohort studies, the evidence for a lack of an association between MUFA intake and the risk of type 2 diabetes mellitus is judged as probable.

\subsection{PUFA}

\subsubsection{Total PUFA}

In the San Luis Valley Diabetes Study, no relation could be observed between intake of PUFA and development of type 2 diabetes mellitus. In this study, people who developed type 2 diabetes mellitus did not have different intakes of PUFA (7.5 en\%) when compared with people who retained an impaired glucose tolerance $(6.8$ en\%) or returned to a normal glucose tolerance (7.0 en \%, $\mathrm{p}=0.74$ ) [52] (LOE IIb). In the Seven Countries Study too, people who developed type 2 diabetes mellitus did not have a significantly different PUFA intake (4.3 en\%) than people who retained a normal glucose tolerance ( 4.2 en $\%, p=0.81$ ) [53] (LOE IIb). In the Iowa Women's Health Study, after an 11-year observation period, there was a negative relation between PUFA intake and incidence of diabetes. However, this did not reach statistical significance (RR for extreme quintiles $=0.88 ; 95 \% \mathrm{CI}$ $0.76-1.02)$. In this analysis, no adjustment was made for other energy-supplying nutrients [56] (LOE IIb). In an evaluation of the Nurses' Health Study, there was a negative correlation between the proportion of PUFA of energy intake at the expense of carbohydrates and the risk of diabetes after controlling for various confounding factors $(\mathrm{RR}$ for extreme quintiles $=0.75 ; 95 \%$ CI $0.65-0.88$ ) [54] (LOE IIb). In the Kuopio Ischaemic Heart Disease 
Risk Factor Study, the intake of PUFA in people who developed type 2 diabetes mellitus was not significantly different from people who remained healthy [58] (LOE IIb). The results of the EPIC-Norfolk Study demonstrated that a higher intake of PUFA was not associated with the risk of type 2 diabetes mellitus [59] (LOE IIb). The Melbourne Collaborative Cohort was able to observe a positive, albeit not significant, association between intake of PUFA and risk of type 2 diabetes mellitus (RR highest quintile compared to lowest quintile $=1.29 ; 95 \%$ CI $0.84-1.97$ ) [62] (LOE IIb). An elevated risk with higher PUFA intake was also observed in the EPIC-Potsdam Study when coupled with a reduced intake of carbohydrates [63] (LOE IIb).

A higher intake of PUFA at the expense of SFA was associated with a significantly lower risk in the Iowa Women's Health Study [56] (LOE IIb) and the Nurses' Health Study [54] (LOE IIb), but not in the EPIC-Norfolk Study [59] (LOE IIb) and the EPIC-Potsdam Study [66] (LOE IIb) (also see chapter on SFA).

In the Atherosclerosis Risk In Communities Study, the proportion of PUFA in cholesteryl esters and phospholipids in plasma was not significantly associated with the risk of diabetes [67] (LOE IIb). In this study, 2,909 men and women were observed over a period of 9 years $(252$ new diseases). Likewise, in the Kuopio Ischemic Heart Disease Risk Factor Study, the proportion of PUFA in serum was not a significant predictor for the development of an impaired glucose tolerance or type 2 diabetes mellitus [58] (LOE IIb). In the Västerbotten Intervention Programme (159 new diseases) [68] (LOE IIb) and the EPICPotsdam Study (673 new diseases) [66] (LOE IIb), there was no association between the proportion of PUFA in erythrocyte membranes and the risk of diabetes.

There are numerous studies on the role of PUFA in the prevention of type 2 diabetes mellitus. However, their results are inconsistent. Therefore, the evidence that a higher intake of PUFA goes along with a reduced risk of type 2 diabetes mellitus is judged as insufficient. The evidence that the substitution of SFA with PUFA lowers the risk of type 2 diabetes mellitus is judged as insufficient due to the low number of studies and their heterogeneity.

\subsection{2 n-6 Fatty Acids}

Linoleic acid intake was not associated with risk of diabetes in the Health Professionals Follow-Up Study (RR for extreme quintiles $=0.89 ; 95 \%$ CI 0.74-1.06) [57] (LOE IIb) and the EPIC-Potsdam Study (RR for extreme quintiles $=1.11 ; 95 \%$ CI $0.79-1.56)$ [66] (LOE IIb). In the
Women's Health Study too, intake of n-6 fatty acids was not significantly associated with risk of diabetes $(\mathrm{RR}=0.95 ; 95 \%$ CI 0.78-1.16) [65] (LOE IIb). Likewise, in the Singapore Chinese Health Study, no association was observed with the intake of n-6 fatty acids (RR for extreme quintiles $=0.93,95 \%$ CI 0.87-1.12) [69] (LOE IIb). In the Melbourne Collaborative Cohort Study, a positive but not significant - association between intake of linoleic acid and risk of diabetes was observed (RR highest quintile compared to lowest quintile $=1.42 ; 95 \%$ CI 0.93 2.18) [62] (LOE IIb).

The prospective studies indicate with possible evidence that no association exists between intake of $n-6$ fatty acids (linoleic acid) and risk of type 2 diabetes mellitus.

\subsection{3 n-3 Fatty Acids}

The results of 7 prospective cohort studies $(7,365$ new diseases) on the association between ALA intake and risk of diabetes were summarised in a meta-analysis in 2012. This meta-analysis found an inverse but statistically insignificant association (RR per $0.5 \mathrm{~g} /$ day $=0.93$; 95\% CI 0.83-1.04) [70] (LOE IIa). There was marked heterogeneity between the studies. Higher concentrations of ALA in the blood were also associated with a reduced risk of diabetes, according to a meta-analysis of 6 cohort studies. However, in this case, too, the association did not reach statistical significance (RR per $0.1 \%$ higher proportion of total fatty acids $=0.90 ; 95 \% \mathrm{CI}$ 0.80-1.00) [70] (LOE IIa).

With possible evidence, a higher intake of ALA has no effect on the risk of diabetes.

Multiple meta-analyses have recently summarised the results of prospective cohort studies on long-chain n-3 fatty acids, with predominantly the same studies taken into account. The most comprehensive meta-analysis to date (16 prospective cohort studies, 440,873 people, 21,512 new diseases) showed that intake of long-chain n-3 fatty acids (EPA and DHA) has no influence on risk of diabetes. The RR per $250 \mathrm{mg} /$ day determined across all cohorts was 1.04 (95\% CI 0.97-1.10) [70] (LOE IIa). In another meta-analysis, the RR for the comparison of extreme quintiles of intake was 1.04 (95\% CI 0.92-1.18) [71] (LOE IIa). Eleven cohorts were considered. In both meta-analyses, however, there was significant heterogeneity between the studies. As such, a higher intake of long-chain n-3 fatty acids tended to be associated with an 
increased risk of diabetes in North American study populations, while inverse associations were observed in Asian study populations. This was also observed in the meta-analysis performed by Wallin et al. [72] (LOE IIa) with data from 13 cohort studies. In the meta-analysis, data were subsequently stratified by geographic region. The RR per $300 \mathrm{mg}$ /day of long-chain n-3 fatty acids was 1.17 (95\% CI 1.09-1.26) in North American study populations (6 cohorts), 0.98 (95\% CI 0.70-1.37) in European study populations (3 cohorts) and 0.90 (95\% CI $0.82-$ 0.98) in Asian and Australian study populations (4 cohorts). There was also heterogeneity within the geographic regions, particularly in Europe. Five out of 6 American cohort studies indicated a positive association between intake of long-chain n-3 fatty acids and risk of diabetes.

In cohort studies that did not observe the intake of fatty acids but recorded the fatty acid profile in the blood, a higher proportion of long-chain n-3 fatty acids was not associated with the risk of diabetes. A meta-analysis of 5 such studies showed an RR (per 3\% higher proportion of total fatty acids) of 0.94 (95\% CI 0.75-1.17) [70] (LOE IIa).

Studies on the role of long-chain $n$ - 3 fatty acids in prevention of type 2 diabetes mellitus are very inconsistent. Results from meta-analyses of multiple cohort studies suggest with possible evidence that a higher intake of these fatty acids is not associated with the risk.

\subsubsection{Ratio of n-6 to n-3 Fatty Acids}

The ratio of $\mathrm{n}-6$ fatty acids to $\mathrm{n}-3$ fatty acids was investigated for an association with the incidence of diabetes in only a few prospective studies. In the Nurses' Health Study, the ratio of n-6 to n-3 fatty acids was not associated with the risk of diabetes [54] (LOE IIb). Nor was there any association in the Singapore Chinese Health Study (RR for comparison of extreme quintiles $=0.98$; 95\% CI 0.85-1.14) [69] (LOE IIb). In the Health Professionals Follow-Up Study, the RR for the comparison of extreme quintiles of the ratio of n-3 to n- 6 fatty acids was 1.10 , with the association not reaching significance (95\% CI $0.92-1.31, \mathrm{p}$ for trend $=0.73$ ) [57] (LOE IIb). It was only in the Melbourne Collaborative Cohort Study that a higher ratio of n-6 to n-3 was associated with a higher risk ( RR for comparison of extreme quartiles $=1.56 ; 95 \% \mathrm{CI}$ 1.03-2.36) [62] (LOE IIb).

With possible evidence, the ratio of n-6 to n-3 fatty acids has no effect on the risk of type 2 diabetes mellitus.

\subsection{Trans Fatty Acids}

In the Iowa Women's Health Study, a negative relation was found between intake of trans fatty acids and incidence of diabetes after 11 years of observation (RR for extreme quintiles $=0.83 ; 95 \%$ CI $0.70-0.97)$ [56] (LOE IIb). In the Nurses' Health Study, a higher proportion of trans fatty acids of total energy intake correlated with an increased risk of diabetes. This association also remained after control for various confounding factors (RR for extreme quintiles $=1.31 ; 95 \%$ CI 1.10-1.56) [54] (LOE IIb). In the Health Professionals Follow-Up Study, on the other hand, no association between intake of trans fatty acids and risk of diabetes could be observed (RR for extreme quintiles $=0.90 ; 95 \%$ CI 0.74-1.10) [57] (LOE IIb). In the Women's Health Study, no significant association was observed between intake of trans fatty acids and risk of diabetes $(\mathrm{RR}$ for extreme quintiles $=1.03$; 95\% CI 0.83-1.28) when adjusted for BMI, energy intake, other confounders and total fat intake [65] (LOE IIb). The Melbourne Collaborative Cohort Study was also unable to identify any association [62] (LOE IIb).

In the Melbourne Collaborative Cohort Study, an inverse association was observed between trans fatty acid content in plasma phospholipids and risk of type 2 diabetes mellitus (RR for extreme quintiles of trans fatty acid content $=0.30 ; 95 \%$ CI 0.17-0.51) [62] (LOE IIb). A risk reduction was also described for trans-palmitoleic acid in the Cardiovascular Health Study (RR for extreme quintiles $=0.38 ; 95 \%$ CI 0.24-0.62) [73] (LOE IIb). Unlike these studies, in the EPIC-Potsdam Study associations between the relative proportion of trans fatty acids $(\mathrm{RR}=$ 0.88 ; $95 \%$ CI $0.63-1.24$ ) or the proportion of trans-palmitoleic acid in erythrocyte phospholipids $(\mathrm{RR}=0.89 ; 95 \%$ CI 0.64-1.23) and risk of diabetes following adjustment for risk factors for diabetes were markedly weaker and not statistically significant [66] (LOE IIb).

Due to the strongly different results from the cohort studies conducted to date, the evidence for an association between the intake of trans fatty acids and risk of type 2 diabetes mellitus is judged as insufficient.

\subsection{Need for Research - Type 2 Diabetes Mellitus}

The effect of PUFA on the risk of type 2 diabetes mellitus when compared to the effect of SFA has not been sufficiently documented thus far. Differences also appear to exist between different types of PUFA. In particular, 
the more recent results regarding a potential risk increase due to a higher intake of long-chain n-3 fatty acids in North American and, in some cases, in European cohorts should be analysed further. Future studies should more closely consider various types of fish as sources of longchain n-3 fatty acids as well as their preparation and content of heavy metals or persistent organic pollutants. The role of n-6 and n-3 fatty acids from plant-based foods (linoleic acid and ALA), particularly compared to SFA but also in ratio to one another, also remains unclear.

In the chapter on diabetes, SFA were not differentiated by their chain length or by whether they have an even or odd number of $\mathrm{C}$ atoms. The proportion of SFA with an odd number of $\mathrm{C}$ atoms in the blood is a biomarker for the intake of milk fat. Initial studies indicate that these SFA could differ from other SFA in their association with the risk of type 2 diabetes mellitus [e.g. 66, 74]. In that case, high-fat dairy products should reduce the risk of type 2 diabetes mellitus, but this is rather seen in low-fat products [75]. Future studies should differentiate between various SFA in the context of different food sources.

The question of whether trans fatty acids increase the risk of type 2 diabetes mellitus remains unanswered. Studies conducted thus far have provided contradictory results. Therefore, this question should be evaluated in additional cohort studies. As nutrient databases do not generally provide data on the trans fatty acid content of foods, the analysis of trans fatty acid content in the blood (cholesteryl esters or phospholipids) would be one alternative. Such analyses should differentiate between trans fatty acids as a product of industrial fat-hardening and those produced in the stomachs of ruminant animals.

\section{Quantity and Quality of Dietary Fat and Fatty Acid Intake and Primary Prevention of Dyslipoproteinaemia}

The aim of primary prevention of hereditary dyslipoproteinaemias is to delay and/or attenuate the manifestation of the disease. For healthy individuals, the aim is to avoid the occurrence of nutrition-related dyslipoproteinaemia.

Individual intervention studies or prospective cohort studies on the long-term primary prevention of primary dyslipoproteinaemia related to the intake of isolated nutritional factors such as total fat, SFA, MUFA, PUFA, n-3 fatty acids trans fatty acids or dietary cholesterol could not be found.

Evidence-Based Guideline of the German Nutrition Society
The individual observations are preceded by studies that involve the primary prevention of dyslipoproteinaemia in a multifactorial approach with changes of fat intake. These should make it clear that the influence of a change in fat intake on the plasma lipoprotein pattern is dependent on many additional factors, which can simultaneously be the direct or indirect subject of the intervention, for example body weight, energy or fibre intake.

Individual fatty acids can have different effects on the plasma concentrations of LDL and HDL cholesterol and also triglycerides. They are therefore dealt with separately in the following section. Total and LDL cholesterol generally react in the same way, sometimes with varying levels of significance. Their changes are therefore presented together. For patients with genetically determined primary dyslipoproteinaemias, the reactions of plasma lipoproteins may be stronger or weaker compared to healthy people. However, they generally go in the same direction.

In this 2nd version of the guideline, results from studies on secondary prevention were not included, as the focus of the German Nutrition Society's work is the nutrition of healthy people (primary prevention). Intervention studies and meta-analyses, which only deal with secondary prevention, were therefore not taken into account in the evaluation of evidence. In many meta-analyses, however, there was no separation between intervention studies on primary and secondary prevention. In such cases, the common effect estimate was specified. The inclusion of results from all meta-analyses from intervention studies on secondary prevention would in some cases provide a different evaluation of the evidence.

\subsection{Total Fat}

In an intervention study involving 1,182 school children, a significant decrease in the plasma concentration of total and HDL cholesterol was found in the intervention group when compared to the control group after reducing the proportion of total fat and SFA of energy intake over a period of 2 years [76] (LOE Ib).

Yu-Poth et al. [21] (LOE Ia) evaluated 37 nutritional intervention studies published between 1975 and 1981 in which total fat intake in the intervention groups was between $<10$ and 32 en\% (all interventions were either Step I or Step II diets from the National Cholesterol Education Program). In the evaluation, 11,586 healthy adults and at-risk patients of both sexes who were living indepen- 
dently were considered (intervention: 9,276 people, control: 2,310 people). The included intervention studies had very different designs and lasted between 3 weeks and 4 years. The evaluation showed that there was a significant positive relation between the proportion of total fat of energy intake and concentrations of total cholesterol, LDL cholesterol and HDL cholesterol. According to this meta-analysis, a 1-en\% reduction in total fat intake led to a decrease in concentrations of total cholesterol, LDL cholesterol and HDL cholesterol of $0.06 \mathrm{mmol} / \mathrm{l}$ (approx. $2.3 \mathrm{mg} / \mathrm{dl} ; 0.9 \%$ ), $0.042 \mathrm{mmol} / \mathrm{l}$ (approx. $1.6 \mathrm{mg} / \mathrm{dl} ; 1.0 \%$ ) and $0.01 \mathrm{mmol} / \mathrm{l}$ (approx. $0.39 \mathrm{mg} / \mathrm{dl} ; 0.8 \%$ ). The plasma triglyceride concentration remained unchanged, however.

These findings were supplemented by studies in which the intake of total fat and SFA was reduced simultaneously. In the randomised, double-blind, controlled 3 -stage crossover study of 86 healthy men aged from 22 to 64 presented by Lefevre et al. [77] (LOE Ib), in a relatively short period of only 6 weeks the effect of a reduced intake of total fat and SFA (30 en\% fat and 9 en\% SFA or 25 en $\%$ fat and 6 en\% SFA) was compared with an average American diet of 38 en $\%$ fat and 14 en\% SFA as the control diet. The intake levels of cholesterol, MUFA and PUFA were lower and the proportion of carbohydrates of energy intake was higher than in the control diet while the proportion of protein was constant. The reduction of total fat intake and SFA intake resulted in significant decreases in concentrations of total, LDL and HDL cholesterol and increased the ratio of total cholesterol to HDL cholesterol because of a smaller reduction in the concentration of LDL cholesterol compared to HDL cholesterol. There was also a significant increase in triglyceride concentration due to a higher intake of carbohydrates and a reduced intake of MUFA and PUFA.

The most recent and comprehensive investigation into the effect of a reduced or modified intake of fats on blood fats at the time this guideline was published is the meta-analysis carried out by Hooper et al. [25] (LOE Ia) in which a total of 48 intervention studies with more than 65,000 adults in total were evaluated. Studies involving people irrespective of their cardiovascular risk and existing CHD were included in the meta-analysis and it was not always possible to consider all studies for each parameter. Only studies with diseased patients, pregnant or nursing women, studies that did not meet the authors' criteria and studies with missing information were excluded. In the context of the meta-analysis by Hooper et al. [25] (LOE Ia), the reduction of fat intake was defined as a reduced en\% proportion of fats with comparable to- tal energy intake; a lower-fat diet existed, according to the authors, if fat intake was $<30 \mathrm{en} \%$ and the reduced proportion of fat was compensated by energy from carbohydrates and protein in the form of fruit and vegetables, for example. Studies involving supplements of ALA, n-3 fatty acids or fish oil, or a reduction in fat intake achieved using fat substitutes (e.g. olestra) were among other studies that were excluded from the meta-analysis. In the meta-analysis carried out by Hooper et al. [25] (LOE Ia), the effects of a reduced fat intake and a change in the composition of the consumed fats on the concentrations of total, LDL and HDL cholesterol and triglycerides in comparison with a regular diet were studied as tertiary endpoints. All of the measures lowered total cholesterol concentration. However, in contrast to the studies published before the meta-analysis by Hooper et al. [25] (LOE Ia), no effect on the concentration of HDL cholesterol could be determined. A reduction in total fat intake led to a significant reduction in the concentration of LDL cholesterol; yet, in these studies carried out over a longer period it had no effect on triglyceride concentration.

The evidence is convincing that a reduction in the proportion of fat of energy intake and therefore total fat intake in the context of a diet with current fat content and current fat quality lowers the concentration of total and $L D L$ cholesterol in plasma.

There is possible evidence for a reduction in the plasma concentration of HDL cholesterol due to a reduction in the proportion of fat of energy intake.

There is probable evidence that a reduction in the proportion of fat of energy intake has no long-term effect on the plasma triglyceride concentration.

The evidence for a reduction in the ratio of total cholesterol to HDL cholesterol and LDL to HDL cholesterol through a reduction in the proportion of fat of energy intake is insufficient.

\subsection{SFA}

SFA and Concentration of Total and LDL Cholesterol

Compared to carbohydrates, long-chain SFA (C12:0, C14:0, C16:0) raised total and LDL cholesterol concentrations by around twice as much as the same amount of PUFA (C18:2 n-6) lowered total and LDL cholesterol concentration ([78] LOE Ib, [21, 79, 80] LOE Ia). Myristic (C14:0) and palmitic acid (C16:0) increased LDL cholesterol concentration the most, according to a meta-analy- 
sis of 60 studies with 1,672 adults carried out by Mensink et al. [80] (LOE Ia). Long-chain saturated stearic acid (C18:0) changed total cholesterol in plasma compared to carbohydrates just as little as saturated MCT [81, 82] (LOE Ib). In the meta-analysis of 224 studies involving 8,143 adults published by Howell et al. [83] (LOE Ia), a 1 -en $\%$ reduction in the proportion of SFA in the diet led to a reduction in the LDL cholesterol concentration of 1.8 $\mathrm{mg} / \mathrm{dl}(=0.047 \mathrm{mmol} / \mathrm{l})$. The meta-analysis carried out by Yu-Poth et al. [21] (LOE Ia) showed that a 1-en\% reduction in the proportion of dietary SFA led to a reduction in total cholesterol concentration of $0.056 \mathrm{mmol} / \mathrm{l}(=2.2 \mathrm{mg} /$ $\mathrm{dl})$ and a reduction in LDL cholesterol concentration of $0.05 \mathrm{mmol} / \mathrm{l}(=1.9 \mathrm{mg} / \mathrm{dl})$.

The most comprehensive and recent study to date regarding the effect of a modified intake of fats on blood fats is the meta-analysis carried out by Hooper et al. [25] (LOE Ia) as presented in section 5.1. As part of this metaanalysis, a modification of fat intake was defined as a proportion of total fats of $\geq 30 \mathrm{en} \%$ and therefore, a virtually unchanged total fat intake compared to corresponding control groups and also a higher intake of MUFA and PUFA than in a 'normal' diet. A modification of the composition of the ingested fats significantly reduced total cholesterol concentration. However, in this meta-analysis it could not be determined whether the modification has a significant effect on the LDL cholesterol concentration, as this parameter has been measured only in 2 individual studies [84, 85].

Once a year, Niinikoski et al. [86] (LOE Ib) carried out nutritional consultations, starting at the age of 7 months up until the age of 14, with 540 children and their parents. The control group with 522 children received no consultations. In the consultations, the importance of a diet with reduced SFA and cholesterol content and a reduction in total fat intake to between 30 and 35 en\% was communicated. The consultations led to a reduced intake of total fat and SFA. The concentrations of total and LDL cholesterol were significantly lower up to the age of 14 . In the continuation of the study by Niinikoski et al. [86] (LOE $\mathrm{Ib}$ ) up to the age of 19 [87] (LOE Ib), the annual nutritional consultation led to a reduced intake of SFA; the difference in total fat intake between the intervention group and the control group decreased with age until in 17-year-old girls and 19-year-old boys there were no longer any differences. The lower intake of SFA was accompanied by significantly lower LDL cholesterol concentrations in both groups. In boys, total cholesterol concentration was reduced significantly with a lower intake of SFA; this was not the case for girls.

Evidence-Based Guideline of the German Nutrition Society
In the intervention study by Müller et al. [88] (LOE Ib) involving 25 healthy women with an average age of $30.5 \pm 9.8$, a low-SFA diet as opposed to a high-SFA diet did not lead to any significant changes in total and LDL cholesterol concentration.

\section{SFA and HDL Cholesterol Concentration}

Compared to unsaturated fatty acids or carbohydrates, long-chain SFA increased the HDL cholesterol concentration $[79,80]$ (LOE Ia); lauric acid has the most significant effect here [80] (LOE Ia). In the study published by Müller et al. [88] (LOE Ib), a reduced intake of SFA led to a significant reduction in HDL cholesterol concentration. In the study by Niinikoski et al. [87] (LOE Ib), a reduced intake of SFA did not result in any changes in HDL cholesterol concentration in young people. In contrast to earlier studies, the meta-analysis carried out by Hooper et al. [25] (LOE Ia) could not identify any effect on HDL cholesterol concentration caused by the modification of the ingested fats.

\section{SFA and Triglyceride Concentration}

The increased intake of long-chain SFA lowered the triglyceride concentration in fasting plasma $[79,80,83]$ (LOE Ia). Müller et al. [88] (LOE Ib) demonstrated that a reduced intake of SFA raised the plasma triglyceride concentration. In a further meta-analysis with studies carried out over longer periods, however, there was no significant relation between SFA intake and plasma triglyceride concentration [21] (LOE Ia). In the study carried out by $\mathrm{Ni}$ inikoski et al. [87] (LOE Ib), boys had significantly lower triglyceride concentrations when SFA intake was lower; yet this was not the case for girls. In contrast to a reduction in fat intake, a modification of the ingested fats led to a reduction in the triglyceride concentration [25] (LOE Ia).

\section{SFA and the Ratio of Total to HDL Cholesterol}

In the meta-analysis performed by Mensink et al. [80] (LOE Ia), SFA did not lead to a change in the ratio of total to HDL cholesterol when compared with carbohydrates, as in this study there was an increase in the concentration of both total and HDL cholesterol due to the increased intake of SFA.

\section{SFA and the Ratio of LDL to HDL Cholesterol}

In the intervention study by Müller et al. [88] (LOE Ib), a reduced intake of SFA led to an increase in the ratio of LDL to HDL cholesterol due to the significant decrease in the HDL cholesterol concentration. 
The evidence is convincing that intake of dietary SFA increases the plasma concentrations of total and LDL cholesterol, while a reduction in SFA intake (except for stearic acid and MCT) reduces them.

There is possible evidence that an increased intake of SFA increases the plasma concentration of HDL cholesterol.

The evidence for a reduction in plasma triglyceride concentration after increasing intake of SFA for a short period of time is convincing.

There is probable evidence that SFA intake has no effect on the ratio of total to HDL cholesterol.

The evidence for an association between SFA intake and the ratio of LDL to HDL cholesterol is insufficient.

\subsection{MUFA}

MUFA and Total and LDL Cholesterol Concentration According to Mensink et al. [80] (LOE Ia) MUFA (18:1) significantly lowered the concentration of LDL cholesterol when compared with carbohydrates; the slight concentration-lowering effect on total cholesterol was not significant, however. In a randomised, parallel group, controlled intervention study, Bos et al. [89] (LOE Ib) compared a high-MUFA diet and a Mediterranean diet with a high-SFA diet in 57 non-diabetics aged between 40 and 65 with a BMI $\geq 25$ or a hip measurement of $\geq 94 \mathrm{~cm}$ in men and $\geq 80 \mathrm{~cm}$ in women over the course of 8 weeks following a 2-week high-SFA run-in-phase. In both diets, a significant reduction in total and LDL cholesterol concentration was observed when compared with a high-SFA diet, although there were no differences between the high-MUFA diet and the Mediterranean diet. In a randomised, crossover intervention study of 15 adults (5 men and 10 women) aged between 36 and 69, AllmanFarinelli et al. [90] (LOE Ib) studied the effect of a highMUFA diet (high-oleic sunflower oil; 32.6 en\% total fat, 8.8 en\% SFA, 3.5 en\% PUFA and 20.3 en\% MUFA) compared to a high-SFA diet (33.1 en\% total fat, 20.8 en\% SFA, 2.7 en\% PUFA, 9.6 en\% MUFA). The high-MUFA intervention led to a significant decrease in total and LDL cholesterol concentration in comparison with the highSFA diet. An additional meta-analysis showed that the substitution of SFA with MUFA led to a decrease in total and LDL cholesterol concentration; no significant difference could be determined between the effect of MUFA and PUFA [91] (LOE Ia). In this meta-analysis, 14 studies involving a total of 273 men and 166 women aged be- tween 18 and 78 with an intervention period of between 21 and 84 days were considered. In the individual studies, various high-MUFA oils (olive oil, high-oleic safflower oil, rapeseed oil or different mixtures of olive and rapeseed oil, olive and peanut oil and olive and sunflower oil) were compared with various high-PUFA oils (grape seed oil, safflower oil, corn oil, sunflower oil or a mixture of safflower and corn oil).

While SFA raise total and LDL cholesterol concentration, MUFA lower total and LDL cholesterol concentration slightly compared to carbohydrates, according to the meta-analysis of 27 studies involving 682 adults carried out by Mensink and Katan [79] (LOE Ia). However, the effect is not significant. Likewise, in the metaanalysis of 12 intervention studies with a minimum duration of 6 months and a maximum of 4 years involving a total of 1,990 individuals carried out by Schwingshackl et al. [37] (LOE Ia), no significant difference could be determined between a high $(>12$ en $\%)$ and low $(\leq 12$ en\%) intake of MUFA regarding the total and LDL cholesterol concentration. In the analysed studies, highMUFA diet types were compared with various lowMUFA diet types (low fat content, low or high glycaemic index, high-PUFA, high-protein or a control diet type that does not fit into these categories with a proportion of total fat $\geq 30 \mathrm{en} \%$ and/or a proportion of SFA $\geq 10$ en\%). The study by Wien et al. [92] in which the effects of increased MUFA intake in form of increased almond consumption were examined as part of the intervention was also one of the studies analysed. However, fats from almonds are only partially resorbed [93]. Furthermore, the study by Shai et al. [94] involving patients with CHD was included in the overall result. The authors specify, however, that a re-analysis without these 2 studies by Wien et al. [92] and Shai et al. [94] provides the same overall result. The results of the metaanalysis are also to be treated with caution due to the inclusion of studies with differing weight effects and types of diet. Thijssen and Mensink [95] (LOE Ib) compared the effect of a 5-week diet in 19 men and 27 women aged between 18 and 65 . The diet provided 38 en $\%$ fat - around 7 en \% were in the form of stearic, oleic or linoleic acid. In this study too, no significant changes in total and LDL cholesterol concentration could be observed in any of the 3 diets.

\section{MUFA and HDL Cholesterol Concentration}

According to Mensink and Katan [96] (LOE Ib), a diet high in fat and MUFA with olive oil as basis prevented a decrease in HDL cholesterol concentration compared to 
a low-fat diet with a high proportion of complex carbohydrates and dietary fibres. The meta-analysis carried out by Mensink and Katan [79] (LOE Ia) showed that substituting carbohydrates with MUFA leads to an increase in HDL cholesterol concentration.

In the meta-analysis by Gardner and Kraemer [91] (LOE Ia), substitution of SFA with MUFA did not cause a change in HDL cholesterol concentration. In the intervention studies of Allman-Farinelli et al. [90] (LOE Ib), Bos et al. [89] (LOE Ib) and Thijssen and Mensink [95] (LOE Ib), there was also no indication that a high-MUFA diet changes the concentration of HDL cholesterol when compared to a high-SFA diet.

\section{MUFA and Triglyceride Concentration}

According to the meta-analyses by Mensink and Katan [79] (LOE Ia) and Mensink et al. [80] (LOE Ia), MUFA lowered the plasma triglyceride concentration when compared to carbohydrates. The intervention studies carried out by Allman-Farinelli et al. [90] (LOE Ib) and Bos et al. [89] (LOE Ib) also showed a decrease in triglyceride concentration for a high-MUFA intervention compared to a high-SFA diet.

In the meta-analysis performed by Gardner and Kraemer [91] (LOE Ia), MUFA raised the plasma triglyceride concentration by $0.14 \mathrm{mmol} / \mathrm{l}$ compared to PUFA; compared to SFA, however, MUFA had no effect. This was also the case in the intervention study of Thijssen and Mensink [95] (LOE Ib).

\section{MUFA and the Ratio of Total to HDL Cholesterol}

In the meta-analysis by Mensink et al. [80] (LOE Ia), MUFA lowered the ratio of total to HDL cholesterol compared to carbohydrates. Thijssen and Mensink [95] (LOE Ib) observed no changes with a high-MUFA diet compared to a high-SFA diet.

\section{MUFA and the Ratio of LDL to HDL Cholesterol}

Mensink and Katan [79] (LOE Ia) found a slight decrease in the ratio of LDL to HDL cholesterol when carbohydrates were replaced by MUFA.

The evidence for a lack of association between MUFA intake and the plasma concentrations of total and LDL cholesterol compared to carbohydrates is probable, and there is possible evidence that MUFA have a lowering effect on the plasma concentrations of total and LDL cholesterol when compared with longchain SFA.
The evidence is convincing that MUFA prevent a decrease in the plasma concentration of HDL cholesterol when compared with carbohydrates, and there is probable evidence for a lack of an association between intake of MUFA and plasma concentration of HDL cholesterol in comparison with long-chain SFA.

The evidence is convincing that MUFA lower the plasma triglyceride concentration when compared to carbohydrates, and there is possible evidence for a lack of an association between MUFA intake and plasma triglyceride concentration when compared with long-chain SFA.

The evidence that MUFA lower the ratio of total to HDL cholesterol and LDL to HDL cholesterol when compared to carbohydrates is convincing, and there is insufficient evidence for an association between MUFA intake compared to long-chain SFA and the ratio of total to HDL cholesterol and (due to a lack of studies) LDL to HDL cholesterol.

\subsection{PUFA}

\subsubsection{Total PUFA}

The meta-analysis by Gardner and Kraemer [91] (LOE Ia) showed that the substitution of SFA with PUFA lowers the total and LDL cholesterol concentration and has no effect on plasma HDL cholesterol and triglyceride concentration.

The evidence is convincing that the substitution of SFA with PUFA lowers plasma concentrations of total and LDL cholesterol.

There is probable evidence that the substitution of SFA with PUFA does not affect plasma concentrations of $H D L$ cholesterol and triglycerides.

There is (due to a lack of studies) insufficient evidence that replacing SFA with PUFA has an effect on the ratios of total to HDL cholesterol and LDL to HDL cholesterol.

\subsection{2 n-6 Fatty Acids}

Polyunsaturated n-6 Fatty Acids and Total and LDL Cholesterol Concentration

n-6 fatty acids (C18:2) lowered the concentrations of both total and LDL cholesterol around half as much as the same amount of SFA raised them [79, 80] (LOE Ia). An increase in the proportion of $n-6$ fatty acids in the diet of $1 \mathrm{en} \%$ with constant energy intake led to a reduction in total cholesterol concentration of $0.90 \mathrm{mg} / \mathrm{dl} \quad(=23.3$ $\mu \mathrm{mol} / \mathrm{l})$ and a reduction in LDL cholesterol concentration of $0.5 \mathrm{mg} / \mathrm{dl}(=12.93 \mu \mathrm{mol} / \mathrm{l})$ [83] (LOE Ia). 
Polyunsaturated n-6 Fatty Acids and HDL Cholesterol Concentration

According to the meta-analysis by Mensink and Katan [79] (LOE Ia) and Mensink et al. [80] (LOE Ia), the substitution of SFA with n-6 fatty acids led to a small reduction in the concentration of HDL cholesterol, whereas there was a significantly greater reduction when SFA were replaced by carbohydrates. The extent of this reduction is, according to the study of Mensink et al. [80] (LOE Ia), dependent on the type of SFA; as such, the effect decreased from C12:0 to C18:0.

\section{Polyunsaturated n-6 Fatty Acids and Triglyceride}

Concentration

Compared to carbohydrates, n-6 fatty acids lowered the triglyceride concentration at a rate comparable to SFA and MUFA $[79,80]$ (LOE Ia).

\section{Polyunsaturated n-6 Fatty Acids and the Ratio of \\ Total to HDL Cholesterol}

The ratio of total to HDL cholesterol fell the most when trans fatty acids and SFA were replaced by $n-6$ fatty acids [80] (LOE Ia). The substitution of carbohydrates or SFA with n-6 fatty acids lowered the ratio of total to HDL cholesterol on a similar level to the substitution with MUFA $[79,80]$ (LOE Ia).

Polyunsaturated n-6 Fatty Acids and the Ratio of LDL to HDL Cholesterol

No studies investigating the effect of n- 6 fatty acid intake on the ratio of LDL to HDL cholesterol could be found.

There is convincing evidence that an increase in the proportion of n- 6 fatty acids in the diet lowers plasma concentrations of total and LDL cholesterol.

There is convincing evidence that an increase in the proportion of $n-6$ fatty acids in the diet reduces the plasma HDL cholesterol concentration.

The evidence that an increase in the proportion of n-6 fatty acids in the diet at the expense of carbohydrates lowers the plasma triglyceride concentration is convincing, while there is probable evidence that an increase in $n-6$ fatty acids by replacing other fatty acids has no effect on the plasma triglyceride concentration.

There is convincing evidence that $n-6$ fatty acids reduce the ratio of total to HDL cholesterol, whereas (due to the lack of studies) the evidence for an influence on the ratio of LDL to HDL cholesterol is insufficient.

\subsection{3 n-3 Fatty Acids}

\section{ALA}

ALA and Total and LDL Cholesterol Concentration

In the 6-week, parallel, blind, randomised and controlled intervention study on 74 healthy persons (27 men and 47 women) aged 19-43 presented by Egert et al. [97] (LOE Ib), no influence of a daily intake of $4.4 \mathrm{~g}$ ALA on total cholesterol concentration was shown. After consuming foods enriched with ALA, a significant reduction of total [98] (LOE Ib) and LDL cholesterol concentration $[97,98]$ (LOE Ib) was achieved after an intervention of 4 and 6 weeks, respectively. In the 2-week study by Sanders and Roshanai [99] (LOE Ib) on 3 men and 2 women, a daily intake of $9.38 \mathrm{~g}$ of ALA in the form of $20 \mathrm{ml}$ linseed oil had no influence on total cholesterol concentration in plasma. However, an effect of ALA on total cholesterol concentration is not to be expected in such a short intervention. For this reason, this study was not taken into consideration for the evidence judgement for total cholesterol. An intervention study over 6 weeks on 29 men aged 18-35 showed that the comparison of an ALA-rich diet (10 g ALA and $12 \mathrm{~g} \mathrm{LA}, 15$ test persons) with an LArich diet (1 g ALA and $21 \mathrm{~g}$ LA, 14 test persons) did not result in any significant differences in total and LDL cholesterol concentration after 6 weeks [100] (LOE Ib). Another intervention study on 30 test persons over 6 months showed no significant differences in the isocaloric comparison of ALA (9.5 g per day) and LA in the form of specially produced margarine with regard to the lowering influence on total and LDL cholesterol concentration [101] (LOE Ib).

\section{ALA and HDL Cholesterol Concentration}

According to the intervention studies conducted by Egert et al. [97] (LOE Ib), Sanders and Roshanai [99] (LOE Ib) and Finnegan et al. [101] (LOE Ib), the HDL cholesterol concentration was not influenced by the daily intake of 4.4, 9.38 or $9.5 \mathrm{~g}$ ALA. In another study, the consumption of foods enriched with ALA led to a moderate increase in the concentration of HDL cholesterol [98] (LOE Ib).

\section{ALA and Triglyceride Concentration}

With an intake of very high quantities of ALA (38 g per day) compared to high quantities of LA ( $45 \mathrm{~g}$ per day), the triglyceride concentration in plasma was significantly lowered in the study by Singer et al. [102] (LOE Ib). In the intervention study on healthy men and women conducted by Egert et al. [97] (LOE Ib), the daily intake of $4.4 \mathrm{~g}$ ALA and the consumption of $2.2 \mathrm{~g}$ EPA or $2.3 \mathrm{~g}$ DHA per 
day also caused a significant lowering of the triglyceride concentration.

In the study by Sanders and Roshanai [99] (LOE Ib), on the other hand, a high daily intake of $9.38 \mathrm{~g}$ ALA in the form of $20 \mathrm{ml}$ linseed oil in contrast to $20 \mathrm{ml}$ fish oil, which equated to an intake of $3.03 \mathrm{~g}$ EPA and $2.93 \mathrm{~g}$ DHA per day, had no influence on the triglyceride concentration in plasma. In the study by Pang et al. [100] (LOE Ib), ALA (diet with $12 \mathrm{~g}$ LA and $10 \mathrm{~g}$ ALA per day) compared to LA (diet with $21 \mathrm{~g}$ LA and $1 \mathrm{~g}$ ALA per day) with isocaloric nutrition did not lower the triglyceride concentration in the plasma either. The study by Karvonen et al. [98] (LOE Ib) showed comparable results.

In the intervention study conducted by Finnegan et al. [101] (LOE Ib), however, an intake of $9.5 \mathrm{~g}$ ALA per day led to a significant increase $(+10.9 \%)$ in the triglyceride concentration in plasma compared to fish oil (with a total amount of EPA and DHA of $1.7 \mathrm{~g}$ per day).

\section{ALA and the Ratio of Total to HDL Cholesterol}

After a daily intake of $6.3 \mathrm{~g}$ ALA compared to the same amount of LA, the ratio of total to HDL cholesterol increased [103] (LOE Ib). In the intervention study presented by Egert et al. [97] (LOE Ib), there was no significant reduction in the ratio of total to HDL cholesterol through the intake of $4.4 \mathrm{~g}$ ALA per day.

\section{ALA and the Ratio of LDL to HDL Cholesterol}

No study could be identified for the research period in which an influence of ALA on the ratio of LDL to HDL cholesterol was examined.

The evidence is probable that ALA lowers the total cholesterol concentration in plasma.

The evidence is convincing that ALA lowers the $L D L$ cholesterol concentration in plasma.

The evidence for a lack of influence of ALA on the HDL cholesterol concentration in plasma is probable.

There is insufficient evidence for an influence of $A L A$ on the triglyceride concentration in plasma.

The evidence is insufficient that ALA influences the ratio of total to $H D L$ cholesterol and of $L D L$ to $H D L$ cholesterol (not enough studies available).

\section{Long-Chain n-3 Fatty Acids}

Long-Chain n-3 Fatty Acids and Total and LDL

Cholesterol Concentration

In the study by Schaefer et al. [104] (LOE Ib), the effect of 2 National Cholesterol Education Program (NCEP) Step II diets with $\leq 30 \mathrm{en} \%$ total fat, $<7$ en $\%$ SFA and $<200$ mg cholesterol per day on total and LDL cholesterol concentrations was examined on 22 men and women with a mean age of $63 \pm 10$ years. Due to different frequencies in the consumption of fish, the diets either had a high or a low level of $n-3$ fatty acids $(0.1 \pm 0.1$ en\% as eicosatetraenoic acid, $0.2 \pm 0.1 \mathrm{en} \%$ as EPA and $0.5 \pm 0.2 \mathrm{en} \%$ as DHA vs. $<0.02 \mathrm{en} \%$ as eicosatetraenoic acid, $<0.02 \mathrm{en} \%$ as EPA and $0.1 \pm 0.1 \mathrm{en} \%$ as DHA). The higher intake of longchain n-3 fatty acids associated with the higher intake of fish led to a significant reduction in total and LDL cholesterol concentration, with the influence of the elimination of SFA also presumably having an effect.

In a randomised, double-blind, placebo-controlled crossover study, Watanabe et al. [105] (LOE Ib) examined the influence of the intake of $1.26 \mathrm{~g}$ EPA and $0.54 \mathrm{~g}$ DHA per day in form of fish oil capsules over a 4 -week period on 17 Japanese males who consumed relatively high amounts of fish. The intake of fish oil capsules did not cause any changes in total and LDL cholesterol concentration. It is not clear if the reason why there was no effect was due to the relatively high fish consumption of the study participants. In the randomised intervention study conducted by García-Alonso et al. [106] (LOE Ib) on 18 healthy women aged 35-55, the daily intake of tomato juice enriched with $250 \mathrm{mg}$ EPA and $181 \mathrm{mg}$ DHA did not lead to any changes in total and LDL cholesterol concentration either. However, the daily amount of n-3 fatty acids ingested in this study is very low, so that an effect is perhaps not to be expected. Cazzola et al. [107] (LOE Ib) examined a possible dosage and age dependence of the consumption of EPArich oil in a 12-week randomised intervention study on 93 healthy male non-vegetarians who did not consume any fish. The intake of $1.35,2.70$, or $4.05 \mathrm{~g}$ EPA per day had no influence on the concentration of total or LDL cholesterol in the 18-42 age group or in the group aged from 53 to 70. The effect of a daily intake of $3.1 \mathrm{~g} \mathrm{n}-3$ fatty acids ( $1.8 \mathrm{~g}$ EPA, $0.2 \mathrm{~g}$ DPA and $1.1 \mathrm{~g} \mathrm{DHA}$ ) in comparison to the daily intake of $3.7 \mathrm{~g}$ oleic acid was examined in the 8-week randomised study by Damsgaard et al. [108] (LOE Ib) on 64 healthy men aged from 19 to 40 . In addition to this, each of the 2 groups was further subdivided into 2 subgroups, one with a high and one with a low intake of ALA. No influence of n-3 fatty acids on total and LDL cholesterol concentration was found here either. In a double-blind crossover intervention study conducted by Fontani et al. [109] (LOE Ib) on 33 healthy persons (13 men and 20 women) aged 22-51 over 70 days, there was also no change in total cholesterol concentration with an intake of $4 \mathrm{~g}$ fish oil capsules (1.6 $\mathrm{g}$ EPA and $0.8 \mathrm{~g}$ DHA plus $0.4 \mathrm{~g}$ of additional $\mathrm{n}-3$ fatty acids) per day compared to the intake of $4 \mathrm{~g}$ olive oil 
capsules per day. Similarly, no influence of $2.2 \mathrm{~g}$ EPA, $2.3 \mathrm{~g}$ DHA and $4.4 \mathrm{~g}$ ALA per day over 6 weeks on the total and LDL cholesterol concentration was found in the 6-week parallel, blind, randomised and controlled intervention study on 74 healthy persons ( 27 men and 47 women) aged 19-43 presented by Egert et al. [97] (LOE Ib). The fatty acids were supplied in the form of roughly $30 \mathrm{~g}$ of margarine per day. Kaul et al. [110] (LOE Ib) examined the influence of $2 \mathrm{~g}$ of various oils administered daily in capsule form in a double-blind, placebo-controlled intervention study over 12 weeks on 34 healthy men and 54 healthy women. The intake of n-3 fatty acids was $606 \mathrm{mg}$ per day in the group treated with fish oil, 1,022 $\mathrm{mg}$ in the linseed oil group, $372 \mathrm{mg}$ in the group treated with hemp oil and $30 \mathrm{mg}$ in the control group (sunflower oil). The daily intake of n- 6 fatty acids was 1,374 $\mathrm{mg}$ (control), $30 \mathrm{mg}$ (fish oil), $298 \mathrm{mg}$ (linseed oil) and 1,196 mg (hemp oil). None of the interventions led to a significant change in total and LDL cholesterol concentration.

However, increases in total and LDL cholesterol concentration through n-3 fatty acids were observed in other studies. 485 healthy men and women were included in a meta-analysis of 11 randomised, controlled studies from the years 1996 to 2011 [111] (LOE Ia) in order to examine the influence of the intake of DHA-rich algae oil on cardiovascular risk factors. The mean daily intake of DHA was $1.68 \mathrm{~g}$ and led to a significant average increase in the concentration of LDL cholesterol of $0.23 \mathrm{mmol} / \mathrm{l}$. The influence on the total cholesterol concentration was not evaluated in this study.

In the randomised, double-blind, placebo-controlled intervention study with parallel design conducted by Milte et al. [112] (LOE Ib) on 36 men and 31 women with an average age of 53 years, no influence on total cholesterol concentration was seen with a daily intake of 2,4 and $6 \mathrm{~g}$ of fish oil. However, a daily intake of $4 \mathrm{~g}$ of fish oil led to a significant increase in the concentration of LDL cholesterol of $10 \%$, which was not observed with daily intake levels of 2 and $6 \mathrm{~g}$. In the randomised, double-blind, placebo-controlled FINGEN Crossover Study conducted by Caslake et al. [113] (LOE Ib) on 312 men and women aged 20-70, the influence of a daily intake of 0.7 and $1.8 \mathrm{~g}$ of an oil containing EPA and DHA (with a ratio of DHA to EPA of $1: 1.5$ ) on total and LDL cholesterol concentration was examined in comparison to the same amount of a control oil consisting of $80 \%$ palm oil and $20 \%$ soya oil. No significant influence of the n-3 fatty acids on the total cholesterol concentration was observed in this study, but there was a significant increase in the LDL cholesterol concentration.

\section{Long-Chain n-3 Fatty Acids and HDL Cholesterol Concentration}

No indications of a change in HDL cholesterol concentration were found in several studies: on 67 healthy men and women with a mean age of $53 \pm 2$ years with an intake of 2, 4 or $6 \mathrm{~g}$ of fish oil per day [112] (LOE Ib), on 17 healthy Japanese males aged from 35 to 64 with high fish consumption and an additional intake of $1.7 \mathrm{~g}$ EPA and DHA per day [105] (LOE Ib), on 18 healthy women aged 35-55 with $250 \mathrm{mg}$ EPA and $181 \mathrm{mg}$ DHA as enriched tomato juice [106] (LOE Ib), on 93 men aged from 18 to 42 and 62 men aged from 53 to 70 with up to $4.05 \mathrm{~g}$ EPA per day [107] (LOE Ib), on 64 healthy men aged from 19 to 40 with $3.1 \mathrm{~g} \mathrm{n}-3$ fatty acids per day [108] (LOE Ib) and on 88 healthy men and women aged on average between 32.9 and 35.0 with $2 \mathrm{~g}$ of EPA and DHA-rich fish oil per day [110] (LOE Ib).

Other studies showed that an intervention with n-3 fatty acids resulted in a significant increase in the HDL cholesterol concentration. In the meta-analysis conducted by Bernstein et al. [111] (LOE Ia), there was a mean significant increase in the HDL cholesterol concentration of $0.07 \mathrm{mmol} / \mathrm{l}$ with an average daily intake of $1.68 \mathrm{~g} \mathrm{DHA}$ as algae oil. Caslake et al. [113] (LOE Ib) found a significant increase in the HDL cholesterol concentration in the FINGEN Study conducted on 312 men and women aged from 20 to 70 with a daily intake of 0.7 or $1.8 \mathrm{~g}$ EPA and DHA. In the study conducted by Egert et al. [97] (LOE Ib) on 74 healthy men and women aged 19-43 years, there was no increase in the concentration of HDL cholesterol with a daily intake of $4.4 \mathrm{~g}$ ALA and $2.2 \mathrm{~g}$ EPA, but a daily intake of $2.3 \mathrm{~g}$ DHA caused a significant increase in the HDL cholesterol concentration.

\section{Long-Chain n-3 Fatty Acids and Triglyceride Concentration}

The meta-analysis conducted by Bernstein et al. [111] (LOE Ia) showed a significant mean reduction of the triglyceride concentration by $0.20 \mathrm{mmol} / \mathrm{l}$ with a mean daily intake of $1.68 \mathrm{~g} \mathrm{DHA}$ in the form of algae oil. Significant reductions of the triglyceride concentrations through intake of long-chain n-3 fatty acids also resulted in the studies by Milte et al. [112] (LOE Ib) with a daily intake of 4 and $6 \mathrm{~g}$ of fish oil, Cazzola et al. [107] (LOE Ib) on healthy men of various age groups with a daily intake of $4.05 \mathrm{~g}$ EPA, Caslake et al. [113] (LOE Ib; FINGEN Study) with a daily intake of 0.7 and $1.8 \mathrm{~g}$ EPA/DHA, Damsgaard et al. [108] (LOE Ib) with a daily intake of $3.1 \mathrm{~g}$ of n-3 fatty acids and Egert et al. [97] (LOE Ib) on healthy women and men with a daily consumption of $4.4 \mathrm{~g}$ ALA as well as 
$2.2 \mathrm{~g}$ EPA and $2.3 \mathrm{~g}$ DHA. In the study by Caslake et al. [113] (LOE Ib), the effect was greater among men than among women. In addition to this, the study by Cazzola et al. [107] (LOE Ib) showed that the reduction of the triglyceride concentration with a daily intake of $1.35 \mathrm{~g} \mathrm{EPA}$ was greater than with higher daily intake levels. In an intervention study by Finnegan et al. [101] (LOE Ib), EPA and DHA in a dose of $1.7 \mathrm{~g}$ per day caused a significant reduction in the triglyceride concentration in 39 healthy test persons over a period of 6 months.

Within the research period of this guideline, however, several studies also produced other results. Accordingly, there were no significant changes in the triglyceride concentrations in plasma in the studies by Kaul et al. [110] (LOE Ib) on 88 healthy men and women with an average age of between 32.9 and 35.0 years with $2 \mathrm{~g} n-3$ fatty acids per day, by García-Alonso et al. [106] (LOE Ib) on 18 healthy women aged from 35 to 55 with $250 \mathrm{mg}$ EPA and $181 \mathrm{mg}$ DHA in the form of enriched tomato juice per day, and by Fontani et al. [109] (LOE Ib) on 13 men and 20 women aged 22-51 with $4 \mathrm{~g}$ fish oil per day compared to $4 \mathrm{~g}$ olive oil.

\section{Long-Chain n-3 Fatty Acids and the Ratio of Total to} HDL Cholesterol

The randomised, placebo-controlled intervention study presented by Kaul et al. [110] (LOE Ib) with the administration of oil capsules rich in $\mathrm{n}-3$ fatty acids ( $2 \mathrm{~g}$ fish oil, $2 \mathrm{~g}$ linseed oil or $2 \mathrm{~g}$ hemp seed oil per day) compared to sunflower oil ( $2 \mathrm{~g}$ per day) as a control did not lead to any changes in the ratio of total to HDL cholesterol. In the intervention study by Egert et al. [97] (LOE Ib), the intake of ALA or EPA did not produce any changes in the ratio of total to HDL cholesterol, whereas an increased intake of DHA significantly lowered the ratio of total to HDL cholesterol.

\section{Long-Chain n-3 Fatty Acids and the Ratio of LDL to HDL Cholesterol}

No study could be identified for the research period in which an influence of long-chain $n-3$ fatty acids on the ratio of LDL to HDL cholesterol was examined.

The evidence for a missing association of an increased dietary intake of long-chain n-3 fatty acids on the total cholesterol concentration in plasma is probable.

There is possible evidence for an increase in the $L D L$ cholesterol concentration in plasma through an increased intake of long-chain n-3 fatty acids.

Evidence-Based Guideline of the German Nutrition Society
The evidence is possible that there is no association between the intake of long-chain n-3 fatty acids and the $H D L$ cholesterol concentration in plasma.

An increased intake of long-chain $n$ - 3 fatty acids leads with convincing evidence to a reduction of the triglyceride concentration in plasma. The amounts required for that can be achieved only through the intake of supplements.

The evidence for an association between the dietary intake of long-chain n-3 fatty acids and the ratio of total to HDL cholesterol and of LDL to HDL cholesterol (lack of studies) is insufficient.

\subsubsection{Ratio of n-6 to n-3 Fatty Acids}

It has not been possible up to now to identify any studies that have taken this aspect into consideration in an appropriate manner for this guideline.

Due to a lack of studies, there is insufficient evidence for the effect of the ratio of n-6 to n-3 fatty acids on the lipoproteins and lipids in plasma.

\subsection{Trans Fatty Acids}

Compared to unsaturated fatty acids, trans fatty acids caused an increase in the concentrations of LDL cholesterol and triglycerides with a simultaneous drop in the HDL cholesterol concentration ([114] LOE Ib, [80] LOE Ia). This means that trans fatty acids have a less favourable effect on the lipoprotein profile. In the meta-analysis of 13 studies with a total of 518 persons conducted by Mozaffarian and Clarke [115] (LOE Ia), it was established that substitution of $1 \%$ of the energy in the form of trans fatty acids with MUFA or PUFA lowered triglyceride, total and LDL cholesterol concentrations. A corresponding substitution of trans fatty acids with SFA, MUFA or PUFA resulted in a significant increase in the HDL cholesterol concentration and a significant decrease in the ratio of total to HDL cholesterol. In the study by Wanders et al. [116] (LOE Ib) too, the daily intake of $21.8 \mathrm{~g}$ of industrial trans fatty acids brought about a significant increase in total and LDL cholesterol concentration and in triglyceride concentration compared to oleic acid, along with a slight reduction in the concentration of HDL cholesterol and an increase in the ratio of total to HDL cholesterol. Sundram et al. [117] (LOE $\mathrm{Ib})$ established in a randomised crossover intervention study on 11 women and 22 men with an average age of $30 \pm 8$ years that a controlled diet with $3.2 \mathrm{en} \%$ trans fatty acids (plus 9.1 en\% SFA with 6.5 en\% palmitic acid, 12.4 en\% MUFA and 5.8 en\% PUFA) lowers the concentration 
of HDL cholesterol in comparison to a controlled SFA-rich diet (13.7 en\% SFA with 12.0 en\% palmitic acid,13.6 en\% MUFA and 3.6 en\% PUFA), while it increases the concentration of LDL cholesterol, thereby worsening the ratios of total to HDL cholesterol and LDL to HDL cholesterol. This study established no influence of trans fatty acids on total cholesterol and triglyceride concentration. In a doubleblind, randomised intervention study with a parallel group design conducted on 42 healthy young men, Tholstrup et al. [118] (LOE Ib) examined how the consumption of butter rich in MUFA and trans-vaccenic acid over 5 weeks affects the blood lipids. In comparison with control butter, total cholesterol concentration was significantly lowered by $6 \%$ and HDL cholesterol concentration by $9 \%$. LDL cholesterol and triglyceride concentration remained unchanged, as did the ratio of total to HDL cholesterol. In the study by Motard-Bélanger et al. [119] (LOE Ib), in which the effect of natural trans fatty acids originating in ruminants and their industrially produced equivalents was examined on 38 healthy men, an increase in both the total as well as the LDL cholesterol concentration through both types of trans fatty acid was observed with very high intake levels of $10 \mathrm{~g}$ per day. The trans fatty acids also caused a reduction in the HDL cholesterol concentration, but there were no changes in the triglyceride concentrations.

Within the scope of the Trans Fatty Acids Collaboration (TRANSFACT) Study by Chardigny et al. [120] (LOE $\mathrm{Ib})$, the effect of trans fatty acids from natural sources was compared with those of industrial origin (both approx. $4.2 \pm 0.5 \mathrm{en} \%)$ in 19 men and 21 women with an average age of $27.6 \pm 7.1$ years. Compared to trans fatty acids from industrial sources, natural trans fatty acids significantly increased concentrations of total, LDL and HDL cholesterol as well as of triglycerides. A sex-specific evaluation showed that the effects were to be observed only with women and were statistically significant. No significant differences were observed with the industrial and natural trans fatty acids with regard to the ratio of total to HDL cholesterol. The physiologically more favourable effects of industrial trans fatty acids on the lipoprotein concentrations reported in this study could possibly be caused by the different composition of the oils used. The study participants in both groups ingested a comparatively high amount of SFA, for example, but the intake of C14:0 was approximately 7 times higher in the group with the natural fatty acids, whereas the intake of C16:0 was roughly one and a half times and of C18:1 roughly twice as high as in the group with the industrial trans fatty acids. The fact that the intake of oleic acid was roughly twice as high (5.6 vs. 3.3 en\%) among the study participants in the in- dustrial trans fatty acid group could possibly be responsible for the observed effects. The study by Chardigny et al. [120] (LOE Ib) was not included in the evidence judgement for this reason.

The evidence that an increase in the proportion of trans fatty acids in the diet increases the total and $L D L$ cholesterol concentration in plasma is convincing.

The evidence for a reduction of the HDL cholesterol concentration in plasma through an increased proportion of trans fatty acids in the diet is convincing.

The evidence is convincing that an increase in the proportion of trans fatty acids in the diet raises the triglyceride concentration in plasma.

There is convincing evidence that an increased intake of trans fatty acids with the diet raises the ratio of total to HDL cholesterol.

Due to a lack of studies, there is insufficient evidence of an influence of trans fatty acids on the ratio of $L D L$ to HDL cholesterol.

There is insufficient evidence of a different effect of industrial and natural trans fatty acids from ruminants.

\subsection{Other}

\subsubsection{CLA}

No changes in blood fats were observed in the randomised, double-blind, placebo-controlled parallel intervention study by Sluijs et al. [121] (LOE Ib) on 167 men and 179 women aged $40-70$ with $4 \mathrm{~g} c 9, t 11$-CLA daily in the form of capsules over 6 months or in the doubleblind, randomised and controlled intervention study conducted by Lambert et al. [122] (LOE Ib) on 25 healthy men and 37 healthy women with a BMI $\leq 30$ aged 21-45 with $3.9 \mathrm{~g}$ of CLA-rich capsules per day (65.9\% CLA with $29.7 \% c 9, t 11$-CLA and $30.9 \% c 10, t 12$-CLA) compared to $3.9 \mathrm{~g}$ of oleic acid-rich sunflower oil capsules per day over 12 weeks. In the randomised, single-blind multiple intervention study by Wanders et al. [116] (LOE Ib), the effect of a $c 9, t 11$-CLA-rich oil $(80 \% c 9, t 11$-CLA, $20 \% t 10, c 12-$ CLA) compared to industrial trans fatty acids and oleic acid was examined over 9 weeks on 25 healthy men and 36 healthy women. On average, $26.8 \mathrm{~g}$ CLA or $21.8 \mathrm{~g}$ industrial C18:1 trans fatty acids per day were consumed. Compared to oleic acid, total and LDL cholesterol concentration were significantly increased by CLA, the HDL cholesterol concentration slightly lowered and the ratio of total to HDL cholesterol increased; the triglyceride concentration remained unchanged. Compared to the 
studies mentioned earlier, CLA intake in the study by Wanders et al. [116] (LOE Ib) was more than 5 times higher.

The evidence for effects of CLA on the total and $L D L$ cholesterol concentration in plasma is insufficient.

There is insufficient evidence for an influence of CLA on the HDL cholesterol concentration in plasma.

There is insufficient evidence for a change of the triglyceride concentration in plasma through CLA.

The evidence for an effect of CLA on the ratio of total to $L D L$ cholesterol and of LDL to HDL cholesterol (lack of studies) is insufficient.

\subsubsection{Cholesterol}

The intake of cholesterol with the diet does not have the same effect on the LDL cholesterol concentration in all people. Based on the influence of the intake of food cholesterol on the LDL cholesterol concentration, these people can be divided into 'responders' and 'nonresponders' [123-125]. Other authors distinguish between 'low absorbers' and 'high absorbers', depending on the cholesterol absorption rate [126].

Compared to long-chain SFA, cholesterol ingested with food caused only a moderate increase in the LDL cholesterol concentration in plasma [83] (LOE Ia). The reduction of $100 \mathrm{mg}$ of dietary cholesterol per day led to a reduction in the concentration of total cholesterol of around $0.056 \mathrm{mmol} / \mathrm{l}$ (ca. $2 \mathrm{mg} / \mathrm{dl}$ ) ([127] 81 studies, 5,910 participants; [128] 17 studies, 556 adults; [83]; LOE Ia). The greatest increase in plasma cholesterol is to be expected with an increased intake of dietary cholesterol if practically no cholesterol was previously ingested with food. A marginal increase can be observed according to the meta-analysis of 27 studies with 915 participants with initial total cholesterol concentrations of $>500 \mathrm{mg} / \mathrm{dl}$ published by Hopkins [129] (LOE Ia). An increased intake of cholesterol (600 mg per day compared to $200 \mathrm{mg}$ per day) intensified the increasing effect of long-chain SFA on the LDL cholesterol concentration [130] (LOE Ib). The meta-analysis conducted by Yu-Poth et al. [21] (LOE Ia) showed that the amount of cholesterol ingested with the diet has a significant effect on the total and LDL cholesterol concentration, whereas there is no significant association with the HDL cholesterol concentration. The significance of cholesterol intake for the triglyceride concentration in plasma could not be clearly determined in this study, but according to its authors, increased cholesterol intake could possibly also lead to an increased triglyceride concentration. In addition to this, higher cho- lesterol intake caused a significant increase in the ratio of total to HDL cholesterol [128] (LOE Ia).

The significance of responders and nonresponders becomes clear in the studies conducted by Greene et al. [131, 132] (LOE Ib). In a randomised crossover study on 29 postmenopausal women and 13 men aged over 60 , the effect of consuming 3 large eggs daily (equivalent to a daily intake of $640 \mathrm{mg}$ of cholesterol) was examined in comparison to a cholesterol and fat-free substitute [131] (LOE $\mathrm{Ib})$. In addition to a higher intake of cholesterol, the intake of energy, total fat, SFA, MUFA and PUFA was also slightly increased compared to the control group. The intervention led to an increase in total, LDL and HDL cholesterol concentration, whereas the ratios of LDL to HDL cholesterol and of total to HDL cholesterol were unchanged, as was the triglyceride concentration. A detailed evaluation showed that a significant increase in total, LDL and HDL cholesterol concentration was observed only in 15-42 study participants, who were classified accordingly as hyper responders $[131,132]$ (LOE Ib). In an expanded evaluation, the effects were attributed above all to changes in the distribution and frequency of individual subfractions within the lipoprotein classes [132] (LOE Ib). In an intervention study on 53 men and 72 women aged 22-70, Wolff et al. [126] (LOE Ib) were able to show that a lowfat, low-cholesterol Mediterranean diet only resulted in a decrease in the concentrations of total, HDL and LDL cholesterol in persons classified as low cholesterol absorbers. The Mediterranean diet had no influence on the lipoproteins of persons classified as high cholesterol absorbers. The diet had no influence on the triglyceride concentration irrespective of the absorber status.

The evidence is convincing that dietary cholesterol slightly raises the total and $L D L$ cholesterol concentration in plasma. It is conceivable, however, that this effect is considerably higher among the so-called responders or high absorbers.

There is probable evidence for the lack of an association between dietary cholesterol and the HDL concentration in plasma.

The evidence for the lack of an association between dietary cholesterol and the triglyceride concentration in plasma is probable.

The evidence is probable that cholesterol ingested with the diet increases the ratio of total to HDL cholesterol.

Due to a lack of studies, there is insufficient evidence for an association between dietary cholesterol and the ratio of LDL to HDL cholesterol. 


\subsection{Need for Research - Dyslipoproteinaemia}

Contrary to the numerous findings on the effects of total fat, various fatty acids and dietary cholesterol on the concentration and composition of the lipoproteins in plasma in nutritional therapy of dyslipoproteinaemias, only a few studies are available on their effects in the longterm (multi-year) primary prevention of dyslipoproteinaemias. There continues to be a great demand for research in this area.

The importance of the subclasses of individual lipoprotein fractions and the influence of the intake of various dietary fats on these subclasses has not been sufficiently examined in intervention studies up to now, so that a need for research continues to exist here, too.

SFA should be considered more precisely in future. Although the differences in the influence of the individual, even-numbered SFA on the concentration of cholesterol in plasma depending on the chain-length are known, the influences of the odd-numbered and branched-chain SFA which are usually found as minor components still have to be clarified. To take into account matrix effects too, future studies should examine the various SFA in the context of different food sources more precisely [133].

The role of dietary cholesterol with regard to its significance in primary prevention for the total and LDL cholesterol concentration, for the ratios of total to HDL cholesterol and LDL to HDL cholesterol and finally for cardiovascular events and mortality has not been sufficiently examined either. The meaning of low absorbers and high absorbers and responders and nonresponders should be further clarified in this regard.

In perspective, intervention studies should be conducted with a larger number of study participants in order to improve the reliability of the study results. Intervention studies should also be conducted over longer periods of time, such as several years. By doing so, the long-term effects on the plasma lipids caused by the changed fat intake, as well as their significance for cardiovascular events and mortality could be determined simultaneously. In addition to a regular determination of recognised risk factors, such as the plasma lipids, compliance should also be recorded, for example, by detecting or quantifying selected fatty acids from the consumption and/or supplementation in the plasma of the study participants. The significance of compliance in the evaluation of nutritional and supplementation studies is shown in the intervention study by Bjermo et al. [134], for instance. Only when the linoleic acid concentration in the plasma of the study participants confirmed compliance with the prescribed diet could a lowering of the total and LDL cholesterol concentration, as well as the triglyceride concentration and the ratio of total to HDL cholesterol through n-6 fatty acids, be observed; in the total evaluation of all test persons, only a moderate reduction of the total cholesterol concentration was recorded. In this regard, it appears necessary to document the composition of the ingested fats and record the diet followed during the intervention more reliably and in more detail in future studies so that the results of the studies can be evaluated better.

The fat ingested with the diet can have different effects or effects of different intensity, depending on age and sex. Possible age- and sex-dependent effects of the fats ingested with the diet on the plasma lipids could therefore be examined in future research projects. Also, very little is known of the significance of the interaction of individual dietary fats with one another and with the surrounding food matrix for the plasma lipids.

In intervention studies, the influence of fat intake on the triglyceride concentration is usually examined in fasting blood. Large prospective human studies indicate, however, that the postprandial triglyceride concentration is better suited for determining the CHD risk than the concentration in the fasting blood [135-137]. Therefore, there is a definite need to examine the importance of a changed fat intake on the blood fats, in particular the triglyceride concentration, postprandially. Although it is time-consuming and difficult to standardise, it seems reasonable to find out if and to what extent the postprandial progression of the triglyceride concentration in plasma manifests itself in different people depending on the type of fatty acids ingested just as it is done with cholesterol responders and nonresponders and/or low and high cholesterol absorbers.

Numerous foods enriched with phytosterols or phytostanols have come onto the market in recent years; these contain sterols in quantities that can lower the total and LDL cholesterol concentration. The influence of phytosterols on the plasma lipids should therefore be given consideration in future evidence judgements.

Due to its possible significance as a risk factor for $\mathrm{CHD}$ $[138,139]$, so-called remnant cholesterol should also be taken into account as an independent parameter in future studies in order to determine its significance for dyslipidaemias and the development of CHD. Due to the inadequacies of the Friedewald equation in calculating the LDL cholesterol concentration (for example, an estimation of the VLDL cholesterol from the triglycerides is no 
longer possible with triglyceride concentrations in plas$\mathrm{ma}$ of $>400 \mathrm{mg} / \mathrm{dl}(>4.5 \mathrm{mmol} / \mathrm{l}))$, the direct measuring of the individual parameters will also be of importance in future studies.

\section{Quantity and Quality of Dietary Fat and Fatty Acid Intake and Primary Prevention of Hypertension}

\subsection{Total Fat}

Whether or not fat reduction leads to a change in blood pressure was examined in several randomised intervention studies in which a change in weight was the primary endpoint in most cases. In a meta-analysis of intervention studies with different fat content [25] (LOE Ia), there were no significant effects on systolic (difference between fat-reduced and control diet $=-0.56 \mathrm{~mm}$ $\mathrm{Hg}$; $95 \% \mathrm{CI}-1.52$ to 0.40 ) or diastolic blood pressure (difference $=-0.35 \mathrm{~mm} \mathrm{Hg}$; 95\% CI -0.96 to 0.26 ). This meta-analysis is dominated in terms of numbers by the WHI Dietary Modification Trial [140] (LOE Ib). In this study with 48,835 postmenopausal women, women in the intervention group were motivated to reduce their fat intake. The intervention resulted in an increase in the carbohydrate proportion (58.3 vs. 48.0 en\% after year 1; 53.9 vs. 45.9 en\% after year 6), largely at the expense of the fat proportion ( 24.3 vs. $35.1 \mathrm{en} \%$ after year $1 ; 28.8 \mathrm{vs.}$ $37.0 \mathrm{en} \%$ after year 6 ). Women in the intervention group lowered their body weight in the first year of the study slightly more than the women in the control group (mean body weight 74.0 vs. $75.9 \mathrm{~kg}$ ). Similarly, a metaanalysis of 5 studies in which diets with a moderate fat content (maximum 30 en\%) were compared to diets with a low carbohydrate content (maximum $60 \mathrm{~g} /$ day) showed no difference in blood pressure after a 12-month intervention period [141] (LOE Ia). In a meta-analysis of 9 intervention studies from 2012, a significant - but only moderate - effect on systolic (difference between fat-reduced and control diet $=-1.16 \mathrm{~mm} \mathrm{Hg} ; 95 \% \mathrm{CI}$ -1.95 to -0.37$)$ and diastolic blood pressure $(-0.83 \mathrm{~mm}$ Hg; 95\% CI -1.52 to -0.13 ) was observed [24] (LOE Ia). Although studies with the purpose of weight reduction were excluded from this meta-analysis, it remains unclear whether the effect on blood pressure was caused by the change in weight. In another meta-analysis of 18 randomised intervention studies of overweight people, medium-fat and low-fat diets ( $\leq 30 \mathrm{en} \%$ fat) were compared with low-carbohydrate diets $(\leq 45$ en $\%$ carbohydrates) [142] (LOE Ia). Overall, the studies showed no

Evidence-Based Guideline of the German Nutrition Society difference in weight loss, which means that a difference in blood pressure should mostly be independent on changes in body weight. On average, no significant difference could be observed in systolic $(-1.0 \mathrm{~mm} \mathrm{Hg}$; $95 \%$ $\mathrm{CI}-3.5$ to 1.5$)$ or diastolic $(-0.7 \mathrm{~mm} \mathrm{Hg}$; $95 \% \mathrm{CI}-1.6$ to 0.2 ) blood pressure. The interpretation of the study results is partially complicated because the observed effect is not always clearly attributable to the fat content due to other simultaneous changes to the diet. Accordingly, there are often considerable differences in the choice of consumed foods and the nutrient composition of the diets. Contrary to this, in the study by Sacks et al. [143] (LOE Ib), the intervention diets were conceived in such a way that comparable foods were to be consumed, which means that in this study, the results are attributable to the composition of the energy-supplying nutrients. Under these conditions, too, comparable changes in blood pressure were observed between 4 different weight-reduction diets with different compositions of energy-supplying nutrients.

On the basis of a meta-analysis of randomised intervention studies, the evidence for a missing association between a reduced intake of total fat and the risk of hypertension is judged as possible.

\subsection{SFA}

In intervention studies with normotensive people, no significant relations between the intake of SFA compared to $\mathrm{n}-6$ fatty acids and blood pressure could be observed [144-149]. The substitution of SFA with MUFA under isocaloric conditions had no influence on blood pressure either [27] (LOE Ib).

The results of observational studies on the intake of SFA are inconsistent. In a prospective cohort study on men, the $\mathrm{P} / \mathrm{S}$ ratio (ratio of polyunsaturated fats $(\mathrm{P})$ to saturated fats (S) in the diet) was an independent predictor for a reduction of systolic but simultaneously for an increase of diastolic blood pressure over a period of 9 years [150] (LOE IIb). In another prospective cohort study on men, no associations between SFA and PUFA independent of the total fat content of the diet and changes in body weight and hypertension risk were observed [151] (LOE IIb). In the Women's Health Study too, neither intake of SFA nor the $\mathrm{P} / \mathrm{S}$ ratio were associated with risk of hypertension ( $\mathrm{RR}$ for a comparison of extreme quintiles $=1.04 ; 95 \% \mathrm{CI} 0.97-1.11)$ [152] (LOE IIb). 
Data on SFA permit the conclusion that hypertension cannot be prevented through a reduction in the proportion of SFA. The large number of randomised intervention studies on this issue provides probable evidence for a missing association.

\subsection{MUFA}

A meta-analysis of isocaloric, randomised intervention studies with MUFA-rich compared to carbohydraterich diets showed no significant difference in systolic ( $-1.3 \mathrm{~mm} \mathrm{Hg}$; $95 \% \mathrm{CI}-0.1$ to 2.6$)$ or diastolic $(-0.9 \mathrm{~mm}$ $\mathrm{Hg}$; $95 \% \mathrm{CI}-0.1$ to 1.9 ) blood pressure. There was, however, a high degree of heterogeneity between the intervention studies, especially concerning the effect on systolic blood pressure [153] (LOE Ia).

In an intervention study with diabetes patients, a MUFA-rich diet led to no different blood pressure development in the course of 1 year compared to a carbohydrate-rich diet, with both diets having comparable effects on body weight [154] (LOE Ib). Jenkins et al. [155] (LOE Ib) did not observe any differences in blood pressure between MUFA or carbohydrate-rich diets in patients with hypercholesterolaemia.

In an intervention study, 58 men and women were allocated to either a MUFA or PUFA-rich diet group after a 17-day control diet with 19 en\% SFA. After a 5-week intervention, no differences were observed between the groups with regard to systolic blood pressure, but the change in diastolic blood pressure in the women in the MUFA group $(-2.9 \mathrm{~mm} \mathrm{Hg})$ was significantly different to that of the women in the PUFA group $(+2.4 \mathrm{~mm} \mathrm{Hg})$ [147] (LOE Ib).

In a Finnish study with 87 men and women over an intervention period of 8 weeks, no difference in blood pressure was observed with a low-fat, MUFA-rich diet (26 en\% total fat, 14 en\% MUFA, 3 en\% PUFA, 7 en\% SFA) compared to a low-fat, PUFA-rich diet (26 en\% total fat, 8 en\% MUFA, 8 en\% PUFA, 8 en\% SFA). In the same study, there was also no difference compared to a low-fat, SFA-rich diet (20 en\% total fat, 8 en\% MUFA, 3 en\% PUFA, 8 en\% SFA) [149] (LOE Ib).

In an intervention study in which fat-rich diets with a high SFA or high MUFA proportion were compared, no different effects on blood pressure could be observed [27] (LOE Ib). In the KANWU Study ${ }^{3}$ on the other hand, a MUFA-rich diet (37 en\% total fat, 21 en\% MUFA) caused a reduction in diastolic blood pressure, whereas blood pressure in the group with the SFA-rich diet (37 en $\%$ total fat, 18 en\% SFA) remained unchanged. This effect was restricted to participants with a total fat intake of $<37 \mathrm{en} \%$ [156] (LOE Ib).

The intake of MUFA was not associated with risk of hypertension in normotensive persons in the Women's Health Study (RR comparison of extreme quintiles = 1.05; 95\% CI 0.99-1.12) [152] (LOE IIb).

Due to the high heterogeneity of the intervention studies, the evidence that the substitution of carbohydrates with MUFA does not affect blood pressure is judged as probable. There is insufficient evidence to date for an association with hypertension risk when SFA or PUFA are substituted with MUFA.

\subsection{PUFA}

\subsubsection{Total PUFA}

Whereas the $\mathrm{P} / \mathrm{S}$ ratio was associated with a reduction in systolic but an increase in diastolic blood pressure in a prospective study on men over a period of 9 years [150] (LOE IIb), no associations between SFA and PUFA and hypertension risk which were independent of the total fat content of the diet or changes in body weight were observed in a prospective cohort study on men [151] (LOE IIb). In the Women's Health Study, too, neither intake of PUFA (RR for the comparison of extreme quintiles $=1.03 ; 95 \% \mathrm{CI} 0.98-1.10)$ nor the $\mathrm{P} / \mathrm{S}$ ratio were associated with risk of hypertension [152] (LOE IIb).

There are currently only a few studies on the association between the intake of total PUFA and hypertension risk. The evidence is insufficient.

\subsection{2 n-6 Fatty Acids}

No significant relations between intake of $n-6$ fatty acids compared to SFA and blood pressure were observed in intervention studies with normotensive persons [144-149].

The intake of n-6 fatty acids was not associated with hypertension risk in the Women's Health Study (RR comparison of extreme quintiles $=0.99 ; 95 \%$ CI $0.94-1.05$ ) [152] (LOE IIb).

\footnotetext{
${ }^{3}$ KANWU Study: The name of the study is made up of the starting letters of the participating study centres: Kupio, Aarhus, Naples, Wollongong and Uppsala.
} 
On the basis of a large number of randomised intervention studies, there is probable evidence for a missing association between the substitution of SFA through n-6 fatty acids and risk of hypertension in normotensive persons.

\subsection{3 n-3 Fatty Acids}

A meta-analysis of randomised studies on ALA, which involved a total of 3 studies, could not observe any effect on blood pressure [157] (LOE Ia). On the contrary, supplementation with flaxseed oil (rich in ALA) caused a more pronounced reduction of systolic and diastolic blood pressure than safflower oil (rich in LA) in an intervention study [158] (LOE Ib). All participants had dyslipoproteinaemia, but most had normal blood pressure.

There is possible evidence that there is no association between a higher intake of ALA and hypertension risk.

Meta-analyses of randomised intervention studies on long-chain n-3 fatty acids showed that supplementation with long-chain n-3 fatty acids cannot significantly lower the blood pressure of normotensive people [159-162] (LOE Ia). The meta-analysis conducted by Geleijnse et al. [161] (LOE Ia) comprised 27 intervention studies on fish oil supplementation, 10 of which had a double-blind study design (527 adults). The amount of fish oil administered was relatively high in most studies (median $=3.7 \mathrm{~g} /$ day). Taking all studies into account, fish oil supplementation in normotensive people did not significantly lower systolic blood pressure ( $-1.0 \mathrm{~mm} \mathrm{Hg}$; $95 \% \mathrm{CI}-2.4$ to 0.1$)$, but did significantly lower diastolic blood pressure by $1.2 \mathrm{~mm}$ $\mathrm{Hg}$ (95\% CI -1.9 to -0.4$)$. Results from the double-blind studies did show a weakened, non-significant effect, however (systolic $-0.3 \mathrm{~mm} \mathrm{Hg}$, diastolic $-0.7 \mathrm{~mm} \mathrm{Hg}$ ). The same result was reached in the meta-analysis by Campbell et al. [162] (LOE Ia), which covers 9 intervention studies with normotensive persons. Here, the change in blood pressure through supplementation with long-chain n-3 fatty acids compared to placebo (various vegetable oils) amounted to $-0.5 \mathrm{~mm} \mathrm{Hg}$ systolic (95\% CI -1.4 to 0.45 ) and $-0.5 \mathrm{~mm} \mathrm{Hg}$ diastolic (95\% CI -1.2 to 0.2 ).

The recently published result of a very large metaanalysis of a total of 70 intervention studies shows that with supplementation averaging $3.8 \mathrm{~g}$ per day with normotensive people, EPA/DHA significantly lower systolic $(-1.25 \mathrm{~mm} \mathrm{Hg}$; 95\% CI -2.05 to -0.46$)$ and diastolic blood pressure $(-0.62 \mathrm{~mm} \mathrm{Hg}$; $95 \%$ CI -1.22 to -0.02$)$ [163] (LOE Ia).

Evidence-Based Guideline of the German Nutrition Society
In the Women's Health Study, intake of n-3 fatty acids (ALA and long-chain n-3 fatty acids) was not associated with hypertension risk (RR comparison of extreme quintiles $=1.01$; 95\% CI 0.96-1.07) [152] (LOE IIb). By contrast, participants in the CARDIA Study (Coronary Artery Risk Development in Young Adults Study) with a high intake of long-chain n-3 fatty acids had a significantly reduced hypertension risk (hazard ratio $=0.65 ; 95 \% \mathrm{CI}$ 0.53-0.79) [164] (LOE IIb).

There is probable evidence that long-chain n-3 fatty acids have a blood pressure-reducing effect. Blood pressure-reducing effects are not possible with the amounts of long-chain $n$-3 fatty acids normally ingested with conventional foods.

\subsubsection{Ratio of n-6 to n-3 Fatty Acids}

Supplementation with flaxseed oil (54\% ALA) caused a reduction of systolic and diastolic blood pressure compared to safflower oil (74\% LA) in an intervention study with persons with dyslipoproteinaemia [158] (LOE Ib). In the Women's Health Study, on the other hand, the ratio of n-6 to n-3 fatty acids was not associated with the risk of hypertension (RR comparison of extreme quintiles = 0.98; 95\% CI 0.93-1.04) [152] (LOE IIb).

There are currently only a few studies on the association between the ratio of $n-6$ to $n-3$ fatty acids and the hypertension risk. The evidence is insufficient.

\subsection{Trans Fatty Acids}

In the Women's Health Study, a higher intake of trans fatty acids was associated with an increased risk of hypertension (RR comparison of extreme quintiles $=1.08 ; 95 \%$ CI 1.01-1.15) [152] (LOE IIb).

There is currently only one study on the association between the intake of trans fatty acids and the hypertension risk. The evidence is insufficient.

\subsection{Need for Research - Hypertension}

The effect of MUFA on the blood pressure of normotensive persons has still not been sufficiently documented. It is in particular still not clear what effect the substitution of MUFA with SFA could have. Studies on the substitution of carbohydrates with MUFA also show considerable 
heterogeneity. More studies on primary prevention conducted with a larger number of study participants under a constant weight would be desirable here.

Although a blood pressure-lowering effect of longchain n-3 fatty acids exists with hypertensive people, the effects with normotensive persons are low. It still remains questionable which effects can be achieved through amounts of long-chain n-3 fatty acids realisable through alimentary intake as pharmacological doses have been used up to now in the majority of intervention studies. Studies on primary prevention would be particularly desirable here. Possible differences between EPA and DHA have not been sufficiently investigated until now. The data situation regarding ALA is also weak; more studies for comparison with LA-rich vegetable oils would be of interest here. The influence that a substitution of ALA with SFA or MUFA could have on blood pressure has not been systematically examined until now.

So far, the effects of trans fatty acids on hypertension risk have largely not been researched. In particular, prospective cohort studies in which information on the intake of trans fatty acids is recorded could enable an improvement of the evidence situation here.

\section{Quantity and Quality of Dietary Fat and Fatty Acid Intake and Primary Prevention of Metabolic Syndrome}

Within the scope of the research, a limited number of prospective cohort and intervention studies on the association between fat intake and metabolic syndrome were found. The identified intervention studies often have the disadvantage of being interventions with different components, so that effects cannot be clearly attributed to a single exposure factor. The studies are very heterogeneous and sometimes differ greatly from one another with regard to the number of participants, age of the participants and period of observation, as well as the quality of the process.

In the research, only those studies that complied with the definition of metabolic syndrome with the criteria defined by the NCEP Expert Panel [165] and/or IDF [166] were selected. The use of different limit values in the definitions considered for metabolic syndrome can also be one of the reasons for the not-always consistent results, even though the NCEP criteria were used in the majority of studies. Apart from this, there are still uncertainties which limit values are appropriate for the various ethnic groups and permit comparison. As most of the studies were conducted among Caucasians, however, this aspect has played a minor role up to now with regard to the comparability of study results.

\subsection{Total Fat}

The results of the secondary analysis of 4 intervention studies are available in which the association between total fat intake and the development of metabolic syndrome was evaluated.

In a follow-up examination of the Dietary Intervention Study in Children (DISC), the long-term effect of a change in diet over many years was examined with the goal of reducing total fat intake and increasing the intake of dietary fibre. Nine years after the end of the intervention, no difference in the prevalence of metabolic syndrome was found among the 230 female participants aged between 25 and 28 years. SFA intake was significantly lower and dietary fibre intake significantly higher in the intervention group than in the control group. Systolic blood pressure was significantly lower in the intervention group than in the control group. The serum concentrations of the large VLDL particles were also significantly lower in the intervention group, but the other lipid parameters were not [167] (LOE Ib).

In a secondary analysis of the Finnish Diabetes Prevention Study, a significant reduction in the prevalence of metabolic syndrome was found in the intervention group compared to the control group $(\mathrm{OR}=0.62$; $95 \% \mathrm{CI} 0.40-$ $0.95)$. As the lifestyle intervention comprised a total of 5 components, including a reduction of total fat intake to $<30$ en $\%$ and of SFA to $<10 \mathrm{en} \%$, as well as an increase in dietary fibre intake and physical activity, an estimation of the sole effect of the fat reduction is hardly possible. It should also be noted that $74 \%$ of the participants already fulfilled the criteria for the existence of metabolic syndrome at the time of inclusion in the study, so that the study rather is a secondary prevention study [168] (LOE $\mathrm{Ib})$.

In an intervention study conducted in Italy on 335 adults aged between 45 and 64, the results of a 1-year lifestyle intervention with general recommendations to restrict the intake of total fat and SFA and increase the intake of dietary fibres and PUFA along with physical activity were compared with those of a control group without intervention. It was shown here that the lifestyle intervention produced a highly significant reduction in the occurrence of metabolic syndrome $(\mathrm{OR}=0.28$; $95 \%$ CI 0.18 0.44 ). This too tends to be more of a secondary prevention 
study as over $70 \%$ of the participants already had metabolic syndrome when they were included in the study [169] (LOE Ib).

In an American intervention study conducted with 179 men and 149 women over a period of 1 year, the effect of 4 concepts on the change of a defined continuous score for metabolic syndrome were compared [170]. The prevalence of metabolic syndrome was approximately $30 \%$ in the 4 groups at the start of the study. A fat-reduced diet alone ( $<30$ en $\%$ total fat and $<7$ en $\%$ SFA) as well as the combination of a fat-reduced diet and additional physical exercise lowered the score most strongly and significantly compared to the control group. After making adjustments for changes in the body fat level, however, there was no longer any difference between the 4 intervention groups [171] (LOE Ib).

In addition to this, several cohort studies with publication dates of 2001 and younger which examined the influence of the total fat proportion on the risk of metabolic syndrome were found.

In the Coronary Artery Risk Development in Young Adults (CARDIA) study with 4,192 men and women (49\% Afro-Americans) aged between 18 and 30 at the beginning of the study, a significantly increased risk for the development of metabolic syndrome $(\mathrm{RR}=1.64 ; 95 \% \mathrm{CI}$ 1.19-2.25) was observed after a mean observation period of 13.6 years in the quintile with the highest total fat intake (42-59 en\%) compared to the reference quintile 3 with a lower fat intake (36-39 en\%) [172] (LOE IIb).

In a subcohort of the Framingham Nutrition Study of 300 women with a healthy metabolism aged $30-69$ years, the incidence of metabolic syndrome over a period of 12 years in dependence on 19 nutrients was examined and evaluated by means of multivariate, logistical regression analysis under consideration of age, smoking, exercise and menopause status. Women in the highest tertile of a nutrition risk score on the basis of these 19 nutrients, which was distinguished above all by a higher total fat intake and lower dietary fibre intake, had a 3 times higher risk of developing metabolic syndrome $(\mathrm{OR}=3.0 ; 95 \% \mathrm{CI}$ 1.2-7.6) than persons in the lowest tertile [173] (LOE IIb).

In an Iranian study involving 410 men and women aged between 18 and 74 years, 71 persons ( 33 men, 38 women) developed metabolic syndrome as defined by NCEP criteria after an average observation period of 3.5 years. The best predictors proved to be hypertension, waist circumference, serum triglyceride and HDL cholesterol concentration. In the univariate analysis, there was an association between total fat intake and metabolic syndrome, which was also detectable after considering po-

Evidence-Based Guideline of the German Nutrition Society tential confounding factors such as BMI and total energy intake (OR $=3.3$; 95\% CI 1.3-8.2) [174] (LOE IIb).

Over a period averaging 8 years, Ventura et al. [175] observed 152 white girls who were 5 years old at the beginning of the study. By means of a statistical method, which included 6 risk factor variables of metabolic syndrome, the girls were divided into 4 groups at the age of 13 years: 'low risk of metabolic syndrome', 'low risk of dyslipidaemia', 'low risk of hypertension' and 'high risk of metabolic syndrome'. With regard to their diet, the groups differed only in the consumption of sugar-sweetened drinks. Regarding the other energy-supplying nutrients including fats, on the other hand, no association with the development of metabolic syndrome was found [175] (LOE IIb).

Due to the small number of prospective studies, the evidence for a positive association between the total fat proportion of the diet and the occurrence of metabolic syndrome is judged as possible.

\subsection{SFA}

No prospective study was found regarding an association between intake of SFA and risk of metabolic syndrome, but there are a few studies in which the role of SFA was observed in combination with other nutritional factors. These studies $[168,169]$ have already been presented in section 7.1.

Due to a lack of suitable studies, the evidence for an association between SFA intake and the occurrence of metabolic syndrome is judged as insufficient.

\subsection{MUFA}

No study that complied with the a priori defined criteria for the literature research could be identified.

Due to lack of studies, the evidence for an association between MUFA intake and the occurrence of metabolic syndrome is judged as insufficient.

\subsection{PUFA}

Overall, only a few intervention and cohort studies were found in which an association between PUFA intake 
and metabolic syndrome was examined. A differentiation between n-6 and n-3 fatty acids was rarely made.

In the Prevencion con Dieta Mediterranea (PREDIMED) Study, a randomised, controlled intervention study, 3 nutrition concepts were compared in the course of a year in a Spanish random sample with regard to their potential for preventing and treating metabolic syndrome. At the time of inclusion, $61.4 \%$ of the 1,224 study participants fulfilled the NCEP definition criteria for metabolic syndrome. Two dietary forms were Mediterranean, supplemented with olive oil or $30 \mathrm{~g}$ of nuts per day. A low-fat mixed diet served as control. The 3 dietary forms did not differ with regard to the first-time occurrence of metabolic syndrome. With an OR of 1.7 (95\% CI 1.1-2.6), however, the remission rate of metabolic syndrome was highest among the Mediterranean diet with nuts compared to the control group. The protective effect of a Mediterranean diet with nuts with regard to the development of metabolic syndrome should trace back to various small effects, which only explain this decline when added toghether. The change in diet was not striking but consisted essentially of an increased intake of MUFA, PUFA and dietary fibres and a lower intake of SFA [176] (LOE Ib).

In addition to the PREDIMED Study, which can be viewed as a combination of a primary and a secondary prevention study, there are only a few prospective cohort studies on this topic.

In a Finnish study with 665 participants born in the years 1942, 1947, 1952, 1957 and 1962, the n-6 fatty acid proportion of the serum lipids was inversely associated with the incidence of metabolic syndrome, which was recorded over a mean observation period of 6.4 years. This relation remained after adjustment for BMI, intake of antilipemics, smoking, alcohol and physical activity [177] (LOE IIb).

Within the scope of a population-based cohort study with 3,504 Koreans aged between 40 and 69 years, the effect of fish consumption and/or intake of n-3 fatty acids on the incidence of metabolic syndrome was examined. After an average observation period of 4 years, 602 persons (345 men and 257 women) had developed a metabolic syndrome. When evaluating n-3 fatty acid intake, men in the highest decile had an incidence of metabolic syndrome that was only half as high as that of men in the lowest decile ( $\mathrm{OR}=0.53$; 95\% CI 0.28 0.99). Unlike men, there was no association between fish consumption and n-3 fatty acid intake and the occurrence of metabolic syndrome in women [178] (LOE IIb).
In a Swedish cohort study (Uppsala Longitudinal Study of Adult Men), the fatty acid composition in plasma lipids was determined and set in relation to the development of metabolic syndrome. A total of 2,009 men aged 50 and 576 men aged 70 were examined. The factor analysis identified the following 3 main factors: a 'low linoleic acid factor', an 'SFA factor' and an 'n-3-PUFA factor'. The 'low linoleic acid factor' ( $\mathrm{OR}=1.16$; $95 \% \mathrm{CI}$ $0.96-1.40)$ and 'n-3-PUFA factor' (OR $=0.78$; 95\% CI 0.64-0.94) predicted the development of metabolic syndrome over a period of 20 years, irrespective of smoking, physical activity and BMI. The authors conclude from this that more than any other factor, the fat quality is of significance for the development of metabolic syndrome. High consumption of PUFA and low intake of SFA lower the risk of developing metabolic syndrome [179] (LOE IIb).

The evidence for an inverse association between PUFA intake and the occurrence of metabolic syndrome is judged as possible.

\subsection{Trans Fatty Acids}

No studies were found regarding this aspect.

Due to a lack of studies, the evidence for an association between the intake of trans fatty acids and the occurrence of metabolic syndrome is judged as insufficient.

\subsection{Need for Research - Metabolic Syndrome}

The number of identified studies on the intake of total fat and various fatty acids is too small and the results too inconsistent to derive meaningful associations with a higher degree of evidence. Accordingly, there is an urgent need for more studies with a prospective design in order to better understand the association between fat intake and the prevention of metabolic syndrome. Additional intervention studies would be desirable. It should be noted here, however, that because lifestyle interventions are made up of several components, it is difficult to estimate the effects of the component dietary fat.

It should also be noted that there are only a few studies on the significance of diet in childhood and adolescence for the prevention of metabolic syndrome, even though this is also of interest in light of the growing problems that are arising in people of this age. The topic has 
attracted considerably more interest in recent years, however, after it was shown that this syndrome is frequently encountered in extremely obese children and adolescents [180].

\section{Quantity and Quality of Dietary Fat and Fatty Acid Intake and Primary Prevention of CHD}

Since the first edition of the DGE guideline 'Fat Intake and Prevention of Selected Nutrition-Related Diseases' in 2006, several meta-analyses of randomised and controlled intervention studies and prospective cohort studies have been published, which take into account virtually all important studies. For the judgement of the evidence, metaanalyses or pooled analyses will mainly be used in the following chapter.

In line with the main focus of the work of the DGE on primary prevention, this new edition of the fat guideline refrains from the results of studies on secondary prevention and does not consider intervention studies and meta-analyses, which only deal with secondary prevention for the evaluation of the evidence. As no difference is made between intervention studies on primary and secondary prevention in several meta-analyses, however, the common effect estimate must be given in cases of this kind. If the results of all meta-analyses of the intervention studies on secondary prevention were included in full, harder evidence would be revealed at times.

\subsection{Total Fat}

The described influence of fatty acid intake on the plasma lipids and lipoproteins led to the notion that the risk of $\mathrm{CHD}$, too, could be reduced by lowering total fat intake. As dietary fat always consists of a mixture of various fatty acids, the sometimes opposing biological effects of the different fatty acids/groups take effect simultaneously. The possible effect of a reduction in total fat intake therefore also depends on the fatty acid pattern of the dietary fat (or its change through the intervention).

The outstanding intervention study among the few studies on primary prevention of CHD through a reduction of fat intake is the Women's Health Initiative Dietary Modification Trial [140]. The study is given special attention here, even though it is also included in the following meta-analysis.

Evidence-Based Guideline of the German Nutrition Society
In the Women's Health Initiative Dietary Modification Trial, no significant influence on the number of new cases of CHD was achieved through fat reduction of $8.2 \%$ of energy intake with negligible change to the $\mathrm{P} / \mathrm{S}$ ratio (intervention 0.7 , control 0.6 ) among 19,541 postmenopausal women over a period of 6 years. After the exclusion of study participants who already had $\mathrm{CHD}$, the hazard ratio equalled 0.94 (95\% CI 0.86-1.02) in the intervention group [140] (LOE Ib).

The meta-analysis of 4 intervention studies (including 2 on primary prevention) conducted by Mente et al. [181] (LOE Ia) showed no significant association between the reduction of total fat in a person's diet and risk of CHD $(\mathrm{RR}=1.05 ; 95 \% \mathrm{CI} 0.99-1.11)$. The 2 individual studies on primary prevention did not show any significant associations either.

The latest meta-analysis of 24 intervention studies of adults with or without a cardiovascular precondition (mainly studies on secondary prevention) from $2012 \mathrm{ex}$ amined the influence of fat reduction or modification on the risk of cardiovascular diseases [25] (LOE Ia). Although a statistically significant reduction in the risk of cardiovascular diseases of $14 \%(\mathrm{RR}=0.86$; $95 \%$ CI 0.77 $0.96)$ was established, there was no significant influence on cardiovascular mortality $(\mathrm{RR}=0.96$; $95 \%$ CI $0.82-$ 1.13 ) and cardiovascular events $(\mathrm{RR}=0.97$; $95 \%$ CI 0.87 1.08). Subanalyses indicate that this effect on the incidence of cardiovascular diseases is attributable to fat modification and not to fat reduction. The authors of the study found no clear indication of heterogeneity in different risk groups (subdivided into low, moderate and high cardiovascular risk).

A more recent meta-analysis included 146 prospective cohort studies (125 studies on primary prevention) with a mean observation period of 11 years. The results of 5 cohort studies (primary prevention) were included for the evaluation of the association between total fat intake and CHD risk. There was no association between total fat intake and risk of CHD ( $\mathrm{RR}=0.98 ; 95 \%$ CI $0.87-1.10)$ [181] (LOE IIa).

On the basis of a comparable selection of reference literature (4 studies), another meta-analysis of cohort studies (primary prevention) from 2009 could not prove a significant association between total fat intake and CHD incidence [182] (LOE IIa).

For total fat intake (without regarding fat quality), there is probable evidence for a missing association with the occurrence of $\mathrm{CHD}$ in the area of primary prevention. 


\subsection{SFA}

A meta-analysis of 9 intervention studies with substitution of SFA with PUFA (without inclusion of studies focusing on fish oil fatty acids; mainly studies on secondary prevention) and therefore a higher $\mathrm{P} / \mathrm{S}$ ratio of the diet, there was a statistically significant reduction of $\mathrm{CHD}$ risk by $17 \%(\mathrm{RR}=0.83 ; 95 \% \mathrm{CI} 0.69-1.00)$ [182] (LOE Ia). There was no significant heterogeneity between the studies and no significant effect on CHD mortality $(R R=0.84$; $95 \%$ CI $0.62-1.12$ ). A subanalysis limited to 6 studies in which a significant reduction of the serum cholesterol concentration was achieved in the intervention group showed stronger effects on $\mathrm{CHD}$ incidence $(\mathrm{RR}=0.68$; 95\% CI 0.49-0.94); CHD mortality was also statistically significantly reduced in this subanalysis $(R R=0.52 ; 95 \%$ CI 0.30-0.87) [182] (LOE Ia).

Another meta-analysis of 8 intervention studies on the influence of the substitution of SFA with PUFA the CHD risk (fatal and non-fatal) showed a significant risk reduction. With an increase of PUFA intake by 5 en $\%$, risk of CHD decreased by $10 \%(\mathrm{RR}=0.90 ; 95 \%$ CI $0.83-0.97)$ [183] (LOE Ia). There was no significant heterogeneity between the studies, most of which dealt with secondary prevention. A significantly reduced CHD mortality was also seen in secondary analyses along with a stronger effect the longer the studies lasted.

Published in 2012, the Cochrane analysis of 24 intervention studies involving adults with or without cardiovascular preconditions (mainly studies on secondary prevention) examined the influence of fat reduction or modification on the risk of cardiovascular disease [25] (LOE Ia). As already mentioned, a statistically significant reduction of the risk of cardiovascular disease of $14 \%(\mathrm{RR}=0.86 ; 95 \%$ CI 0.77-0.96) was established. The authors of this study interpret its results in such a way that a reduction in SFA (through a reduction or modification of fat in the diet) could have a protective effect against cardiovascular events, while they assess the quality of the evidence as 'moderate' (between 'high' and 'low'). The results of subanalyses indicate that this reduction of the cardiovascular risk occurred in particular in studies with fat modification (not fat reduction), as well as in studies with a duration of at least 2 years and in studies with men [25] (LOE Ia).

According to the results of the meta-analysis of cohort studies by Mente et al. [181] (LOE IIa), intake of SFA does not influence incidence of CHD. Including the results of 10 studies on primary prevention, the calculated relative risk in the highest versus the lowest intake group was not significantly different $(\mathrm{RR}=1.06$; 95\% CI 0.96-1.16).
A further meta-analysis of 9 cohort studies (primary prevention) from 2009 could not prove any significant association between intake of SFA and CHD incidence or CHD mortality either [182] (LOE IIa).

One year later, Siri-Tarino et al. [184] (LOE IIa) published a meta-analysis of 21 cohort studies (primary prevention) in which they could not find any statistically significant association between SFA intake and incidence of $\mathrm{CHD}$; the calculated relative risk was $\mathrm{RR}=1.07$ (95\% CI 0.96-1.19).

A pooled analysis of 11 cohort studies (primary prevention) used substitution models in which energy intake from SFA was substituted for energy intake from MUFA, PUFA or carbohydrates. The results showed a statistically significant lower $\mathrm{CHD}$ incidence and $\mathrm{CHD}$ mortality when SFA is replaced by PUFA. The risk of CHD decreased by $13 \%(\mathrm{RR}=0.87 ; 95 \% \mathrm{CI} 0.77-0.97)$ and risk of CHD mortality by $26 \%(\mathrm{RR}=0.74 ; 95 \%$ CI $0.61-0.89)$ per 5 -en $\%$ substitution of SFA for PUFA. When SFA was replaced with MUFA, there was a slight, non-significant increase in the risk of coronary deaths $(\mathrm{RR}=1.01 ; 95 \%$ CI $0.73-1.41$ ), whereas when SFA was replaced with carbohydrates, the risk of coronary deaths decreased slightly but not significantly ( $\mathrm{RR}=0.96$; 95\% CI 0.82-1.13) [185] (LOE IIa).

The results of a follow-up work in a Danish cohort study indicated that when SFA is substituted for carbohydrates with a lower glycaemic index, a risk reduction for myocardial infarction was possible, whereas if it is replaced by carbohydrates with a high glycaemic index, risk of myocardial infarction increased significantly [186] (LOE IIb).

It is noticeable that significant results are to be seen only in meta-analyses of cohort studies that examined the substitution of SFA with PUFA and that consistently no associations were found in the other meta-analyses, which did not control the substitution. The results in 2 of 3 available meta-analyses of intervention studies also showed a favourable effect on CHD risk through the replacement of SFA with PUFA $[182,183]$ (LOE Ia). The effect detected by Hooper et al. [25] (LOE Ia) was also attributable to fat modification (often in studies where SFA was substituted with PUFA, too).

A new meta-analysis of intervention studies, observational studies with measurement of fat intake via food, and observational studies with biomarker data involving a total of over 600,000 participants had to be revised shortly after it was first published. In the revised form [187] (LOE IIa), no significant risk relation to CHD was found in the evaluation of 20 observational studies, which assessed alimentary SFA intake. No significant relation to 
CHD risk could be determined in 8 studies that assessed SFA as a biomarker. This large study was quickly confronted by several objections against its results $[188,189]$.

The evidence for primary prevention of CHD through a reduction in the intake of SFA in exchange for PUFA is judged as probable.

The evidence for a missing association between $\mathrm{CHD}$ risk and a reduction in the intake of SFA in exchange for MUFA or carbohydrates is judged as possible.

The evidence for a missing association between CHD risk and a reduction in the intake of SFA (without specific substitution by another energy source) is judged as possible.

\subsection{MUFA}

Randomised intervention studies on the specific substitution of SFA with MUFA could not be identified.

In a meta-analysis based on the results of 4 cohort studies (primary prevention), a significantly reduced risk of CHD (incidence and mortality) was reported with increased MUFA intake $(\mathrm{RR}=0.80 ; 95 \%$ CI $0.67-0.93$ ) [181] (LOE IIa).

In another meta-analysis of 7 cohort studies (primary prevention) from 2009, no significant associations between MUFA intake and risk of $\mathrm{CHD}(\mathrm{RR}=0.87 ; 95 \% \mathrm{CI}$ $0.74-1.03)$ or CHD mortality $(\mathrm{RR}=0.85 ; 95 \%$ CI 0.60 1.20 ) could be proven [182] (LOE IIa).

In the meta-analysis conducted by Chowdhury et al. [187] (LOE IIa), no association could be determined between MUFA intake from food and the MUFA concentration of the biomarker data and CHD risk.

According to the pooled analysis of 11 cohort studies (primary prevention) by Jakobsen et al. [185] (LOE IIa), MUFA as a substitute for SFA increase the risk of CHD $(\mathrm{RR}=1.19 ; 95 \%$ CI $1.00-1.42)$, but not of CHD mortality $(\mathrm{RR}=1.01 ; 95 \% \mathrm{CI} 0.73-1.41)$. According to the authors, this increased risk can be attributed at least in part to the incomplete adjustment for trans fatty acids (trans fatty acids are contained in total MUFA).

In the meta-analysis by Schwingshackl and Hoffmann [190] (LOE IIa), no significant effect on cardiological mortality ( $\mathrm{RR}=0.96$; 95\% CI 0.89-1.04), combined cardiovascular events $(\mathrm{RR}=0.95 ; 95 \%$ CI $0.89-1.02)$ and CHD (RR $=0.99 ; 95 \%$ CI 0.93-1.06) could be found for MUFA in cohort studies. The MUFA:SFA ratio showed a significantly reduced risk only for cardiovascular mortality $(\mathrm{RR}=0.91 ; 95 \%$ CI 0.83-0.99).

Evidence-Based Guideline of the German Nutrition Society
There is possible evidence for a missing association for primary prevention of CHD through MUFA (without trans fatty acids).

\subsection{PUFA}

\subsubsection{Total PUFA}

The meta-analysis of 5 intervention studies (including 3 studies on primary prevention) by Mente et al. [181] (LOE Ia) showed no significant association between PUFA intake and risk of CHD $(\mathrm{RR}=0.94$; 95\% CI $0.87-$ 1.02).

As already explained in section 8.2 (SFA), a meta-analysis of 9 intervention studies with substitution of SFA with PUFA (excluding studies that focused on fish oil fatty acids; the majority of them were studies on secondary prevention) and thereby an increased $\mathrm{P} / \mathrm{S}$ ratio of the diet showed no significant reduction of $\mathrm{CHD}$ risk $(\mathrm{RR}=0.83$; $95 \%$ CI $0.69-1.00, p=0.050$ ). When restricted to 6 studies in which a significant reduction of the serum cholesterol concentration was achieved in the intervention group, there was a stronger lowering effect on CHD incidence $(\mathrm{RR}=0.68 ; 95 \%$ CI $0.49-0.94)$, while CHD mortality was also statistically significantly reduced $(\mathrm{RR}=0.52 ; 95 \% \mathrm{CI}$ 0.30-0.87) [182] (LOE Ia).

A meta-analysis of intervention studies on primary and secondary prevention, which specifically compared the effects of $n-6$ fatty acids (see section 8.4.2) with those of a mixture of n-3 and n- 6 fatty acids in the diet, showed significant differences in their effect on CHD risk. An increase in n- 6 fatty acid intake alone showed no effect on risk of myocardial infarction and $\mathrm{CHD}$ mortality $(\mathrm{RR}=$ 1.13 ; 95\% CI 0.84-1.53), whereas the studies with an increased intake of a mixture of n-3 and n-6 fatty acids achieved a significant risk reduction $(\mathrm{RR}=0.78 ; 95 \% \mathrm{CI}$ 0.65-0.93) [191] (LOE Ia).

Another meta-analysis of 8 intervention studies ( 3 primary prevention, 4 secondary prevention and 1 mixed) on the influence of the substitution of SFA with PUFA on CHD risk showed significant results. Per 5 en\% from PUFA, CHD risk decreased by $10 \%(\mathrm{RR}=0.90 ; 95 \% \mathrm{CI}$ 0.83-0.97) [183] (LOE Ia). Significantly reduced CHD mortality $(\mathrm{RR}=0.80 ; 95 \% \mathrm{CI} 0.65-0.98)$ and a stronger effect the longer the study lasted were also seen in secondary analyses. Closer analysis of the data from this metaanalysis by Ramsden et al. [191] came to the conclusion that in 5 of the 8 intervention studies, part of the SFA had been substituted by a mixture of n- 6 and $n-3$ fatty acids and was responsible for the reduction of the CHD risk.

Ann Nutr Metab 2015;67:141-205 
According to the results of the meta-analysis of 5 cohort studies (primary prevention) by Mente et al. [181] (LOE IIa), intake of PUFA does not influence risk of $\mathrm{CHD}$. The calculated relative risk in the highest versus the lowest intake group was not significantly different $(\mathrm{RR}=$ 1.03 ; 95\% CI 0.78-1.28).

A further meta-analysis of 6 cohort studies (primary prevention) from 2009 was not able to establish a significant association ( $\mathrm{RR}=0.97 ; 95 \% \mathrm{CI} 0.74-1.27)$ between intake of PUFA and risk of CHD either, but CHD mortality was significantly increased in the highest (vs. lowest) intake group ( $\mathrm{RR}=1.25 ; 95 \% \mathrm{CI} 1.06-1.47)$ [182] (LOE IIa).

A pooled analysis of 11 cohort studies (primary prevention) used substitution models in which energy intake from SFA was replaced by energy intake from PUFA. As already explained in section 8.2 (SFA), the results showed a statistically significantly lower $\mathrm{CHD}$ incidence and CHD mortality when SFA was replaced by PUFA. The risk of CHD dropped by $13 \%(\mathrm{RR}=0.87$; $95 \%$ CI 0.77 0.97 ) per 5 -en\% substitution of SFA with PUFA and risk of CHD mortality by $26 \%$ (RR $=0.74 ; 95 \%$ CI $0.61-0.89)$ [185] (LOE IIa).

The evidence for primary prevention of $C H D$ through an increase in the intake of PUFA with a simultaneous reduction in SFA intake (and thereby an increase in the $P / S$ ratio of the diet) is judged as probable. The use of a mixture of n-3 and n- 6 fatty acids as a substitute for SFA has proven to be particularly effective in protecting against CHD.

The evidence for a missing association between primary prevention of CHD and an increase in the intake of PUFA without substitution for SFA is judged as possible.

\subsection{2 n-6 Fatty Acids}

In the meta-analysis of intervention studies on primary and secondary prevention by Ramsden et al. [191] (LOE Ia), which was already mentioned in section 8.4.1, an increase in $n-6$ fatty acid intake alone showed no effect on the risk of the combined endpoints of non-fatal myocardial infarction and CHD mortality $(\mathrm{RR}=1.13$; 95\% CI $0.84-1.53)$.

The meta-analysis of randomised, controlled intervention studies by Chowdhury et al. [187] (LOE Ia) showed that supplementation with n-6 fatty acids had no association with CHD risk. Also, no association between n- 6 fatty acid intake from food and the n- 6 fatty acid concentration of the biomarker data and CHD risk could be identified [187] (LOE IIa). As already mentioned in sec- tion 8.2, this study was quickly confronted with several objections against its results $[188,189]$.

A meta-analysis of 3 cohort studies (primary prevention) determined no significant association between intake of LA and the risk of the occurrence of CHD $(\mathrm{RR}=1.05 ; 95 \%$ CI $0.92-1.20)$, but CHD mortality was significantly increased in the highest (vs. lowest) intake group ( $\mathrm{RR}=1.25 ; 95 \% \mathrm{CI} 1.02-1.52)$ [182] (LOE IIa). The results of the 2 biggest of the 3 studies included in the analysis matched up well, but the differences between the highest and lowest intake groups were not statistically significant in the individual studies.

A reduced CHD risk of $9 \%(\mathrm{RR}=0.91 ; 95 \%$ CI $0.84-$ 0.98 ) was also found in a meta-analysis of 5 prospective studies with persons with $5 \%$ higher LA values in the cholesterol esters of plasma as biomarker [192] (LOE IIa).

A meta-analysis of 13 cohort studies with more than 300,000 participants showed a significantly lower risk of myocardial infarction of $15 \%(\mathrm{RR}=0.85$; $95 \%$ CI $0.78-$ $0.92)$ and a significantly lower risk of fatal myocardial infarction of $21 \%(\mathrm{RR}=0.79 ; 95 \%$ CI $0.71-0.89)$ among participants in the groups with the highest intake of LA compared to the groups with the lowest intake of LA. When 5 en\% SFA in the diet were replaced with the same amount of LA, risk of myocardial infarction decreased significantly by $9 \%(\mathrm{RR}=0.91 ; 95 \%$ CI $0.87-0.96)$ and risk of a fatal myocardial infarction also significantly by $13 \%(\mathrm{RR}=0.87$; 95\% CI 0.82-0.94) [193] (LOE IIa).

The derivation of the evidence for primary prevention is based on the results of cohort studies, in particular on the latest meta-analysis by Farvid et al. [193] with the most comprehensive data situation. The vast majority of the available intervention studies were conducted on patients (secondary prevention) and several of them showed considerable methodological points of criticism.

There is possible evidence that an increased intake of $n-6$ fatty acids reduces the risk of occurrence of CHD.

\subsection{3 n-3 Fatty Acids}

The most important $n-3$ fatty acids in human nutrition are EPA and DHA in fish and ALA in vegetable oils. The first two, whose synthesis in the human body from ALA is facilitated by a certain ratio of linoleic acid to ALA in food, are biologically more effective [194, 195].

$A L A$

An intervention study from Norway on primary prevention through the supplementation of $10 \mathrm{ml}$ of linseed 
oil, equivalent to $5.5 \mathrm{~g}$ ALA, per day over 1 year showed no reduction of CHD risk [196] (LOE Ib). The high consumption of fish in Norway, which possibly does not permit any further reduction of the CHD risk, was given as the reason for this [197] (LOE Ia). In a Finnish intervention study on primary prevention, intake of ALA was increased to $5.6 \mathrm{~g}$ per day by substituting milk fat with soya oil, thus significantly reducing the frequency of CHD in men [198] (LOE Ib), but not in women [199] (LOE Ib).

The meta-analysis of randomised, controlled intervention studies conducted by Chowdhury et al. [187] (LOE Ia) showed that supplementation with ALA has no association with CHD risk. No association between ALA intake from food and the ALA concentration of the biomarker data and CHD risk could be determined either [187] (LOE IIa).

A meta-analysis of 5 prospective cohort studies (primary prevention) showed a reduction of CHD mortality of $21 \%$ through increased intake of ALA; however, it was not significant [200] (LOE IIa).

A meta-analysis from 2009 involving 4 cohort studies (primary prevention) did not establish any significant association between intake of ALA and risk of CHD or CHD mortality [182] (LOE IIa).

In the meta-analysis by Mente et al. [181] (LOE IIa) of 4 cohort studies on the primary prevention of $\mathrm{CHD}$, ALA intake did not influence CHD risk $(\mathrm{RR}=1.04 ; 95 \% \mathrm{CI}$ $0.86-1.21)$.

In the meta-analysis by Pan et al. [201] (LOE IIa) involving 27 observational studies, a significant reduction in the risk of cardiovascular events was determined in the highest (vs. lowest) ALA category (intake and biomarker $)(\mathrm{RR}=0.86 ; 95 \% \mathrm{CI} 0.77-0.97)$. There was a significant inverse association between intake of ALA and cardiovascular risk (13 studies) $(\mathrm{RR}=0.90$; 95\% CI 0.810.99). A comparable but statistically insignificant effect estimate was determined for the association between biomarkers of ALA intake (17 studies) and cardiovascular risk $(\mathrm{RR}=0.80 ; 95 \% \mathrm{CI} 0.63-1.03)$. There was considerable heterogeneity between the studies. Subanalyses for total CHD or subdivided into fatal and non-fatal CHD showed no significant results for ALA intake or for ALA biomarkers; the only exception was the significant inverse association between ALA intake and CHD mortality.

In the meta-analysis of 5 prospective studies with cholesterol ester fatty acids in plasma as biomarkers, no association was found between CHD risk and ALA intake [192] (LOE IIa).

Evidence-Based Guideline of the German Nutrition Society
There is possible evidence that an increased intake of $A L A$ reduces $C H D$ mortality.

\section{Long-Chain n-3 Fatty Acids}

There are a number of intervention studies with fish, fish oil or supplements of long-chain n-3 fatty acids in patient groups (secondary prevention) whose effects on CHD risk have been quantified in many meta-analyses. With the exception of Chowdhury et al. [187] (LOE Ia), only meta-analyses are listed here which include at least one primary prevention study in the evaluation. To pay close attention to the aspect of primary prevention, each of these primary prevention studies is described again separately from the overall result of the meta-analysis.

In addition to this, there are several meta-analyses of cohort studies on the primary prevention of CHD through long-chain n-3 fatty acids.

In a meta-analysis by Studer et al. [202] (LOE Ia), 14 randomised, controlled intervention studies (13 on secondary prevention, 1 on primary prevention) on the influence of n-3 fatty acids on cardiovascular mortality were examined. The result showed a significant reduction in cardiovascular mortality through n-3 fatty acids $(\mathrm{RR}=$ $0.68 ; 95 \%$ CI $0.52-0.90)$. The included study on primary prevention did not deliver any significant result, as it was very small $(n=156)$ and only one event occurred (1 death in the intervention group, none in the control group) during the study period; a reference for this study is not contained in the publication.

A meta-analysis of 18 intervention studies showed no significant reduction in the frequency of occurrence of cardiovascular endpoints through the administration of long-chain n-3 fatty acids [203] (LOE Ia). The results of the 2 studies on primary prevention by Brox et al. [204] and Malaguarnera et al. [205] quoted therein are not contained in their publications [204, 205] (LOE Ib); a presentation in the work of Hooper et al. [25] shows that both studies have an extremely small size and number of cases ( 1 cardiovascular event in each study).

In the meta-analysis by Mente et al. [181] (LOE Ia; 10 intervention studies including 1 on primary prevention), risk of CHD events was significantly reduced with increased intake of long-chain n-3 fatty acids $(R R=0.77$; $95 \%$ CI $0.62-0.91)$. The study concerning primary prevention [206] (LOE Ib) showed no statistically significant effects of n-3 fatty acids on cardiovascular endpoints or total mortality.

In the evaluation of the results of intervention studies by Skeaff and Miller [182] (LOE Ia), the influence of fish or long-chain n-3 fatty acids on the risk of CHD events 
was significantly inverse $(\mathrm{RR}=0.89 ; 95 \%$ CI $0.82-0.98 ; 6$ studies on secondary prevention, 1 study on primary prevention), whereas the association with $\mathrm{CHD}$ mortality was marginally not significant $(\mathrm{RR}=0.88$; $95 \%$ CI 0.76 $1.01 ; 12$ studies, of which 2 were on primary prevention). There was no significant association with the risk of sudden cardiac death, fatal or non-fatal myocardial infarction. When the results of the DART II Study were excluded, the effect estimates for CHD mortality or fatal myocardial infarction were statistically significant. The 2 individual studies on primary prevention were the one already quoted above by Yokoyama et al. [206] (LOE Ib) and the unpublished results of the study by Brox et al. [204] (LOE Ib), neither of which showed any significant results.

In a meta-analysis from 2012 with consideration of a maximum of 21 intervention studies (maximum of 2 studies on primary prevention), after intervention with fish and/or n-3 fatty acids, risk of cardiovascular events was reduced by $10 \%(\mathrm{RR}=0.90 ; 95 \%$ CI $0.85-0.96 ; 14$ studies), of CHD events (fatal and non-fatal) by $18 \%$ $(\mathrm{RR}=0.82 ; 95 \%$ CI $0.75-0.90 ; 12$ studies $)$ and of CHD mortality by $9 \%(\mathrm{RR}=0.91 ; 95 \%$ CI $0.83-0.99 ; 13$ studies $)$ [207] (LOE Ia). The results of the 2 primary prevention studies showed no statistically significant effect of the intervention [206, 208] (LOE Ib).

The meta-analysis by Kotwal et al. [209] (LOE Ia) covering 20 intervention studies examined the influence of n-3 fatty acids on combined cardiovascular events and showed a significant risk reduction for vascular deaths $(\mathrm{RR}=0.86 ; 95 \% \mathrm{CI} 0.75-0.99 ; \mathrm{p}=0.03)$. In addition to the intervention studies with supplementation of long-chain n-3 fatty acids, 3 studies with dietary intervention were also taken into account. Three studies followed a primary prevention, but without significant success [206, 208, 210] (LOE Ib).

In the evaluation of 17 randomised, controlled intervention studies (13 on secondary prevention, 4 with persons with an increased CHD risk), no significant influence on CHD risk through supplementation with longchain n-3 fatty acids could be established [187] (LOE Ia).

A pooled analysis of the results of 15 prospective cohort studies (primary prevention) and 4 intervention studies (1 primary prevention) showed that an intake of $250 \mathrm{mg}$ EPA and DHA per day in primary prevention significantly reduced risk of a fatal CHD by $36 \%$ (95\% CI 20-50\%). Higher intake levels were not associated with any additional risk reduction [197] (LOE Ia/IIa).

More recent meta-analyses of cohort studies revealed similar results. Mente et al. [181] (LOE IIa) showed a sig- nificant inverse association between intake of long-chain n-3 fatty acids and risk of CHD $(\mathrm{RR}=0.88$; 95\% CI 0.77 0.99 ) as the result of the analysis of 12 cohort studies on primary prevention.

In the meta-analysis of cohort studies conducted by Skeaff and Miller [182] (LOE IIa) on the other hand, the inverse association between the intake of fish/fish oil or long-chain n-3 fatty acids and all CHD events, as well as non-fatal CHD and sudden cardiac death was not statistically significant, but there was a significant inverse association for total fatal CHD ( $\mathrm{RR}=0.82$; $95 \%$ CI $0.71-$ $0.94)$.

The effects of long-chain n-3 fatty acids were examined in a meta-analysis of prospective observational studies. On the basis of the 3 available studies on sudden cardiac death, it was shown that an intake of $>250 \mathrm{mg}$ per day as opposed to an intake of $<250 \mathrm{mg}$ led to a significant risk reduction of $35 \%(\mathrm{RR}=0.65 ; 95 \% \mathrm{CI} 0.54-0.79)$. The reductions of the risks of fatal and non-fatal coronary events were not significant in the 5 studies available on this subject [211] (LOE IIa).

In a meta-analysis of 8 prospective observational studies, there proved to be a significant relation between an increasing intake of EPA and DHA up to $200 \mathrm{mg}$ per day and a declining risk of cardiac, cardiovascular and sudden deaths ( $\mathrm{RR}=0.64$; 95\% CI 0.46-0.89) [212] (LOE IIa).

A meta-analysis of observational studies found a significantly lowered CHD risk with an increased intake of long-chain $\mathrm{n}-3$ fatty acids $(\mathrm{RR}=0.87 ; 95 \% \mathrm{CI} 0.78-0.97)$ [187] (LOE IIa).

Other prospective studies used plasma fatty acids as reliable biomarkers for fatty acid intake, thus establishing relations to cardiovascular events. In a prospective cohort study in Finland, for example, the significantly lowest risk of an acute coronary event was determined in the quartile with the highest values for DHA and DPA in serum (RR = 0.44; 95\% CI 0.11-0.65) [213] (LOE IIb).

In a prospective observation of participants in the Physicians' Health Study, the highest concentration of longchain n-3 fatty acids in whole blood was associated with the significantly lowest risk of sudden cardiac death $(\mathrm{RR}=$ 0.10; 95\% CI 0.02-0.48) [214] (LOE IIb).

Similarly, in the case-control study embedded in the Cardiovascular Health Study it was found that persons with higher EPA and DHA values in the plasma phospholipids had a significantly lower risk of fatal CHD ( RR = 0.32; 95\% CI 0.13-0.78) [215] (LOE IIb).

A study with analysis of fatty acids in fat tissue samples also showed a significantly lower risk of cardiovascular diseases $(\mathrm{RR}=0.77 ; 95 \%$ CI 0.63-0.94) with increasing 
concentrations of DPA and DHA (primary prevention) [216] (LOE IIb).

A cohort study involving senior citizens of both sexes aged 74 years on average, which used fatty acid analyses in the plasma phospholipids as biomarker, showed significantly lower cardiovascular mortality with increasing concentrations of EPA ( $\mathrm{RR}=0.72$; 95\% CI $0.54-$ $0.96)$ and DHA ( $R R=0.66 ; 95 \%$ CI $0.49-0.89)$. The risk of total mortality was also significantly lower [217] (LOE IIb).

In the meta-analysis of 5 prospective studies with cholesterol ester fatty acids in plasma as biomarker, no significant reduction of $\mathrm{CHD}$ risk was found in combination with the long-chain n-3 fatty acids EPA and DHA [192] (LOE IIa).

A meta-analysis of observational studies with fatty acids as biomarker found no significant relation of longchain n-3 fatty acids with the CHD risk [187] (LOE IIa).

On the basis of the results of cohort studies, the following conclusions were drawn for the primary prevention of CHD:

The evidence for primary prevention of $C H D$ through the intake of long-chain $n-3$ fatty acids is judged as probable. This applies at least to an intake of up to $250 \mathrm{mg}$ EPA and DHA per day. This assessment applies irrespective of the current negative results of intervention studies on the secondary prevention of CHD through the supplementation of long-chain n-3 fatty acid ethyl esters [187].

\subsection{Trans Fatty Acids}

Trans fatty acids (TFA) in the human diet originate from industrially produced, partially hardened vegetable fats/oils (IP-TFA) and fat from ruminants (R-TFA). Due to their effect on plasma lipids and plasma lipoproteins, as described in section 5.5, the CHD risk may increase as intake of trans fatty acids increases [115]. There are only cohort studies on this subject, as intervention studies on humans are out of question for ethical reasons. Possible differences in the biological effects of trans fatty acids from both processed plant fats and fats from ruminants and their consequences for the risk of CHD must be clarified.

In 2009, 3 meta-analyses of cohort studies on the association between trans fatty acid intake and risk of CHD were published.

In the meta-analysis by Mozaffarian et al. [218] (LOE IIa), the effect estimate determined from the results of 4 available prospective cohort studies (primary prevention) revealed a statistically significant increase in CHD events as the intake of trans fatty acids increased. For every 2 en\% more trans fatty acids (as a substitute for carbohydrates), risk increased by $23 \%(\mathrm{RR}=1.23$; 95\% CI 1.11-1.37). In a pooled analysis of 2 cohort studies (primary prevention), it was calculated that a 2 -en $\%$ increase in the intake of trans fatty acids at the expense of SFA, MUFA or PUFA led to an increase in CHD risk of $20 \%(\mathrm{RR}=1.20 ; 95 \% \mathrm{CI}$ $1.07-1.34), 27 \%(\mathrm{RR}=1.27 ; 95 \% \mathrm{CI} 1.14-1.42)$ and $32 \%$ $(\mathrm{RR}=1.32 ; 95 \% \mathrm{CI} 1.17-1.49)$, respectively.

In the meta-analysis by Mente et al. [181] (LOE IIa), a risk increase of $32 \%(\mathrm{RR}=1.32$; 95\% CI 1.16-1.48) was calculated for $\mathrm{CHD}$, when $\mathrm{CHD}$ events in 4 prospective cohort studies (primary prevention) were compared in the highest versus lowest trans fatty acid intake group.

In their meta-analysis of 5 cohort studies (primary prevention), Skeaff and Miller [182] (LOE IIa) showed separate estimates for all CHD events and CHD mortality. In the highest (vs. lowest) intake category for trans fatty acids, the results showed a relative risk of $R R=1.25$ (95\% CI 1.07-1.46) for all CHD events and RR $=1.32$ (95\% CI 1.08-1.61) for fatal CHD.

A meta-analysis of observational studies found a significantly elevated CHD risk for trans fatty acids ( $\mathrm{RR}=$ 1.16; 95\% CI 1.06-1.27) [187] (LOE IIa).

In a meta-analysis of 8 cohort studies (primary prevention), significant risk increases of $22 \%$ and $24 \%$ were determined in the highest (vs. lowest) intake group of trans fatty acids for CHD (RR $=1.22$; 95\% CI 1.08-1.38) and fatal $\mathrm{CHD}(\mathrm{RR}=1.24 ; 95 \% \mathrm{CI} 1.07-1.43)$, respectively. This evaluation also attempted to consider the effects of R-TFA and IP-TFA separately. The intake of R-TFA did not influence risk of CHD (RR $=0.92$; 95\% CI 0.76-1.11; 4 studies on total CHD, 1 study on fatal CHD). By contrast, the pooled relative risk for a high intake of IP-TFA was well above 1 , but did not reach statistical significance $(\mathrm{RR}=1.21 ; 95 \%$ CI $0.97-1.50 ; 2$ studies on total CHD, 1 study on fatal CHD) [219] (LOE IIa). Because of the small number of studies, the result is uncertain; the lack of effect of R-TFA could also be due to the significantly lower intake (compared with the level of IP-TFA intake).

Epidemiological studies in which trans fatty acids in biomaterials (adipose tissue, plasma phospholipids, erythrocytes) were measured as biomarkers of trans fatty acid intake are highly heterogeneous and generally have a retrospective case-control study design. The results are inconsistent, showing either an increased risk for cardiovascular diseases as the concentrations of trans fatty acids increase, or no association at all [218]. 
With regard to primary prevention the evidence for an increase in CHD risk caused by total trans fatty acids is judged as probable.

The evidence that trans fatty acids from processed plant fats and fats from ruminants have different effects on risk of $\mathrm{CHD}$ is judged as insufficient.

\subsection{Other}

In some cohort studies, there was a significant relation between increased cholesterol intake in the diet and an increased risk of myocardial infarction [220-223] (LOE IIb). This significant relation was confirmed again in a joint follow-up evaluation of 4 studies with a duration of between 10 and 20 years with a significantly raised CHD risk $(\mathrm{RR}=1.3 ; 95 \% \mathrm{CI} 1.1-1.5)$ when cholesterol intake was increased by $200 \mathrm{mg}$ per 1,000 kcal [224] (LOE IIa). In the Ireland-Boston Diet-Heart Study, people with coronary deaths had a significantly higher cholesterol intake [225] (LOE IIb).

In other cohort studies [226-232] (LOE IIb), this relation was not significant. A sufficient degree of correction for confounding factors took place only in a small number of the older studies.

The evidence is judged as possible that there is no association between cholesterol intake and CHD risk in healthy people.

\subsection{Need for Research - CHD}

Although there is probable or possible evidence regarding the importance of certain fatty acids in the diet for the development of $\mathrm{CHD}$, further studies are necessary.

SFA have a negative effect on the plasma lipoprotein profile and, in comparison to PUFA, increase the risk of coronary events. However, the adverse effect of SFA on the risk of coronary events is not always equally significant in cohort and intervention studies. Studies carried out on women are considerably underrepresented. In addition to the effect of even-numbered SFA on the risk of $\mathrm{CHD}$, the effect of odd-numbered and branched-chain SFA as minor components is still largely unknown. The effects of these should also be studied in the context of various food sources in order to take matrix effects [233] into account.

In the context of a Mediterranean diet, it is postulated that oleic acid has a cardioprotective effect. However, ac- cording to the results of the cohort studies, there is possible evidence for a lack of an association for a preventive effect of MUFA when these fatty acids are considered in isolation. Further studies are required in this regard.

Total PUFA should no longer be the subject of studies, as grouping fatty acids in this way is too poorly characterised and can no longer satisfy the current knowledge regarding the different effects of n-3 and n- 6 fatty acids.

In some cohort and intervention studies, n- 6 fatty acids have demonstrated a cardioprotective effect and in others this was not the case. In future studies, the effect of individual fatty acids, for example, linoleic acid as an isolated fatty acid, should be investigated.

There is probable evidence that the substitution of a portion of SFA with a mixture of n-6 and n-3 fatty acids has a cardioprotective effect. However, too little is known about the absolute amounts that are necessary and the ratio of these 2 types of PUFA.

With regard to primary prevention, the evidence that ALA has a cardioprotective effect can be classified as possible only. It is therefore necessary to carry out additional randomised and controlled intervention studies in particular, as well as a comparison of effectiveness with longchain n-3 fatty acids.

The conversion factor of ALA to long-chain n-3 fatty acids (EPA and DHA) and the influencing factors that affect this, for example, the ratio of LA to ALA or the simultaneous intake of long-chain n-3 fatty acids in the diet, are not characterised sufficiently.

The importance of the concentration of n-6 and n-3 fatty acids in plasma or tissues as a biomarker for the risk of CHD (e.g. Omega-3 Index; [234]) should also be substantiated further in clinical and epidemiological studies.

With regard to primary prevention, there is probable evidence that long-chain n-3 fatty acids (EPA and DHA) in fish and as a supplement lower cardiovascular and allcause mortality. However, it has not yet been clarified whether there are any differences between the effectiveness of fish and isolated long-chain n-3 fatty acids, for instance, as ethyl esters. In the current studies with fish, there is only insufficient information regarding the type of fish used and the preparation of the fish and therefore also inaccurate information regarding the effective preventive amount of long-chain n-3 fatty acids. It is unclear whether there are differences in the preventive effect of EPA and DHA and whether a particular ratio should be adhered to between these fatty acids.

There is probable evidence for the atherogenic effect of trans fatty acids as a fatty acid group. However, it must 
still be clarified whether there are differences in the biological effects of individual isomers of trans fatty acids, such as trans fatty acids from fats of ruminants and those from processed plant fats.

According to the latest findings, there is possible evidence that increased intake of cholesterol in the diet does not increase risk of CHD. Nevertheless, due to the indications of a possible increase in total mortality known from the consumption of eggs, further observational studies should be carried out regarding the effect of cholesterol intake on the risk of CHD.

\section{Quantity and Quality of Dietary Fat and Fatty Acid Intake and Primary Prevention of Stroke}

\subsection{Total Fat}

As part of the Women's Health Initiative Dietary Modification Trial, a multicentre, randomised, controlled intervention study on the primary prevention of stroke in postmenopausal women $(\mathrm{n}=48,835)$, a reduction in fat intake of 37.8-28.8 en\%, combined with an increased consumption of fruit and vegetables $(+1.1$ portions per day) and cereals ( +0.5 portions per day) and an increase in dietary fibre intake ( +2.4 g per day) did not lead to any differences in the incidence of stroke compared to the control group after an average follow-up of 8.1 years. The risk of ischaemic and haemorrhagic stroke was neither affected [140] (LOE Ib).

The meta-analysis carried out by Hooper et al. [25] (LOE Ia) includes 4 randomised controlled intervention studies with 49,246 participants from the United States and New Zealand. The studies investigated the effect of a reduced fat intake ( $<30$ en\%) linked to a reduced intake of SFA on the risk of stroke in primary prevention compared to a regular diet. The participants were healthy or had an elevated cardiovascular risk. Risk of stroke did not differ between the intervention and control groups.

The association between total fat intake and risk of stroke was also investigated in 14 cohort studies including 832-85,764 participants. The follow-up was between 5.5 and 20 years.

Two studies observed an inverse association between fat intake and risk of stroke. The age-adjusted stroke mortality in men of Japanese origin living in Hawaii decreased as the proportion of fat increased (25-45 en\%) [235] (LOE IIb). In the Framingham Heart Study, the age-adjusted cumulative incidence rate for stroke decreased as the proportion of fat increased (26-51 en\%) [236] (LOE IIb).
In further cohort studies that investigated men of Japanese origin in Hawaii $[237,238]$ (LOE IIb) as well as cohorts in Japan $[239,240]$ (LOE IIb), the United States [241-243], (LOE IIb), Sweden [244-246] (LOE IIb) and the United Kingdom [247] (LOE IIb), no associations were identified between total fat intake and total risk of stroke [237, 241, 244, 246, 247] (LOE IIb) as well as risk of ischaemic [237-243, 245, 246] (LOE IIb) and haemorrhagic [236-238, 241, 242, 246, 248] (LOE IIb) stroke.

There is probable evidence that total fat intake does not influence the risk of ischaemic and haemorrhagic stroke.

\subsection{SFA}

In a meta-analysis of 4 intervention studies with a total of 10,315 participants, Hooper et al. [25] (LOE Ia) examined the effect of a fat-modified diet, in which SFA (animal fats) were substituted with MUFA and PUFA (vegetable fats) and carbohydrate-containing foods, compared to a diet with a higher proportion of SFA and a lower proportion of MUFA and PUFA. The intervention had no effect on the risk of stroke.

A meta-analysis of cohort studies published in 2010 [184] (LOE IIa) included the results of 8 cohort studies from various regions (the United States, Japan, Israel, Sweden). Due to the heterogeneity between the individual studies, the meta-analysis was based on the random effect model, which assumes that differences between the studies are random. Intake of SFA was not associated with risk of stroke. Even after exclusion of 2 studies from Japan with an extremely low intake of SFA compared to other studies and after exclusion of 2 studies with haemorrhagic stroke, no association could be determined.

Most cohort studies that were not considered in the meta-analysis mentioned before showed no association between intake of SFA and total risk of stroke [246, 247] (LOE IIb) as well as ischaemic [239, 243, 246] (LOE IIb) and haemorrhagic stroke [246, 249] (LOE IIb). Only 2 studies showed an inverse relation between intake of SFA and risk of stroke: the study by Takeya et al. [249] (LOE IIb) with regard to ischaemic stroke for the cohorts living in Japan and the study by Yamagishi et al. [250] (LOE IIb) for total stroke as well as for ischaemic and haemorrhagic stroke.

The underlying studies provide probable evidence that the intake of SFA does not influence the risk of stroke. 


\subsection{MUFA}

There is a single meta-analysis [190] (LOE IIa) on the relation between the intake of MUFA and the risk of stroke, comprising 9 cohort studies [240-242, 245, 246, $248,251,252]$ (LOE IIb) with 312,228 participants. The meta-analysis showed no association between the intake of MUFA and the risk of stroke.

Two further cohort studies by Seino et al. [239] (LOE IIb) and Boden-Albala et al. [243] (LOE IIb) did not show an association between intake of MUFA and risk of ischaemic stroke either.

Atkinson et al. [247] (LOE IIb) investigated the relation between the intake of MUFA and PUFA and risk of stroke in men $(n=2,170)$ by using a semi-quantitative food frequency questionnaire. The authors did not find an association between the intake of unsaturated fatty acids and risk of stroke.

Studies identified to date provide probable evidence that intake of MUFA does not influence the risk of various types of stroke.

\subsection{PUFA}

\subsubsection{Total PUFA}

Nine cohort studies investigated whether intake of PUFA is associated with the risk of stroke [236, 239-243, $245,246,248]$ (LOE IIb). Most were carried out in the United States $(\mathrm{n}=4)$ and Japan $(\mathrm{n}=3)$, with 2 studies carried out in Sweden.

Intake of PUFA $[236,241,246]$ (LOE IIb) was not associated with risk of stroke. Even with differentiation between types of stroke, no relation could be shown between the intake of PUFA and the risk of ischaemic [236, 239-243, 245, 246] (LOE IIb) or haemorrhagic [236, 241, 242, 246, 248] (LOE IIb) stroke.

A further cohort study that investigated the effect of unsaturated fatty acid intake on the risk of stroke did not find an association [247] (LOE IIb).

The data available to date indicate with probable evidence that intake of PUFA as a whole does not influence risk of stroke.

\subsection{2 n-6 Fatty Acids}

Intake of $\mathrm{n}-6$ fatty acids was not associated with risk of stroke [246] (LOE IIb). If different subtypes of stroke were considered, associations between the intake of $n-6$ fatty acids and risk of ischaemic [239, 246] (LOE IIb) and haemorrhagic stroke $[246,248]$ (LOE IIb) were not observed either.

The available data indicate with possible evidence that the intake of n-6 fatty acids does not influence risk of stroke.

\subsection{3 n-3 Fatty Acids}

ALA

A meta-analysis of randomised controlled intervention studies including results of 3 trials with a total of 13,872 participants (healthy subjects, but also people with an elevated cardiovascular risk or CHD) could not demonstrate any effect of ALA on risk of stroke [203] (LOE Ia).

A meta-analysis of cohort studies published in 2012 showed neither an association between the intake of ALA and risk of stroke ( 3 studies) nor a relation between biomarkers of ALA intake and risk of stroke (2 studies). Due to the low number of studies considered, the reasons for the heterogeneity between studies could not be explained [201] (LOE IIa).

There is probable evidence for a lack of association between the intake of ALA and the risk of stroke.

\section{Long-Chain n-3 Fatty Acids}

In a meta-analysis of 6 randomised controlled intervention studies, which investigated the effectiveness of supplementing long-chain $n-3$ fatty acids ( $\geq 6$ months) on the risk of stroke in people without preexisting cardiovascular diseases $(n=17,383)$, risk of stroke did not vary between the verum and control group [203] (LOE Ia). Another metaanalysis [253] (LOE Ia) considered 2 randomised controlled intervention studies with a total of 31,181 participants. The supplementation ( 1.0 and $1.8 \mathrm{~g} /$ day) of longchain n-3 fatty acids - in 1 study in combination with statins which were also administered to the control group also showed no effects in follow-ups of 4.6 and 6.2 years. The meta-analysis carried out by Kotwal et al. [209] (LOE Ia; 46,750 participants; supplementation of $0.8-2 \mathrm{~g}$ longchain n-3 fatty acids/day over 1.5-6.2 years) was based on data from 7 randomised controlled intervention studies, 5 of which investigated secondary prevention and 2 of which investigated both primary and secondary prevention. The risk of cerebrovascular events did not vary between the verum and the control group, even when ischaemic and haemorrhagic stroke were considered separately.

The association between intake of long-chain n-3 fatty acids and risk of stroke was investigated in 3 meta-analy- 
ses [203, 253, 254] (LOE IIa). Hooper et al. [203] (LOE IIa) considered data from 4 cohort studies conducted in the United States and China. A high intake of long-chain n-3 fatty acids was not associated with a decrease in the risk of stroke. The meta-analysis carried out by Larsson et al. [254] (LOE IIa) was based on the results of 8 prospective cohort studies with a total of 242,076 participants (aged 20-84). In the category with the highest intake of longchain n-3 fatty acids, total risk of stroke and risk of ischaemic and haemorrhagic stroke did not differ from the category with the lowest intake. Only a differentiated consideration with regard to sex found a significantly lower risk of total stroke and its subtypes for women in the highest category of long-chain n-3 fatty acid intake compared to women in the lowest category. The meta-analysis carried out by Chowdhury et al. [253] (LOE IIa) included 14 prospective studies (11 cohort studies, 3 embedded casecontrol studies), which investigated the relation between the intake of long-chain n-3 fatty acids and risk of stroke, either directly through consumption surveys $(\mathrm{n}=10)$ or indirectly $(n=4)$ through the serum concentration. An association between the intake of long-chain n-3 fatty acids in the diet or the serum concentration of these fatty acids and risk of stroke was not shown, even with differentiation between ischaemic and haemorrhagic stroke. A subgroup analysis demonstrated a significantly lower risk of stroke in studies which only investigated women $(\mathrm{n}=$ $5)$, but not in studies which only included men $(n=8)$. Differences based on study design (prospective cohort studies vs. embedded case-control studies), study location (Europe vs. North America vs. Asia-Pacific) and followup ( $\geq 10$ vs. $<10$ years) could not be determined.

The Swedish Mammography Cohort Study (34,670 participants, average follow-up of 10.4 years), which was not considered in the meta-analysis by Chowdhury et al. [253], demonstrated a significant reduction in the risk of stroke with increasing intake of long-chain n-3 fatty acids. Differentiating between the subtypes of stroke, a risk decrease was also observed for cerebral infarction, but not for haemorrhagic stroke [246] (LOE IIb).

The available data show that, with probable evidence, no association exists between the intake of long-chain n-3 fatty acids and risk of stroke.

\subsection{Trans Fatty Acids}

The association between the intake of trans fatty acids and risk of stroke was investigated in 2 cohort studies, the
Nurses' Health Study [241] (LOE IIb) and the Health Professionals Follow-Up Study [242] (LOE IIb). Both were carried out in the United States in the 1980s and 1990s and were characterised by relatively large cohorts with 85,764 and 43,732 participants, respectively. There was a 14-year follow-up in both cases. The median intake of trans fatty acids was $2 \mathrm{~g} /$ day in the lowest quintile and 4 and $6 \mathrm{~g} /$ day in the highest quintile, respectively. No associations were observed between the intake of trans fatty acids and total risk of stroke and risk of any stroke subtypes.

There is insufficient evidence for an association between the intake of trans fatty acids and risk of stroke.

\subsection{Other}

Associations between cholesterol intake and risk of stroke were investigated in 8 cohort studies carried out in the United States $(\mathrm{n}=4)$, Japan $(\mathrm{n}=3)$ and Sweden $(\mathrm{n}=$ 1). The number of participants was between 2,283 and 85,764 with a follow-up of 5.5-17 years. Food intake was recorded using various methods $(5 \times$ food frequency questionnaires, $2 \times 24$-hour-recall, $1 \times$ dietary record).

In the cohort study by Larsson et al. [246] (LOE IIb) (Sweden), the incidence of stroke increased with cholesterol intake, while the other cohort studies observed no association between cholesterol intake and total risk of stroke [235, 241] (LOE IIb). The risk of ischaemic stroke only increased with cholesterol intake in the study by Larsson et al. [246] (LOE IIb), but not in the studies of Seino et al. [239] (Japan), Iso et al. [241] (USA), He et al. [242] (USA) and Boden-Albala et al. [243] (UK) (all LOE IIb), while Sauvaget et al. [240] (LOE IIb) (Japan) observed a lower risk with increasing cholesterol intake. For haemorrhagic [241, 242, 246, 248] (LOE IIb) and subarachnoid [241] (LOE IIb) stroke, dose-response relationships could not be observed.

Sauvaget et al. [240] (LOE IIb) recorded food intake using a 1-day dietary record, while the other studies predominantly used food frequency questionnaires. It is therefore uncertain whether the data on cholesterol intake from Sauvaget et al. [240] (LOE IIb) are representative of regular cholesterol intake. In a pathophysiological regard, no effects or possibly unfavourable effects can be expected with high cholesterol intake, as shown in the study by Larsson et al. [246] (LOE IIb). As most cohort studies did not identify any associations between cholesterol intake and the risk of stroke, it can be concluded that the intake of cholesterol is not relevant for the risk of stroke. 
There is probable evidence that cholesterol intake does not influence the risk of stroke.

\subsection{Need for Research - Stroke}

The meta-analysis carried out by Larsson et al. [254] showed a lower risk of stroke only with an intake of 200$400 \mathrm{mg}$ of long-chain n-3 fatty acids per day, but not with an intake of higher doses supplemented in randomised controlled intervention studies. This raises the question whether beneficial effects can be achieved through supplementation of medium doses of longchain n-3 fatty acids. Research is therefore required regarding the dose-response relationship, which should be investigated with newer methods (e.g. regression using Bayes methods instead of arbitrarily chosen cut-offs in meta-analyses).

Interestingly, in 2 subanalyses within meta-analyses of cohort studies, a lower risk of stroke was observed in women $[253,254]$. This could indicate that long-chain n-3 fatty acids may at least have a preventive effect in women. These indications should be investigated in future studies.

As the consumption of fish, particularly oily fish, reduced the risk of stroke [253] unlike the intake of longchain n-3 fatty acids as a supplement, the effect appears to be based on the complex nutritional composition of fish with, for example, vitamin $\mathrm{B}_{6}$, vitamin $\mathrm{B}_{12}$, vitamin $\mathrm{D}$, taurine and selenium, their vasoprotective effects and/ or their possible interaction with long-chain n-3 fatty acids [253]. This assumption was already postulated by $\mathrm{He}$ [255]. The protective effect could also be explained by a reduced intake of foods that increase cardiovascular risk (e.g. red meat) or the healthier lifestyle or higher socioeconomic status of fish consumers. These confounders were not sufficiently considered in all investigations into the effect of long-chain n-3 fatty acids to date [253] and should be the subject of further research.

An additional problem involves the data set of nutrient databases. The database of the US Department of Agriculture (USDA) only provides information on the longchain n-3 fatty acid content of two-thirds of all foods [256], meaning that the actual intake of these fatty acids is probably underestimated [253]. It is therefore desirable to complete the database so that the effectiveness of the nutritional intake of long-chain $n-3$ fatty acids can be correctly evaluated. Fish farming poses a particular problem here, as it has not been possible to obtain information regarding the possibly different composition of farmed products compared to those caught in the wild.
All of the randomised controlled intervention studies that investigated the effect of long-chain $n-3$ fatty acids on the risk of stroke did not consider stroke as the primary endpoint. This means that the validity of these studies with regard to the risk of stroke may not be sufficient [253]. The sample size calculation in future studies should therefore consider stroke as the primary target value.

As fish is practically the only source for long-chain n-3 fatty acids, their intake with infrequent (not daily) consumption of fish is not recorded correctly when 24 -hour recalls and 1-day dietary records are used. In future studies, therefore, food intake should be assessed with methods that take into account consumption over a longer period of time (e.g. food frequency questionnaires).

Furthermore, it would be desirable to record the risk of stroke based on incidence and not on mortality, as the higher number of events allows a more precise estimation of the relative risk.

In future meta-analyses, a differentiation between primary and secondary prevention would be desirable.

\section{Quantity and Quality of Dietary Fat and Fatty Acid Intake and Primary Prevention of Cancer}

The systematic literature reviews (SLR) of the World Cancer Research Fund (WCRF), which were published together with the 2007 report Food, Nutrition, Physical Activity and the Prevention of Cancer, represent an important milestone for evaluating the current data in the field of nutrition and cancer [257]. These reviews of the various cancer sites are very comprehensive and systematically describe each finding in the literature. They also contain many meta-analyses and dose-response analyses. Regarding the individual cancer sites, there have been updates based on newly published literature [258-261]. In each SLR, there is a section on fat and individual fatty acids and fatty acid fractions. Mechanisms of action are also presented.

Not all cancer sites are considered in this guideline. The cancer sites that are chosen are those with a high incident rate and/or those that are discussed in conjunction with energy balance (overweight) or dependence on hormones. In the following section, 8 sites (cancer in the colorectum, lung, bladder, pancreas, breast, endometrium, ovary and prostate) are therefore systematically investigated for associations with fat intake.

The evaluation of evidence on the preventive effect of fat intake for cancer is given only generally in the guideline and is not specified for each individual cancer. As 
cancers vary greatly and the individual cancers may differ from the overall assessment, it is recommended to refer to the description of the available evidence for the individual cancers.

\subsection{Total Fat}

In the Women's Health Initiative Dietary Modification Trail (WHI), a large-scale intervention study with multiple targeted nutritional changes over a period of 8 years and also including a reduction in fat intake, a 5\% - but not significant - reduction in the incidence of cancer was observed in conjunction with the lowered fat intake [262] (LOE Ib).

\section{Colorectal Cancer}

In the recent report of the WCRF using publications up to 2004, the meta-analysis of the cohort studies revealed no change in risk associated with a change in fat intake [257] (LOE IIa). For rectal carcinoma alone, there was also no risk relation observed in cohort studies. A further analysis of the relation between fat intake and risk of colorectal carcinoma was not carried out in the updated version of the SLR [259]. However, there is a metaanalysis carried out by Liu et al. [263] (LOE IIa) with 13 cohort studies in which no risk relation was observed. The evaluation of the cohort studies carried out with food diaries in Great Britain also found no risk relation [264] (LOE IIb). Food diaries are a nutritional survey method, which has fewer measurement errors than the frequency questionnaire. This study is therefore better equipped to prove existing risk relations.

\section{Lung Cancer}

In the first WCRF report from 1997, a positive relation between total fat intake and risk of lung cancer was still viewed as possible [265]. However, in the later evaluation by the WCRF, an association could no longer be observed on the basis of the 6 cohort studies that were considered [257] (LOE IIa). This conforms to the meta-analytical evaluation of 8 cohort studies, which showed no association between fat intake and risk of lung cancer [266] (LOE IIa).

\section{Bladder Cancer}

For the WCRF report from 2007, 3 cohort studies were identified, 2 of which were quantitatively analysed in a meta-analysis. The meta-analysis gave no indication of a risk relation. This was also reported in the third study
[257] (LOE IIa). A more recent analysis of the EPIC Study also found no indication of an association between fat intake and risk of bladder cancer [267] (LOE IIb).

\section{Pancreatic Cancer}

In the WCRF's second report [257] (LOE IIa), there were only a few findings regarding the relationship between fat intake and risk of pancreatic carcinoma. For the updated version published in 2011, 6 studies were consulted. The meta-analysis of these studies showed a relative risk of 1.05 (95\% CI 1.00-1.12) [260] (LOE IIa). There was a large degree of heterogeneity and only one individual study was observed in the analysis with a significantly increased relative risk. A more recent cohort study gave a risk estimate of below 1 , meaning that the metaanalysis mentioned before would result in a non-significant overall estimate if this more recent cohort study were included [268] (LOE IIb).

\section{Breast Cancer}

In 2007, there were only inconsistent indications that an increased fat intake is accompanied by an increased risk of breast cancer [257] (LOE IIa). The follow-up research was inconsistent too, although the estimate of the meta-analysis in postmenopausal women was almost significant [258] (LOE IIa). It was noteworthy in the Women's Health Initiative Dietary Modification Trial evaluation that, in women with a fat intake of over $36.8 \mathrm{en} \%$, the intervention (low-fat, high in fibre and vegetables) led to a risk reduction. For the fat intervention in general, no risk reduction was observed [258] (LOE IIa). A recently carried out meta-analysis of cohort studies [269] (LOE IIa) observed a risk increase with increasing fat intake in postmenopausal women. In the multi-ethnic cohort from Hawaii, no risk relation was observed with increasing fat intake [270] (LOE IIb). Nor was an association observed between fat intake and risk of breast cancer in postmenopausal women in the Vital Cohort [271] (LOE IIb). In the British cohorts with food diaries as a survey tool, there was also no relation between fat intake and risk of breast cancer [272] (LOE IIa).

\section{Endometrial Cancer}

In the second WCRF report, 2 cohort studies were identified on this topic, meaning the data pool was still limited [257]. Fat intake was evaluated once more in the updated version of the WCRF with regard to endometrial cancer, this time on the basis of 3 cohort studies. The quantitative meta-analysis showed a relative risk of 1.00 (95\% CI 0.96-1.04) per $10 \mathrm{~g} /$ day increase in fat intake and therefore no risk relation [261] (LOE IIa) was observed. 


\section{Ovarian Cancer}

For the second WCRF report, the results of 2 cohort studies were available in which no risk association was present [257] (LOE IIa). A systematic review of 2 cohort studies by Crane et al. [273] (LOE IIa) described a risk increase in one study [274] and no association in the other [275]. The evaluation carried out by Blank et al. [274] (LOE IIb) found an increased relative risk of 1.28 (95\% CI 1.01-1.63) in a comparison of the fifth and the first quintiles of consumption. In the Women's Health Initiative Dietary Modification Trial, a lowered relative risk of ovarian cancer was observed in the intervention arm with fat reduction in the final years of observation [262] (LOE Ib).

\section{Prostate Cancer}

The meta-analysis from the second WCRF report found no association between the level of fat intake and risk of prostate cancer [257] (LOE IIa). The EPIC Study also reports no risk association between fat intake and risk of prostate carcinoma [276] (LOE IIb). The accompanying meta-analysis of the 7 studies available at the time also showed no risk relation [276] (LOE IIa). Furthermore, both the NIH-AARP Study [277] (LOE IIb) and the Melbourne Collaborative Cohort Study [278] (LOE IIb) reported no change in risk associated with a modified fat intake.

According to the available data, there are only a few indications that fat intake controlled for body weight and/or energy is a significant factor for the incidence of cancer. Indications of a comparably small effect are present for postmenopausal breast cancer, ovarian cancer and pancreatic cancer. For these cancer sites, there are meta-analyses that demonstrate an increased risk with increasing fat intake. However, the results of the metaanalyses are currently called into question in some cases by new results from individual studies.

There is therefore probable evidence that no association exists between the level of total fat intake and risk of individual cancers.

\subsection{SFA}

The problem with investigating individual fatty acid fractions is that the effect of a particular level of consumption is not independent of the total fat intake. Ideally, studies regarding individual fatty acid fractions should therefore adjust for total fat intake. With such risk mod- els, it is investigated whether the individual components have specific effects beyond the effect of total fat intake. However, in risk models that do not control for total fat intake, it is investigated whether the individual components per se exhibit risk relations with the investigated cancer endpoints.

In the following chapters, the individual study results on fat fractions are presented, regardless of whether or not they were adjusted for total fat intake. The results in the following chapters should therefore not necessarily be interpreted as a specific effect of a particular individual fatty acid fraction independent of total fat intake.

\section{Colorectal Cancer}

The WCRF concluded as early as 1997 that SFA/animal fat may influence the risk of colorectal cancer [265]. However, a description of the studies on SFA did not take place until the second WCRF report [257] (LOE IIa). According to the report, none of the 8 studies found a risk association. In a meta-analysis of 12 cohort studies, no association was observed between SFA intake and risk of colorectal cancer [263] (LOE IIa). SFA were also studied in the 7 cohorts from Great Britain in which food diaries were used as the survey method. This analysis also showed no association between SFA intake and risk of colorectal cancer [264] (LOE IIb).

\section{Lung Cancer}

The second WCRF report analysed 5 cohort studies, none of which indicated an increased relative risk [257] (LOE IIa). Additional studies on this matter were not found.

\section{Bladder Cancer}

In the second WCRF report, 1 cohort study into SFA intake was evaluated, finding that no association exists [257] (LOE IIb). A recent study with the EPIC Study population also showed no association between SFA intake and the occurrence of bladder cancer [267] (LOE IIb).

\section{Pancreatic Cancer}

The WCRF report in 2011 was able to refer to 6 cohort studies on the association with SFA/animal fat, 5 of which were evaluated in a meta-analysis [260] (LOE IIa). The total estimate of the RR was 1.11 (95\% CI 1.011.21). However, only 1 study showed an increased risk. The studies had a high degree of heterogeneity. A more recent study showed no risk relation between SFA intake and risk of pancreatic cancer after potential measurement errors were taken into account [268] (LOE IIb). 


\section{Breast Cancer}

A risk increase caused by the increased intake of SFA/ animal fat was already classed as possible by the WCRF in 1997 [265]. This assessment was differentiated further in the latest assessment made by the WCRF working group on breast cancer. For breast cancer as a whole, no risk increase was observed. However, the overall estimate from 4 cohort studies on postmenopausal breast cancer showed an RR of 1.12 (95\% CI 1.01-1.24) for every $10 \mathrm{~g} /$ day increase in SFA intake. A meta-analysis of 6 cohort studies on postmenopausal breast cancer with a comparison of high and low SFA intake, on the other hand, showed no association between SFA intake and risk of breast cancer [257] (LOE IIa). The meta-analysis carried out by Turner [269] (LOE IIa) showed no indication of a risk relation between SFA intake and risk of breast cancer in the analysis of 27 cohort studies. In the evaluation of the Vital Cohort with over 30,000 women and postmenopausal breast cancer as endpoint, there was also no risk association with increasing SFA intake [271] (LOE IIb).

\section{Endometrial Cancer}

In the 2007 WCRF report, only 1 cohort study was cited [279] (LOE IIa) which examined the association between SFA intake and endometrial cancer. However, no risk association was found [257] (LOE IIa). In the WCRF's updated version from 2012, SFA were not covered. Therefore, the cohort study named in the updated version [280] was examined for statements regarding SFA. In the study by Cui et al. [280] (LOE IIb) with data from the Nurses' Health Study, no association was observed between SFA intake and risk of endometrial cancer.

\section{Ovarian Cancer}

The new WCRF report [257] (LOE IIa) cited 2 cohort studies that showed no association between SFA intake and risk of ovarian cancer. The NIH-AARP Study also demonstrated no risk relation [274] (LOE IIb).

\section{Prostate Cancer}

After energy adjustment, the 4 cohort studies considered in the WCRF report showed no risk relation between SFA intake and risk of prostate cancer [257] (LOE IIa). The studies published thereafter provided varying results. A study published shortly after the publication of the WCRF report showed no risk relation between SFA intake and risk of prostate cancer [276] (LOE IIb). The accompanying meta-analysis of the 7 studies available at that time also demonstrated no risk relation [276] (LOE IIa). Basset et al. [278] (LOE IIb) reported no association between SFA in- take and risk of prostate cancer in the Melbourne Collaborative Cohort Study. In the NIH-AARP Study, the evaluation on this subject also found no association when all prostate carcinomas were investigated [277] (LOE IIb). In this study, however, a positive association with the level of SFA intake was found both for advanced stage carcinomas and fatal carcinomas [277] (LOE IIb).

Several studies on prostate carcinoma were carried out in which the risk association with fatty acid concentration in plasma and serum was investigated. In the EPIC Study, results regarding many individual fatty acids with varying risk associations were first presented, which were difficult to understand [281] (LOE IIb). In a subsequent analysis of patterns in the fatty acids (treelet analysis), the picture became clearer and showed that the fatty acid pattern containing many SFA was not associated with the risk of prostate carcinoma [282] (LOE IIb). In the Multiethnic Study from Hawaii, SFA concentration in the blood was not associated with the risk of prostate carcinoma [283] (LOE IIb). In the Melbourne Collaborative Cohort Study, however, SFA concentration in the blood was positively associated with the risk of prostate carcinoma [278] (LOE IIb).

There is probable evidence of no association between the level of SFA intake and the risk of individual cancers.

\subsection{MUFA}

\section{Colorectal Cancer}

The SLR of the 2007 WCRF report showed no risk relation between MUFA intake and the risk of colorectal cancer. In the evaluation of 5 risk estimates from 4 cohort studies, a meta-analytical estimator of 0.95 (95\% CI $0.73-$ 1.24) was calculated for every $25 \mathrm{~g}$ of modified MUFA intake implying that no association was found [257] (LOE IIa). A subsequent meta-analysis of 11 cohort studies showed no association between MUFA intake and risk of colorectal cancer [263] (LOE IIa).

\section{Lung Cancer}

The SLR for the 2007 WCRF report identified 4 cohort studies on this topic. A formal meta-analysis was not carried out due to the varying information from the publications. Most of the studies found no risk relation; however, 2 studies reported increased relative risks in subgroups [257] (LOE IIa). The report does not mention the evaluation of 8 cohort studies by Smith-Warner et al. [266] (LOE IIa), which showed no association between MUFA intake and risk of lung cancer. 


\section{Bladder Cancer}

In the SLR for the 2007 WCRF report, 1 cohort study on MUFA intake was identified and showed no risk relation [257] (LOE IIb). A more recent study with data from the EPIC Study also showed no association between intake of MUFA and risk of bladder cancer [267] (LOE IIb).

\section{Pancreatic Cancer}

The most recent handling of the topic at the WCRF took place in the new edition of the SLR in 2011. Four studies were considered, with the evaluations showing no indications of a risk relation [260] (LOE IIa). A more recent study also showed no indication of a risk association between MUFA intake and risk of pancreatic cancer [268] (LOE IIb).

\section{Breast Cancer}

In the WCRF meta-analysis from 2007 based on the SLR, no risk increase was observed for breast cancer in the 4 studies included in the analysis per $10 \mathrm{~g} /$ day higher intake of MUFA ( $R R=1.00 ; 95 \%$ CI $0.94-1.07)$. In the 3 studies considered in the meta-analysis with postmenopausal breast cancer as the endpoint, a relative risk of 1.10 (95\% CI 0.96-1.23) per $10 \mathrm{~g} /$ day higher intake of MUFA was observed [257] (LOE IIa). The meta-analysis carried out by Turner [269] (LOE IIa) including 16 studies showed no risk relation. A recently published evaluation of a cohort of 30,000 postmenopausal women showed an increased risk of breast cancer with increasing MUFA intake [271] (LOE IIb).

\section{Endometrial Cancer}

The SLR for the WCRF report cited 1 cohort study, which showed no risk association [279] (LOE IIa). In the updated version of the WCRF report [261] (LOE IIa), MUFA were not covered for this carcinoma. The study by Cui et al. [280] (LOE IIb) with data from the Nurses' Health Study, which has already been mentioned in a different context, showed no risk relation.

\section{Ovarian Cancer}

In the SLR for the WCRF report from 2007, 2 cohort studies were cited; they showed no risk relation [257] (LOE IIa). The investigation of the NIH-AARP Study also showed no risk relation between MUFA intake and the risk of ovarian cancer [274] (LOE IIb).

\section{Prostate Cancer}

In the WCRF report from 2007, 3 studies were identified; they were evaluated through meta-analysis and showed an increased risk of prostate carcinoma with an increased intake of MUFA [257] (LOE IIa). Results from the EPIC Study showed no risk relation between the intake of MUFA and the risk of prostate cancer [276] (LOE IIb). The accompanying meta-analysis of the 7 studies available at the time also showed no risk relation [276] (LOE IIa). There was no risk relation in the Melbourne Collaborative Cohort Study, either [278] (LOE IIb). The results of the NIH-AARP Study also showed no risk relation between MUFA intake and risk of prostate cancer [277] (LOE IIb).

The studies which examined the concentrations of fatty acids in the blood showed no risk relation for MUFA $[278,282,283]$ (LOE IIb).

There is probable evidence of no association between the intake of MUFA and the risk of individual cancers.

\subsection{PUFA}

The intake data of individual PUFA such as longchain n-3 fatty acids are linked to the consumption of certain foods such as fish and are examined in many studies as marine n-3 fatty acids. Such an association must not necessarily apply to the biomarker data in the blood or adipose tissue, as each of these fatty acids can also be formed endogenously by desaturases and elongases from other n-3 or n- 6 fatty acids. Therefore, both for PUFA in general and for $\mathrm{n}-6$ or $\mathrm{n}-3$ fatty acids, attention should be paid to differences in the risk relations regarding intake compared to the concentrations of these fatty acids in biological material. When results vary, it cannot be ruled out that other nutrients or ingredients ingested through diet play a role considering the intake data.

\subsubsection{Total PUFA}

\section{Colorectal Cancer}

In the SLR for the WCRF report from 2007, 5 cohort studies that investigated PUFA were identified. None of the studies reported significant risk relations. A formal meta-analysis was not carried out [257] (LOE IIa). A later meta-analysis with 5 cohort studies showed no risk relation with the PUFA intake level $(\mathrm{RR}=1.10$; 95\% CI 0.91-1.34) [263] (LOE IIa).

\section{Lung Cancer}

In the SLR for the WCRF report from 2007, 4 cohort studies were cited which had investigated PUFA. None of
Wolfram et al. 
the studies showed a risk relation [257]. The meta-analysis of 8 cohort studies by Smith-Warner et al. [266] (LOE IIa), which was not included in the WCRF report, showed no association between PUFA intake and risk of lung cancer. No further studies were identified regarding this issue.

\section{Bladder Cancer}

In the SLR for the WCRF report, 1 cohort study on PUFA was identified and this showed no risk relation [257] (LOE IIa). The investigation of the EPIC Study on this matter showed no significantly elevated relative risk [267] (LOE IIb).

\section{Pancreatic Cancer}

The most recent handling of the topic at the WCRF took place in the updated version of the SLR in 2011. Four studies were consulted whose evaluations did not point to a risk relation [260] (LOE IIa). A more recent study also gave no indication of a risk relation between PUFA intake and risk of pancreatic cancer [268] (LOE IIb).

\section{Breast Cancer}

In its report, the WCRF had described a possible positive association between PUFA intake and risk of breast cancer [265]. This assessment was able to be confirmed on the basis of a SLR in the continuation of the report. This literature review demonstrated that the risk is increased with a high ratio of PUFA to SFA [257] (LOE IIa). The meta-analysis carried out by Turner [269] (LOE IIa) also showed a risk increase both for breast cancer as a whole (13 cohort studies) and postmenopausal breast cancer (9 cohort studies) with increased PUFA intake.

\section{Endometrial Cancer}

For this nutritional factor, the authors of the SLR of the WCRF report identified 1 cohort study that demonstrated no risk association [279] (LOE IIa). In the study by Cui et al. [280] (LOE IIb), no risk association between PUFA intake and the risk of endometrial cancer was observed as well.

\section{Ovarian Cancer}

The SLR for the WCRF report [257] (LOE IIa) analysed 2 cohort studies, which revealed no risk relation. The more recent investigation of the NIH-AARP Study found a significant positive risk relation between PUFA intake and the risk of ovarian cancer in the age-adjusted model, but not in the fully-adjusted model [274] (LOE IIb).

Evidence-Based Guideline of the German Nutrition Society

\section{Prostate Cancer}

According to the WCRF report, in the 2 cohort studies identified on the basis of the SLR, no association was observed between PUFA intake and the risk of prostate cancer following adjustment for other risk factors [257] (LOE IIa). The investigation of the EPIC Study published thereafter showed no risk relation between the intake of PUFA and risk of prostate cancer [276] (LOE IIb). The accompanying meta-analysis of the 7 studies available at the time, including Crowe et al.'s own study, also showed no risk relation [276] (LOE IIa). Basset et al. [278] (LOE IIb) also reported no relation between the intake of PUFA and risk of prostate cancer in the Melbourne Collaborative Cohort Study. In the NIH-AARP too, the evaluation showed no risk relation [277] (LOE IIb).

Fatty acids in plasma and serum were also investigated with regard to the risk of prostate carcinoma. In the Multiethnic Study from Hawaii, PUFA content in the blood was not associated with the risk of prostate carcinoma [283] (LOE IIb). In the Melbourne Collaborative Cohort Study, there was also no association between PUFA content in the blood and risk of prostate carcinoma [278] (LOE IIb).

For most cancers there is, with probable evidence, no association between PUFA intake and the risk of disease. With possible evidence, an increasing intake of PUFA may increase the risk of breast cancer.

\subsection{2 n-6 Fatty Acids}

Colorectal Cancer

In the SLR for the WCRF report from 2007, 5 cohort studies which investigated n- 6 fatty acids, were identified. None of the studies reported any significant risk relations. A formal meta-analysis was not carried out [257] (LOE IIa). An investigation from the Cancer Prevention StudyII Nutrition Cohort published shortly thereafter showed no association between the intake of $n-6$ fatty acids and risk of colorectal cancer in men and women in the strongly adjusted models [284] (LOE IIb). A cohort study from Singapore also showed no risk relation between colorectal carcinoma and intake of n-6 fatty acids [285] (LOE IIb). An analysis of a Japanese cohort, which focused on subgroups and involved people from health centres, gave no indication that the intake level of $n-6$ fatty acids is linked to the risk of cancer in the intestinal area [286] (LOE IIb).

\section{Lung Cancer}

In the SLR for the WCRF report from 2007, 1 cohort study was cited, which showed a reduced risk $(\mathrm{RR}=0.68$; 
95\% CI 0.46-1.0) for each increase of the $n-6$ fatty acid concentration in serum by 1 standard deviation [257] (LOE IIb). No further studies regarding this association were identified.

\section{Bladder Cancer}

No epidemiological studies could be identified regarding $n-6$ fatty acids.

\section{Pancreatic Cancer}

In the updated version of the SLR for the WCRF in 2011, n- 6 fatty acids were not covered with the exception of LA. No risk association was found for LA [260] (LOE IIa).

\section{Breast Cancer}

For the intake of n- 6 fatty acids, the SLR for the WCRF showed both a cohort study with a strong positive association and a cohort study with no risk relation. For the intake of LA, the 6 studies available as of 2005 showed no risk relation [257] (LOE IIa).

\section{Endometrial Cancer}

The authors of the SLR could identify only 1 cohort study on LA, showing no risk association [279] (LOE IIa). Further epidemiological studies on n-6 fatty acids and their relation to the risk of endometrial cancer were not found.

\section{Ovarian Cancer}

The SLR for the WCRF report [257] (LOE IIb) highlighted 1 cohort study that investigated LA and AA, with no risk relation found. No other epidemiological studies on this topic were found.

\section{Prostate Cancer}

In the WCRF report, 4 cohort studies on n- 6 fatty acids were identified, demonstrating no risk relation [257] (LOE IIa). In the Multiethnic Study from Hawaii, the level of n- 6 fatty acids in blood was not associated with the risk of prostate carcinoma [283] (LOE IIb). In the EPIC Study, n-6 fatty acids in blood were not linked to risk of prostate carcinoma [282] (LOE IIb). In their meta-analysis of 3 cohort studies, Chua et al. [287] (LOE IIa) found no association between the intake of n-6 fatty acids and risk of prostate cancer. Basset et al. [278] (LOE IIb) also reported that there was no association between the intake of n-6 fatty acids or blood concentrations of these fatty acids and risk of prostate cancer in the Melbourne Collaborative Cohort Study. The NIH-AARP also showed no association between the intake of n- 6 fatty acids and risk of prostate cancer [277] (LOE IIb). In the systematic review by Sakai et al. [288] (LOE IIa) on arachidonic acid, both studies with intake data and studies measuring the blood concentration of arachidonic acid demonstrated no association with the risk of prostate cancer.

With probable evidence, an increased intake of n-6 fatty acids is not associated with the risk of individual cancers.

\subsection{3 n-3 Fatty Acids and the Ratio of n-6 to n-3}

Fatty Acids

Gerber [289] published a compilation of studies on the relation between n-3 fatty acids and cancers in the colorectum, prostate and breast. Additional meta-analyses related to the individual cancer endpoints for this fatty acid fraction can also be found in the literature [e.g. 290, 291]. Together with the SLR of the WCRF [257], this gives a good data pool for n-3 fatty acids. Studies on n-6 fatty acids and cancers are not as common.

The data pool for the ratio of n-6 to $n-3$ fatty acids is also smaller. This may be due to the fact that there are comparatively few studies on n- 6 fatty acids and also no specific hypotheses on the mechanism of action of the ratio of n-6 to n-3 fatty acids and its relation to cancers. This question is therefore handled together with $n-3$ fatty acids here.

\section{Colorectal Cancer}

In the SLR on colorectal carcinoma for the WCRF report from 2007, no association was described between ALA intake and risk of colorectal cancer [257] (LOE IIa). For ALA, Gerber [289] (LOE IIa) identified 3 studies, one of which demonstrated a significantly elevated risk with increased ALA intake [284] (LOE IIb). In her review, Gerber [289] (LOE IIa) also cited 5 cohort studies on longchain n-3 fatty acids, which showed very different results. This included 1 study with an increased relative risk [285] (LOE IIb) and another study with a reduced relative risk [292] (LOE IIb) with higher intake of long-chain n-3 fatty acids. In a detailed analysis of its cohorts, a further study from Japan on long-chain n-3 fatty acids found indications of an inverse relation between EPA intake and the risk of proximal colon cancer in men and colon cancer (total) in women [286] (LOE IIb). The formal meta-analysis regarding the intake of n-3 fatty acids carried out by Shen et al. [290] (LOE IIa) gave no indication of a risk relation. However, the authors pointed out that, in the meta-analysis, a reduced risk of colorectal cancer was ob- 
served in men in conjunction with an increased intake of n-3 fatty acids.

A study from the Cancer Prevention Study-II Nutrition Cohort on the ratio of n-6 to n-3 fatty acids demonstrated no association with the risk of colorectal cancer [284] (LOE IIb). The Japan Public Health Center Cohort Study also investigated the ratio of n-3 to n- 6 fatty acids and found no risk relation [286] (LOE IIb).

\section{Lung Cancer}

No epidemiological studies regarding n-3 fatty acids could be identified.

\section{Bladder Cancer}

No epidemiological studies regarding n-3 fatty acids could be identified.

\section{Pancreatic Cancer}

The most recent handling of this topic by the WCRF took place in the updated version of the SLR in 2011 and dealt with ALA. The 3 studies that were consulted gave no indication of a risk relation between ALA intake and risk of pancreatic cancer. There was no separate evaluation for total n-3 fatty acids [260] (LOE IIa). In the 2007 WCRF report, no cohort studies on total n-3 fatty acids could be identified [257] (LOE IIa). A meta-analysis of 9 cohort studies showed no association between the intake of longchain n-3 fatty acids and risk of pancreatic cancer [293] (LOE IIa).

\section{Breast Cancer}

In the SLR on breast cancer for the WCRF report [257] (LOE IIa), a significantly increased risk of breast cancer with increased intake of n-3 fatty acids was reported in 1 of the 2 cohort studies. In a review of cohort studies, Gerber [289] (LOE IIa) investigated the different $n-3$ fatty acids. Three studies on long-chain n-3 fatty acids and breast cancer were cited, none of which showed a risk relation. Likewise, all 3 studies on ALA also showed no risk relation. In their meta-analysis (12 cohort studies) with data on intake and tissue biomarkers, Zheng et al. [291] (LOE IIa) also found no association between ALA and risk of breast cancer. Zheng et al. [291] (LOE IIa) reported in their meta-analysis of 10 cohort studies on n-3 fatty acids that intake is not associated with the risk of breast cancer. At the same time, Zheng et al. [291] (LOE IIa) also investigated the intake of long-chain marine n-3 fatty acids and found a lower relative risk with increased intake of these $n-3$ fatty acids (19 studies).

Evidence-Based Guideline of the German Nutrition Society
Yang et al. [294] (LOE IIa) investigated the ratio of n-3 to $\mathrm{n}-6$ fatty acids in 11 cohort studies on the basis of intake and biomarker data and found a reduced risk of breast cancer as the ratio increased.

\section{Endometrial Cancer}

No cohort studies on n-3 fatty acids could be identified in the SLR for the WCRF [257] (LOE IIa). In the study by Cui et al. [280] (LOE IIb), no association was observed between the intake of n-3 fatty acids and risk of endometrial cancer.

\section{Ovarian Cancer}

In 1 cohort study, intake of DHA and EPA was investigated with no risk relation observed [257] (LOE IIb).

\section{Prostate Cancer}

In the NIH-AARP Study, Leitzmann et al. [295] (LOE IIb) observed a risk increase with an increased intake of ALA. A risk increase for prostate carcinoma in conjunction with an increased intake of ALA could not be confirmed in the PCLO Study [296] (LOE IIb). Gerber [289] (LOE IIa) cited 4 cohort studies on ALA and prostate carcinoma, with 2 studies demonstrating risk increases, 1 study showing a risk decrease and another study showing no risk relation. Chua et al. [287] (LOE IIa) also observed no risk relation with the intake of ALA in their meta-analysis of 5 cohort studies. In the SELECT-Trial with ALA as biomarker, there was also no risk relation [297] (LOE IIb). However, Brasky et al. [297] (LOE IIa) were able to calculate increased relative risks with increased concentrations of DHA in their meta-analysis of 6 cohort studies with biomarkers; for EPA, there was no association. A meta-analytical evaluation of studies on the intake of long-chain n-3 fatty acids also showed no risk increase for prostate carcinoma with increased intake of long-chain n-3 fatty acids [287] (LOE IIa). Gerber [289] (LOE IIa) reported 2 cohort studies on long-chain $\mathrm{n}-3$ fatty acids, with 1 showing no risk relation and another showing a significantly reduced relative risk with an increased intake of long-chain n-3 fatty acids. In the PCLO Study, Brasky et al. [298] (LOE IIb) found an increased risk of prostate cancer with an increased concentration of the n-3 fatty acid DHA in the blood, but found no association with an increased concentration of the n-3 fatty acids ALA and EPA in the blood. The EPIC Study, on the other hand, reported that long-chain n-3 fatty acids in the blood were associated with an increased risk of prostate carcinoma [282] (LOE IIb). 
The Multiethnic Study from Hawaii showed no association between the ratio of $n-6$ to $n-3$ fatty acids in the blood and risk of prostate cancer [283] (LOE IIb). The ratio of n-6 to n-3 fatty acids was also investigated in the Melbourne Collaborative Cohort Study. No risk association was found when considering intake data or blood values [278] (LOE IIb).

The data regarding an association between the intake of $n$ - 3 fatty acids and the risk of cancer show a very complex picture for many types of cancer. This also applies to the results with fatty acid concentrations in the blood. The results of individual studies include both positive and negative risk relations with increasing fat intake. This picture becomes even more complex when the additional study results regarding PUFA in general are considered, whose conclusions may contradict each other, as is the case for breast and prostate cancer.

For most of the cancers that were studied, the evidence is probable that no association exists between intake of n-3 fatty acids and risk of disease. There is possible evidence that the risk of individual cancers may increase with an increased intake of $n-3$ fatty acids. With probable evidence, there is no association between the ratio of $n-6$ to $n-3$ fatty acids and risk of cancer. However, there is possible evidence that an increasing ratio of n- 6 to $n-3$ fatty acids can lead to a decrease in the risk of individual cancers.

\subsection{Trans Fatty Acids}

Judging trans fatty acids in relation to the risk of cancer does not seem useful. First, there are no indications that trans fatty acids, as opposed to the cis form, are associated with a biological mechanism of carcinogenesis. Sec-

Fig. 2. Summary of evidence evaluations regarding the relation between fat intake and primary prevention of selected nutrition-related diseases. ${ }^{*}$ In the chapter on dyslipoproteinaemia, there is insufficient evidence that trans FA from processed plant fats and fats from ruminants have different effects. ${ }^{1}$ Without energy adjustment; ${ }^{2}$ with energy adjustment; ${ }^{3}$ based on short-term supplementation; ${ }^{4}$ based on a long-term diet; ${ }^{5}$ MUFA substituted for starch; ${ }^{6}$ MUFA substituted for long-chain SFA $;{ }^{7}$ with an increased proportion of n-6 PUFA at the expense of carbohydrates; ${ }^{8}$ with an increased proportion of n-6 PUFA at the expense of other fatty acids; ${ }^{9}$ based on the potentially different effects of trans FA from processed plant fats and fats from ruminants; ${ }^{10}$ breast cancer; ${ }^{11}$ evidence evaluation for total n-3 fatty acids; ${ }^{12}$ individual cancers. FA = Fatty acids; MS = metabolic syndrome. The number of ond, the proportion of trans fatty acids in the biomarker profiles of fatty acids in the blood or tissues is small, meaning that a reliable and solid estimate of the cancer risk is unlikely. This assessment does not mean that investigations into trans fatty acids have not been and are not still carried out in long-term epidemiological studies. It does mean, however, that it is difficult to judge such risk estimates when evaluating causality.

The scientific requirements for judging the strength of the evidence regarding trans fatty acids and risk of cancer are not fulfilled. Accordingly, no judgement was made.

\subsection{Need for Research - Cancer}

The available data from the observed risk relations and the interventions that have been carried out do not allow conclusions on causal biological mechanisms. The difficulties in drawing strong conclusions for the primary prevention of cancer from the data are due to the multitude of alternative explanations for the risk relations in observational studies. In intervention studies, the multiple interventions and the consideration of preexisting diseases often do not allow clear conclusions on the effect of an individual nutritional factor on the disease itself. These issues cannot be resolved directly with new methodological approaches or by large-scale intervention studies. In an intervention study with nutritional modification, there exits the danger that the effects of the intervention on the rest of the diet with its many nutritional elements and not the intervention itself may be the cause of changes in risk. Therefore, study results may be interpreted incorrectly with regard to causality. Before an intervention study is carried out, a coherent approach will be necessary, defining all aspects of the nutritional change in a way that allows clear scientific conclusions.

arrows only indicates the strength of the data and not the degree of the risk. The table at the bottom of the figure does not apply to the results of the chapter dyslipoproteinaemia. An upward-pointing arrow denotes an increase in the concentration of lipoproteins and triglycerides in plasma (with convincing $\uparrow \uparrow \uparrow /$ probable $\uparrow \uparrow /$ possible evidence $\uparrow$ ), a downward-pointing arrow refers to a decrease in the concentration of lipoproteins and triglycerides in plasma (with convincing $\downarrow \downarrow \downarrow /$ probable $\downarrow \downarrow /$ possible evidence $\downarrow$ ). It should be noted that for example, an increase in the plasma concentration of HDL cholesterol is desired as it decreases the risk of dyslipoproteinaemia and that an increase in the plasma concentration of total and LDL cholesterol, on the other hand, is undesirable, as it increases the risk of dyslipoproteinaemia.

(For figure see next page.)
Wolfram et al. 
The available prospective studies should be evaluated more systematically than has been the case to date, with a standardised methodology so that the available data can be used for a systematic scientific assessment. A deeper understanding of the associations is also reached when the systematic analysis of the cohort studies is expanded to simultaneously consider the areas of food patterns, nutrients and biomarkers. A partial clarification of causal risk relations can also be achieved through the further histological characterisation of the malignant tumour.

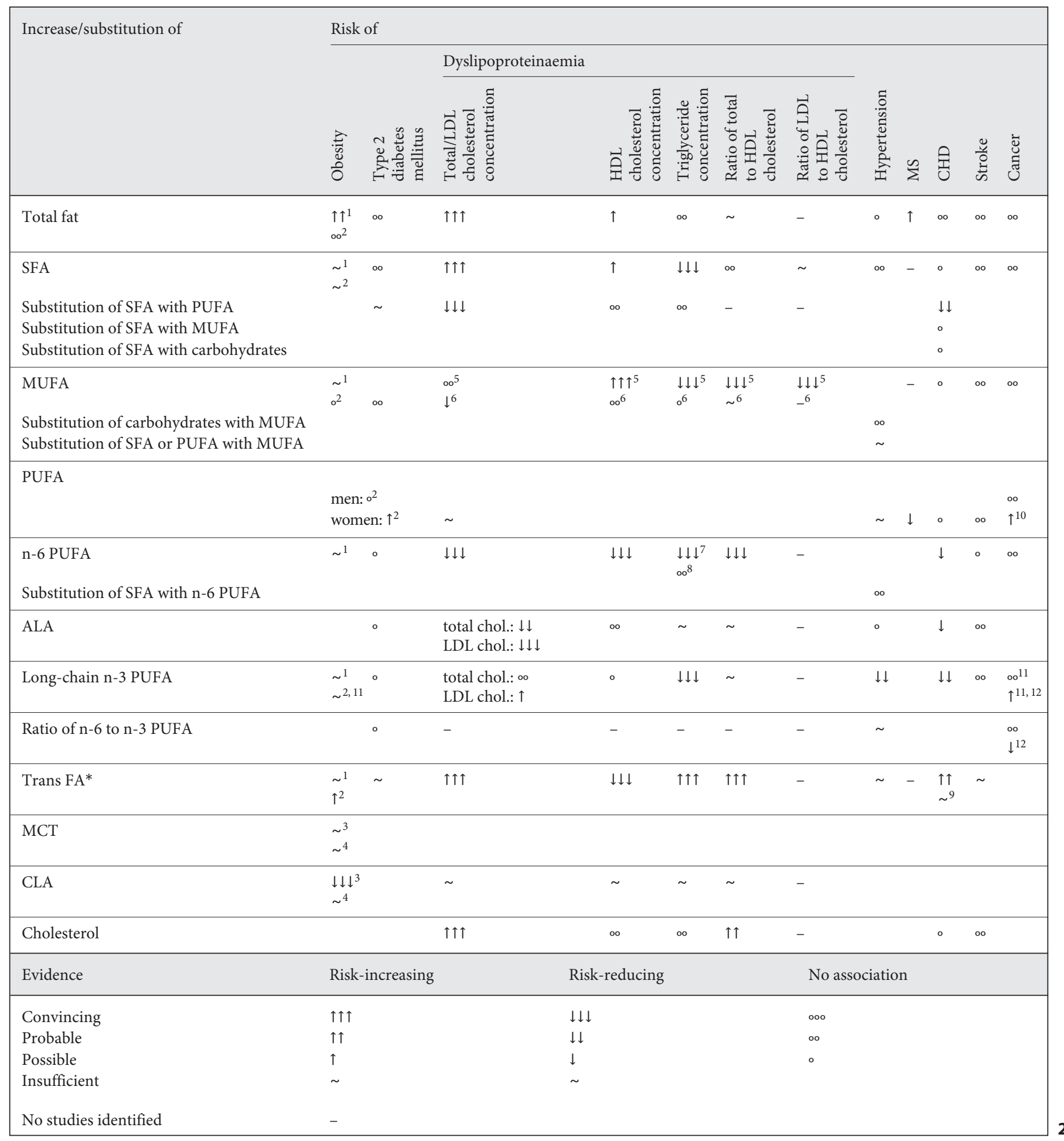


Furthermore, genetic modifications with clear functional relations should be included in the statistical models. With the Mendelian Randomisation approach, statistical methods are available, which can draw conclusions regarding the causal contribution of a substance towards the incidence of disease.

In the future, with longer life expectancy and the associated increase in cancer cases, it will become more and more important to develop approaches for secondary prevention. Diet could play a key role here. However, for the development of such lifestyle-including approaches, clear proof of the effectiveness of nutritional measures will be necessary. In Germany, there are good research opportunities for this due to the rehabilitation measures carried out by health insurers almost nationwide. One of the key questions here is whether further cancer growth can be influenced by a change in total fat intake or the selection of certain fatty acids.

\section{Summary of the Results of the Guideline on Fat Intake}

Figure 2 summarises the results of the guideline. The most significant results are as follows:

There is probable evidence that a high intake of fat with uncontrolled total energy intake favours the development of obesity. With an energy-controlled diet, however, there is probable evidence for a lack of association between fat intake and risk of obesity. There is convincing evidence that an increasing fat intake increases the plasma concentration of total and LDL cholesterol.

There is probable evidence that the substitution of SFA with PUFA lowers risk of CHD, and convincing evidence that it lowers the plasma concentration of total and LDL cholesterol.

Increasing the intake of MUFA lowers the risk of dyslipoproteinaemias, as there is convincing evidence that the plasma HDL concentration increases and the plasma triglyceride concentration and the ratios of total to HDL cholesterol and LDL to HDL cholesterol decrease.

There is probable evidence that an increased intake of long-chain n-3 fatty acids lowers risk of CHD. Furthermore, there is probable evidence that an increased intake of long-chain n-3 fatty acids lowers risk of hypertension and convincing evidence that it reduces the plasma triglyceride concentration. However, blood pressure-lowering and triglyceride-lowering effects cannot be expected with the amounts of long-chain n-3 fatty acids usually ingested by diet.

There is convincing evidence that an increased intake of trans fatty acids increases risk of dyslipoproteinaemias (with increased plasma concentrations of triglyceride as well as total and LDL cholesterol and a reduced plasma HDL cholesterol concentration). There is probable evidence that an increased intake of trans fatty acids also increases risk of CHD.

\section{Need for Research}

The data regarding the relations for the substitution of fat or fatty acids with other nutritional factors is still insufficient. Further systematic investigations would be desirable, as recommendations (for healthy people) involving a targeted substitution of nutritional components in an isocaloric diet may provide an optimum contribution to the primary prevention of nutrition-related diseases. With regard to fat intake, the question arises as to which change in risk can be expected if dietary fats are, for example, replaced by different carbohydrates or proteins, or if one fatty acid is replaced by another. The more comprehensive and systematic the knowledge in this area, the more target-oriented and effective the dietary recommendations for primary prevention of nutrition-related diseases can be.

\section{Implementation of the Guideline on Fat Intake}

Practical recommendations regarding fat intake for healthy people are derived from results where the evidence for the relation to the disease risk is classed as 'convincing' and 'probable'. The evaluation of the evidence shows that both the total amount of fat and the pattern of fatty acids must be considered in dietary recommendations.

The recommendation to limit fat intake is based on the observation that increasing total fat intake increases risk of obesity, as long as total energy intake is not considered. As obesity is an established risk factor for hypertension, dyslipoproteinaemia, CHD, type 2 diabetes mellitus, stroke and some cancers, the prevention of a strong weight increase is a central goal for primary prevention, which can be supported by limiting fat intake. Fat is the nutrient with the highest energy density, which means that limiting fat intake makes it easier to limit energy intake.

Moreover, the findings of the guideline suggest that in addition to a limitation of fat intake, a modification of the fatty acid pattern in favour of unsaturated fatty acids, par- 
ticularly long-chain n-3 fatty acids, at the expense of SFA and trans fatty acids, is desirable. According to current knowledge, these measures lower the risk of dyslipoproteinaemia and CHD. The primary prevention of $\mathrm{CHD}$ through long-chain n-3 fatty acids in the diet is considered confirmed, regardless of the failures of more recent studies regarding secondary prevention, which could be attributed to the simultaneous treatment with statins or the use of long-chain n-3 fatty acid ethyl esters. The blood pressure-lowering and triglyceride-lowering effects cannot be expected with the amounts of long-chain n-3 fatty acids usually ingested by diet.

The results of this guideline support the main statements of the D-A-CH reference values for nutrient intake [299] and the DGE recommendations on food selection [300-302]. In accordance with the D-A-CH reference values, a fat-modified and moderate fat diet with around $30 \%$ ( $\mathrm{PAL}>1.4)$ to $35 \%$ ( $\mathrm{PAL}>1.7$ ) of energy in the form of fat is recommended. For the intake of fatty acids, the following reference values should be maintained: SFA 7-10 en\%, trans fatty acids $<1$ en\%, PUFA (sum of n-6 and $n-3$ fatty acids) 7 en $\%$ to maximum 10 en $\%$. The proportion of MUFA is calculated from the difference between total fat and the fatty acids mentioned earlier.
The practical implementation of the findings from this guideline and the D-A-CH reference values is best achieved by the consumption of low-fat food variants and an increased consumption of foods of plant origin. The latter contain less fat than animal products, are free of cholesterol and have a more favourable fatty acid composition than most products of animal origin. Wholemeal products should be preferred due to their dietary fibre content, while fruit and vegetables should be preferred due to their energy density, which is lower than that of foods of animal origin because of their high water and low fat content.

A diet based on wholemeal products, 5 portions of vegetables and fruit per day and low-fat variants of milk and dairy products as well as meat and meat products lowers the consumption of animal fats. Under consideration of an equal energy balance, vegetable oils with a high proportion of ALA and oily fish rich in long-chain n-3 fatty acids can therefore be additionally integrated into the diet. This favours an increase in the intake of n-3 fatty acids. Vegetable oils with a favourable ratio of linoleic acid to ALA should be preferred. Considering the German dietary habits, rapeseed oil and walnut oil best fulfill this criterion.

\section{References}

1 Deutsche Gesellschaft für Ernährung (ed): Fettzufuhr und Prävention ausgewählter ernährungsmitbedingter Krankheiten - Evidenzbasierte Leitlinie, ed 2. Bonn, 2015. https://www.dge.de/wissenschaft/leitlinien/ (accessed March 25, 2015).

2 WHO: Diet, Nutrition and the Prevention of Chronic Diseases: Report of the Joint WHO/ FAO Expert Consultation. WHO Technical Report Series 916, 2003.

-3 Hauner $\mathrm{H}$, Bechthold A, Boeing H, Brönstrup A, Buyken A, Leschik-Bonnet E, Linseisen J, Schulze M, Strohm D, Wolfram G: Evidencebased guideline of the German Nutrition Society: carbohydrate intake and prevention of nutrition-related diseases. Ann Nutr Metab 2012;60(suppl 1):1-58.

4 Jakes RW, Day NE, Luben R, Welch A, Bingham S, Mitchell J, Hennings S, Rennie K, Wareham NJ: Adjusting for energy intake - what measure to use in nutritional epidemiological studies? Int J Epidemiol 2004;33:1382-1386.

5 Bingham SA, Luben R, Welch A, Wareham N, Khaw KT, Day N: Are imprecise methods obscuring a relation between fat and breast cancer? Lancet 2003;362:212-214.

Evidence-Based Guideline of the German Nutrition Society
6 Kipnis V, Subar AF, Midthune D, Freedman LS, Ballard-Barbash R, Troiano RP, Bingham S, Schoeller DA, Schatzkin A, Carroll RJ: Structure of dietary measurement error: results of the OPEN biomarker study. Am J Epidemiol 2003; 158:14-21; discussion 22-26.

7 Schatzkin A, Kipnis V: Could exposure assessment problems give us wrong answers to nutrition and cancer questions? J Natl Cancer Inst 2004;96:1564-1565.

-8 Sheppard L, Kristal AR, Kushi LH: Weight loss in women participating in a randomized trial of low-fat diets. Am J Clin Nutr 1991;54:821-828.

-9 Donnelly JE, Sullivan DK, Smith BK, Jacobsen DJ, Washburn RA, Johnson SL, Hill JO, Mayo MS, Spaeth KR, Gibson C: Alteration of dietary fat intake to prevent weight gain: Jayhawk Observed Eating Trial. Obesity (Silver Spring) 2008;16:107-112.

10 Heitmann BL, Lissner L, Sørensen TI, Bengtsson C: Dietary fat intake and weight gain in women genetically predisposed for obesity. Am J Clin Nutr 1995;61:1213-1217.

11 Coakley EH, Rimm EB, Colditz G, Kawachi I, Willett W: Predictors of weight change in men: results from the Health Professionals Follow-Up Study. Int J Obes Relat Metab Disord 1998;22:89-96.
12 Mosca CL, Marshall JA, Grunwald GK, Cornier MA, Baxter J: Insulin resistance as a modifier of the relationship between dietary fat intake and weight gain. Int J Obes Relat Metab Disord 2004;28:803-812.

13 Colditz GA, Willett WC, Stampfer MJ, London SJ, Segal MR, Speizer FE: Patterns of weight change and their relation to diet in a cohort of healthy women. Am J Clin Nutr 1990;51:1100-1105.

14 Field AE, Willett WC, Lissner L, Colditz GA: Dietary fat and weight gain among women in the Nurses' Health Study. Obesity (Silver Spring) 2007;15:967-976.

15 Summerbell CD, Douthwaite W, Whittaker V, Ells LJ, Hillier F, Smith S, Kelly S, Edmunds LD, Macdonald I: The association between diet and physical activity and subsequent excess weight gain and obesity assessed at 5 years of age or older: a systematic review of the epidemiological evidence. Int $\mathrm{J}$ Obes (Lond) 2009;33(suppl 3):S1-S92.

-16 Lissner L, Heitmann BL, Bengtsson C: Lowfat diets may prevent weight gain in sedentary women: prospective observations from the population study of women in Gothenburg, Sweden. Obes Res 1997;5:43-48. 
17 Parker DR, Gonzalez S, Derby CA, Gans KM, Lasater TM, Carleton RA: Dietary factors in relation to weight change among men and women from two southeastern New England communities. Int J Obes Relat Metab Disord 1997;21:103-109.

-18 Forouhi NG, Sharp SJ, Du H, van der A DL, Halkjaer J, Schulze MB, Tjønneland A, Overvad K, Jakobsen MU, Boeing H, Buijsse B, Palli D, Masala G, Feskens EJ, Sørensen TI, Wareham NJ: Dietary fat intake and subsequent weight change in adults: results from the European Prospective Investigation into Cancer and Nutrition cohorts. Am J Clin Nutr 2009:90:1632-1641.

-19 Sánchez-Villegas A, Bes-Rastrollo M, Martínez-González MA, Serra-Majem L: Adherence to a Mediterranean dietary pattern and weight gain in a follow-up study: the SUN cohort. Int J Obes (Lond) 2006;30:350-358.

20 Brixval CS, Andersen LB, Heitmann BL: Fat intake and weight development from 9 to 16 years of age: the European youth heart study a longitudinal study. Obes Facts 2009;2:166170.

-21 Yu-Poth S, Zhao G, Etherton T, Naglak M, Jonnalagadda S, Kris-Etherton PM: Effects of the national cholesterol education program's step I and step II dietary intervention programs on cardiovascular disease risk factors: a meta-analysis. Am J Clin Nutr 1999;69:632646.

-22 Astrup A, Grunwald GK, Melanson EL, Saris WH, Hill JO: The role of low-fat diets in body weight control: a meta-analysis of ad libitum dietary intervention studies. Int J Obes Relat Metab Disord 2000;24:1545-1552.

-23 Astrup A, Ryan L, Grunwald GK, Storgaard M, Saris W, Melanson E, Hill JO: The role of dietary fat in body fatness: evidence from a preliminary meta-analysis of ad libitum lowfat dietary intervention studies. Br J Nutr 2000;83(suppl 1):S25-S32.

24 Hooper L, Abdelhamid A, Moore HJ, Douthwaite W, Skeaff CM, Summerbell CD: Effect of reducing total fat intake on body weight: systematic review and meta-analysis of randomised controlled trials and cohort studies. BMJ 2012;345:e7666.

-25 Hooper L, Summerbell CD, Thompson R, Sills D, Roberts FG, Moore HJ, Davey Smith G: Reduced or modified dietary fat for preventing cardiovascular disease. Cochrane $\mathrm{Da}$ tabase Syst Rev 2012;5:CD002137.

-26 Lee-Han H, Cousins M, Beaton M, McGuire V, Kriukov V, Chipman M, Boyd N: Compliance in a randomized clinical trial of dietary fat reduction in patients with breast dysplasia. Am J Clin Nutr 1988;48:575-586.

27 Jebb SA, Lovegrove JA, Griffin BA, Frost GS, Moore CS, Chatfield MD, Bluck LJ, Williams CM, Sanders TA: Effect of changing the amount and type of fat and carbohydrate on insulin sensitivity and cardiovascular risk: the RISCK (Reading, Imperial, Surrey, Cambridge, and Kings) trial. Am J Clin Nutr 2010; 92:748-758.
Rissanen AM, Heliövaara M, Knekt P, Reunanen A, Aromaa A: Determinants of weight gain and overweight in adult Finns. Eur J Clin Nutr 1991;45:419-430.

29 Sherwood NE, Jeffery RW, French SA, Hannan PJ, Murray DM: Predictors of weight gain in the pound of prevention study. Int J Obes Relat Metab Disord 2000;24:395-403.

30 Drapeau V, Després JP, Bouchard C, Allard L, Fournier G, Leblanc C, Tremblay A: Modifications in food-group consumption are related to long-term body-weight changes. Am J Clin Nutr 2004;80:29-37.

31 Butte NF, Cai G, Cole SA, Wilson TA, Fisher JO, Zakeri IF, Ellis KJ, Comuzzie AG: Metabolic and behavioral predictors of weight gain in Hispanic children: the Viva la Familia Study. Am J Clin Nutr 2007;85:1478-1485.

32 Nimptsch K, Berg-Beckhoff G, Linseisen J: Effect of dietary fatty acid intake on prospective weight change in the Heidelberg cohort of the European prospective investigation into cancer and nutrition. Public Health Nutr 2010; 13:1636-1646.

33 Bo S, Ciccone G, Guidi S, Gambino R, Durazzo M, Gentile L, Cassader M, Cavallo-Perin P, Pagano G: Diet or exercise: what is more effective in preventing or reducing metabolic alterations? Eur J Endocrinol 2008;159:685-691.

34 Vessby B, Uusitupa M, Hermansen K, Riccardi G, Rivellese AA, Tapsell LC, Nälsén C, Berglund L, Louheranta A, Rasmussen BM, Calvert GD, Maffetone A, Pedersen E, Gustafsson IB, Storlien LH: Substituting dietary saturated for monounsaturated fat impairs insulin sensitivity in healthy men and women: the KANWU Study. Diabetologia 2001;44:312319.

35 Bes-Rastrollo M, Sánchez-Villegas A, de la Fuente C, de Irala J, Martinez JA, MartínezGonzález MA: Olive oil consumption and weight change: the SUN prospective cohort study. Lipids 2006;41:249-256.

36 Soriguer F, Almaraz MC, Ruiz-de-Adana MS, Esteva I, Linares F, García-Almeida JM, Morcillo S, García-Escobar E, Olveira-Fuster G, Rojo-Martínez G: Incidence of obesity is lower in persons who consume olive oil. Eur J Clin Nutr 2009;63:1371-1374.

37 Schwingshackl L, Strasser B, Hoffmann G: Effects of monounsaturated fatty acids on cardiovascular risk factors: a systematic review and meta-analysis. Ann Nutr Metab 2011;59: 176-186.

38 Gerhard GT, Ahmann A, Meeuws K, McMurry MP, Duell PB, Connor WE: Effects of a low-fat diet compared with those of a high-monounsaturated fat diet on body weight, plasma lipids and lipoproteins, and glycemic control in type 2 diabetes. Am J Clin Nutr 2004;80:668-673.

39 Dahm CC, Gorst-Rasmussen A, Jakobsen MU, Schmidt EB, Tjønneland A, Sørensen TI, Overvad K: Adipose tissue fatty acid patterns and changes in anthropometry: a cohort study. PLoS One 2011;6:e22587.
40 Jakobsen MU, Dethlefsen C, Due KM, Slimani N, Chajès V, May AM, Sørensen TI, Halkjær J, Tjønneland A, Clavel-Chapelon F, Boutron-Ruault MC, Fagherazzi G, Teucher B, Kaaks R, Boeing H, Schütze M, Trichopoulou A, Zylis D, Makrygiannis G, Palli D, Mattiello A, Tagliabue G, van der A DL, Buenode-Mesquita $\mathrm{HB}$, Rodríguez L, Travier $\mathrm{N}$, Molina-Montes E, Huerta JM, Barricarte A, Amiano P, Manjer J, Wirfält E, Johansson I, Hallmans G, Khaw KT, Wareham NJ, Crowe F, Romieu I, Riboli E, Peeters PH, Overvad K: Plasma phospholipid long-chain $\mathrm{n}-3$ polyunsaturated fatty acids and body weight change. Obes Facts 2011:4:312-318.

41 Whigham LD, Watras AC, Schoeller DA: Efficacy of conjugated linoleic acid for reducing fat mass: a meta-analysis in humans. Am J Clin Nutr 2007;85:1203-1211.

42 St-Onge MP, Ross R, Parsons WD, Jones PJ: Medium-chain triglycerides increase energy expenditure and decrease adiposity in overweight men. Obes Res 2003;11:395-402.

43 Papamandjaris AA, White MD, Jones PJ: Components of total energy expenditure in healthy young women are not affected after 14 days of feeding with medium-versus longchain triglycerides. Obes Res 1999;7:273-280.

44 Krotkiewski M: Value of VLCD supplementation with medium chain triglycerides. Int J Obes Relat Metab Disord 2001;25:1393-1400.

45 Tuomilehto J, Lindstrom J, Eriksson JG, Valle TT, Hämäläinen $\mathrm{H}$, Ilanne-Parikka $\mathrm{P}$, Keinänen-Kiukaanniemi S, Laakso $M$, Louheranta A, Rastas M, Salminen V, Uusitupa M: Prevention of type 2 diabetes mellitus by changes in lifestyle among subjects with impaired glucose tolerance. N Engl J Med 2001; 344:1343-1350.

-46 Knowler WC, Barrett-Connor E, Fowler SE, Hamman RF, Lachin JM, Walker EA, Nathan DM: Reduction in the incidence of type 2 diabetes with lifestyle intervention or metformin. N Engl J Med 2002;346:393-403.

47 Ramachandran A, Snehalatha C, Mary S, Mukesh B, Bhaskar AD, Vijay V: The Indian Diabetes Prevention Programme shows that lifestyle modification and metformin prevent type 2 diabetes in Asian Indian subjects with impaired glucose tolerance (IDPP-1). Diabetologia 2006;49:289-297.

48 Pan XR, Li GW, Hu YH, Wang JX, Yang WY, An ZX, Hu ZX, Lin J, Xiao JZ, Cao HB, Liu $P A$, Jiang $X G$, Jiang YY, Wang JP, Zheng $H$, Zhang H, Bennett PH, Howard BV: Effects of diet and exercise in preventing NIDDM in people with impaired glucose tolerance. The Da Qing IGT and Diabetes Study. Diabetes Care 1997;20:537-544

49 Eriksson KF, Lindgärde F: Prevention of type 2 (non-insulin-dependent) diabetes mellitus by diet and physical exercise. The 6-year Malmö feasibility study. Diabetologia 1991;34:891-898.

50 Swinburn BA, Metcalf PA, Ley SJ: Long-term (5-year) effects of a reduced-fat diet intervention in individuals with glucose intolerance. Diabetes Care 2001;24:619-624. 
-51 Tinker LF, Bonds DE, Margolis KL, Manson JE, Howard BV, Larson J, Perri MG, Beresford SA, Robinson JG, Rodríguez B, Safford MM, Wenger NK, Stevens VJ, Parker LM; Women's Health Initiative: Low-fat dietary pattern and risk of treated diabetes mellitus in postmenopausal women: the Women's Health Initiative randomized controlled dietary modification trial. Arch Intern Med 2008;168:1500-1511.

52 Marshall JA, Hoag S, Shetterly S, Hamman RF: Dietary fat predicts conversion from impaired glucose tolerance to NIDDM. The San Luis Valley diabetes study. Diabetes Care 1994;17:50-56.

53 Feskens EJ, Virtanen SM, Räsänen L, Tuomilehto J, Stengård J, Pekkanen J, Nissinen A, Kromhout D: Dietary factors determining diabetes and impaired glucose tolerance. A 20 year follow-up of the Finnish and Dutch cohorts of the seven countries study. Diabetes Care 1995;18:1104-1112.

-54 Salmerón J, Hu FB, Manson JE, Stampfer MJ, Colditz GA, Rimm EB, Willett WC: Dietary fat intake and risk of type 2 diabetes in women. Am J Clin Nutr 2001;73:1019-1026.

55 Halton TL, Liu S, Manson JE, Hu FB: Lowcarbohydrate-diet score and risk of type 2 diabetes in women. Am J Clin Nutr 2008;87: 339-346.

56 Meyer KA, Kushi LH, Jacobs DR Jr, Folsom AR: Dietary fat and incidence of type 2 diabetes in older Iowa women. Diabetes Care 2001; 24:1528-1535.

57 van Dam RM, Willett WC, Rimm EB, Stampfer MJ, Hu FB: Dietary fat and meat intake in relation to risk of type 2 diabetes in men. Diabetes Care 2002;25:417-424.

58 Laaksonen DE, Lakka TA, Lakka HM, Nyyssönen K, Rissanen T, Niskanen LK, Salonen JT: Serum fatty acid composition predicts development of impaired fasting glycaemia and diabetes in middle-aged men. Diabet Med 2002;19:456-464.

59 Harding AH, Day NE, Khaw KT, Bingham S, Luben R, Welsh A, Wareham NJ: Dietary fat and the risk of clinical type 2 diabetes: the European prospective investigation of Cancer-Norfolk study. Am J Epidemiol 2004;159: $73-82$.

-60 Simmons RK, Harding AH, Jakes RW, Welch A, Wareham NJ, Griffin SJ: How much might achievement of diabetes prevention behaviour goals reduce the incidence of diabetes if implemented at the population level? Diabetologia 2006;49:905-911.

-61 Lindström J, Peltonen M, Eriksson JG, Louheranta A, Fogelholm M, Uusitupa M, Tuomilehto J: High-fibre, low-fat diet predicts long-term weight loss and decreased type 2 diabetes risk: the Finnish Diabetes Prevention Study. Diabetologia 2006;49:912-920.

-62 Hodge AM, English DR, O'Dea K, Sinclair AJ, Makrides M, Gibson RA, Giles GG: Plasma phospholipid and dietary fatty acids as predictors of type 2 diabetes: interpreting the role of linoleic acid. Am J Clin Nutr 2007;86:189197.
63 Schulze MB, Schulz M, Heidemann C, Schienkiewitz A, Hoffmann K, Boeing H: Carbohydrate intake and incidence of type 2 diabetes in the European Prospective Investigation into Cancer and Nutrition (EPIC)-Potsdam Study. Br J Nutr 2008;99:1107-1116.

64 Micha R, Mozaffarian D: Saturated fat and cardiometabolic risk factors, coronary heart disease, stroke, and diabetes: a fresh look at the evidence. Lipids 2010;45:893-905.

65 Song Y, Manson JE, Buring JE, Liu S: A prospective study of red meat consumption and type 2 diabetes in middle-aged and elderly women: the women's health study. Diabetes Care 2004;27:2108-2115.

66 Kröger J, Zietemann V, Enzenbach C, Weikert C, Jansen EH, Döring F, Joost HG, Boeing H, Schulze MB: Erythrocyte membrane phospholipid fatty acids, desaturase activity, and dietary fatty acids in relation to risk of type 2 diabetes in the European Prospective Investigation into Cancer and Nutrition (EPIC)Potsdam Study. Am J Clin Nutr 2011;93:127142.

67 Wang L, Folsom AR, Zheng ZJ, Pankow JS, Eckfeldt JH; ARIC Study Investigators: Plasma fatty acid composition and incidence of diabetes in middle-aged adults: the Atherosclerosis Risk in Communities (ARIC) Study. Am J Clin Nutr 2003;78:91-98.

68 Krachler B, Norberg M, Eriksson JW, Hallmans G, Johansson I, Vessby B, Weinehall L, Lindahl B: Fatty acid profile of the erythrocyte membrane preceding development of type 2 diabetes mellitus. Nutr Metab Cardiovasc Dis 2008; 18:503-510.

69 Brostow DP, Odegaard AO, Koh WP, Duval S, Gross MD, Yuan JM, Pereira MA: Omega-3 fatty acids and incident type 2 diabetes: the Singapore Chinese Health Study. Am J Clin Nutr 2011;94:520-526.

$70 \mathrm{Wu} J \mathrm{H}$, Micha R, Imamura F, Pan A, Biggs ML, Ajaz O, Djousse L, Hu FB, Mozaffarian D: Omega- 3 fatty acids and incident type 2 diabetes: a systematic review and meta-analysis. Br J Nutr 2012;107(suppl 2):S214-S227.

71 Xun P, He K: Fish consumption and incidence of diabetes: meta-analysis of data from 438,000 individuals in 12 independent prospective cohorts with an average 11-year follow-up. Diabetes Care 2012;35:930-938.

72 Wallin A, Di Giuseppe D, Orsini N, Patel PS, Forouhi NG, Wolk A: Fish consumption, dietary long-chain n-3 fatty acids, and risk of type 2 diabetes: systematic review and metaanalysis of prospective studies. Diabetes Care 2012;35:918-929.

73 Mozaffarian D, Cao H, King IB, Lemaitre RN, Song X, Siscovick DS, Hotamisligil GS: Trans-palmitoleic acid, metabolic risk factors, and new-onset diabetes in U.S. adults: a cohort study. Ann Intern Med 2010;153: 790-799.

74 Forouhi NG, Koulman A, Sharp SJ, Imamura F, Kröger J, Schulze MB, Crowe FL, Huerta JM, Guevara M, Beulens JW, van Woudenbergh GJ, Wang L, Summerhill K, Griffin JL,
Feskens EJ, Amiano P, Boeing H, ClavelChapelon F, Dartois L, Fagherazzi G, Franks PW, Gonzalez C, Jakobsen MU, Kaaks R, Key TJ, Khaw KT, Kühn T, Mattiello A, Nilsson PM, Overvad K, Pala V, Palli D, Quirós JR, Rolandsson O, Roswall N, Sacerdote C, Sánchez MJ, Slimani N, Spijkerman AM, Tjonneland A, Tormo MJ, Tumino R, van der A DL, van der Schouw YT, Langenberg C, Riboli E, Wareham NJ: Differences in the prospective association between individual plasma phospholipid saturated fatty acids and incident type 2 diabetes: the EPIC-InterAct casecohort study. Lancet Diabetes Endocrinol 2014;2:810-818.

75 Aune D, Norat T, Romundstad P, Vatten LJ: Dairy products and the risk of type 2 diabetes: a systematic review and dose-response metaanalysis of cohort studies. Am J Clin Nutr 2013;98:1066-1083.

-76 Nicklas TA, Dwyer J, Feldman HA, Luepker RV, Kelder SH, Nader PR: Serum cholesterol levels in children are associated with dietary fat and fatty acid intake. J Am Diet Assoc 2002;102:511-517.

77 Lefevre M, Champagne CM, Tulley RT, Rood JC, Most MM: Individual variability in cardiovascular disease risk factor responses to low-fat and low-saturated-fat diets in men: body mass index, adiposity, and insulin resistance predict changes in LDL cholesterol. Am J Clin Nutr 2005;82:957-963; quiz 11451146.

78 Ginsberg HN, Kris-Etherton P, Dennis B, Elmer PJ, Ershow A, Lefevre M, Pearson T, Roheim P, Ramakrishnan R, Reed R, Stewart K, Stewart P, Phillips K, Anderson N: Effects of reducing dietary saturated fatty acids on plasma lipids and lipoproteins in healthy subjects: the DELTA Study, protocol 1. Arterioscler Thromb Vasc Biol 1998; 18:441449.

79 Mensink RP, Katan MB: Effect of dietary fatty acids on serum lipids and lipoproteins. A meta-analysis of 27 trials. Arterioscler Thromb 1992;12:911-919.

80 Mensink RP, Zock PL, Kester AD, Katan MB: Effects of dietary fatty acids and carbohydrates on the ratio of serum total to HDL cholesterol and on serum lipids and apolipoproteins: a meta-analysis of 60 controlled trials. Am J Clin Nutr 2003;77:1146-1155.

81 Bonanome A, Grundy SM: Effect of dietary stearic acid on plasma cholesterol and lipoprotein levels. N Engl J Med 1988;318:1244-1248.

82 Effect of dietary stearic acid on plasma cholesterol level. N Engl J Med 1988;319:1089-1091.

83 Howell WH, McNamara DJ, Tosca MA, Smith BT, Gaines JA: Plasma lipid and lipoprotein responses to dietary fat and cholesterol: a meta-analysis. Am J Clin Nutr 1997; 65:1747-1764.

84 Sarkkinen ES, Uusitupa MI, Pietinen P, Aro A, Ahola I, Penttilä I, Kervinen K, Kesäniemi YA: Long-term effects of three fat-modified diets in hypercholesterolemic subjects. Atherosclerosis 1994;105:9-23. 
$\$ 85$ Due A, Larsen TM, Mu H, Hermansen K, Stender S, Astrup A: Comparison of 3 ad libitum diets for weight-loss maintenance, risk of cardiovascular disease, and diabetes: a 6-mo randomized, controlled trial. Am J Clin Nutr 2008;88:1232-1241.

-86 Niinikoski H, Lagström H, Jokinen E, Siltala M, Rönnemaa T, Viikari J, Raitakari OT, Jula A, Marniemi J, Näntö-Salonen $\mathrm{K}$, Simell $\mathrm{O}$ : Impact of repeated dietary counseling between infancy and 14 years of age on dietary intakes and serum lipids and lipoproteins: the STRIP study. Circulation 2007;116:1032-1040.

-87 Niinikoski H, Pahkala K, Ala-Korpela M, Viikari J, Rönnemaa T, Lagström H, Jokinen E, Jula A, Savolainen MJ, Simell O, Raitakari OT: Effect of repeated dietary counseling on serum lipoproteins from infancy to adulthood. Pediatrics 2012;129:e704-e713.

-88 Müller H, Lindman AS, Brantsaeter AL, Pedersen JI: The serum LDL/HDL cholesterol ratio is influenced more favorably by exchanging saturated with unsaturated fat than by reducing saturated fat in the diet of women. J Nutr 2003;133:78-83.

-89 Bos MB, de Vries JH, Feskens EJ, van Dijk SJ, Hoelen DW, Siebelink E, Heijligenberg R, de Groot LC: Effect of a high monounsaturated fatty acids diet and a Mediterranean diet on serum lipids and insulin sensitivity in adults with mild abdominal obesity. Nutr Metab Cardiovasc Dis 2010;20:591-598.

90 Allman-Farinelli MA, Gomes K, Favaloro EJ, Petocz P: A diet rich in high-oleic-acid sunflower oil favorably alters low-density lipoprotein cholesterol, triglycerides, and factor VII coagulant activity. J Am Diet Assoc 2005; 105:1071-1079.

-91 Gardner CD, Kraemer HC: Monounsaturated versus polyunsaturated dietary fat and serum lipids. A meta-analysis. Arterioscler Thromb Vasc Biol 1995;15:1917-1927.

-92 Wien MA, Sabaté JM, Iklé DN, Cole SE, Kandeel FR: Almonds vs complex carbohydrates in a weight reduction program. Int J Obes Relat Metab Disord 2003;27:1365-1372.

-93 Novotny JA, Gebauer SK, Baer DJ: Discrepancy between the Atwater factor predicted and empirically measured energy values of almonds in human diets. Am J Clin Nutr 2012;96:296-301.

-94 Shai I, Schwarzfuchs D, Henkin Y, Shahar DR, Witkow S, Greenberg I, Golan R, Fraser D, Bolotin A, Vardi H, Tangi-Rozental O, Zuk-Ramot R, Sarusi B, Brickner D, Schwartz Z, Sheiner E, Marko R, Katorza E, Thiery J, Fiedler GM, Bluher M, Stumvoll M, Stampfer MJ: Weight loss with a low-carbohydrate, Mediterranean, or low-fat diet. N Engl J Med 2008;359:229-241.

95 Thijssen MA, Mensink RP: Small differences in the effects of stearic acid, oleic acid, and linoleic acid on the serum lipoprotein profile of humans. Am J Clin Nutr 2005;82:510-516.

96 Mensink RP, Katan MB: Effect of monounsaturated fatty acids versus complex carbohydrates on high-density lipoproteins in healthy men and women. Lancet 1987;1:122-125.
-97 Egert S, Kannenberg F, Somoza V, Erbersdobler HF, Wahrburg U: Dietary alpha-linolenic acid, EPA, and DHA have differential effects on LDL fatty acid composition but similar effects on serum lipid profiles in normolipidemic humans. J Nutr 2009;139:861868.

$\$ 98$ Karvonen HM, Tapola NS, Uusitupa MI, Sarkkinen ES: The effect of vegetable oilbased cheese on serum total and lipoprotein lipids. Eur J Clin Nutr 2002;56:1094-1101.

-99 Sanders TA, Roshanai F: The influence of different types of omega 3 polyunsaturated fatty acids on blood lipids and platelet function in healthy volunteers. Clin Sci (Lond) 1983;64:91-99.

100 Pang D, lman-Farinelli MA, Wong T, Barnes R, Kingham KM: Replacement of linoleic acid with alpha-linolenic acid does not alter blood lipids in normolipidaemic men. $\mathrm{Br} \mathrm{J}$ Nutr 1998;80:163-167.

101 Finnegan YE, Minihane AM, Leigh-Firbank EC, Kew S, Meijer GW, Muggli R, Calder PC, Williams CM: Plant- and marine-derived $n-3$ polyunsaturated fatty acids have differential effects on fasting and postprandial blood lipid concentrations and on the susceptibility of LDL to oxidative modification in moderately hyperlipidemic subjects. Am J Clin Nutr 2003;77:783-795.

-102 Singer P, Berger I, Wirth M, Gödicke W, Jaeger W, Voigt S: Slow desaturation and elongation of linoleic and alpha-linolenic acids as a rationale of eicosapentaenoic acid-rich diet to lower blood pressure and serum lipids in normal, hypertensive and hyperlipemic subjects. Prostaglandins Leukot Med 1986;24:173-193.

103 Bemelmans WJ, Broer J, Feskens EJ, Smit AJ, Muskiet FA, Lefrandt JD, Bom VJ, May JF, Meyboom-de Jong B: Effect of an increased intake of alpha-linolenic acid and group nutritional education on cardiovascular risk factors: the Mediterranean Alpha-Linolenic Enriched Groningen Dietary Intervention (MARGARIN) study. Am J Clin Nutr 2002; 75:221-227.

104 Schaefer EJ, Lichtenstein AH, Lamon-Fava S, Contois JH, Li Z, Goldin BR, Rasmussen H, McNamara JR, Ordovas JM: Effects of National Cholesterol Education Program Step 2 diets relatively high or relatively low in fish-derived fatty acids on plasma lipoproteins in middle-aged and elderly subjects. Am J Clin Nutr 1996;63:234-241.

105 Watanabe N, Watanabe Y, Kumagai M, Fujimoto K: Administration of dietary fish oil capsules in healthy middle-aged Japanese men with a high level of fish consumption. Int J Food Sci Nutr 2009;60(suppl 5):136-142.

106 García-Alonso FJ, Jorge-Vidal V, Ros G, Periago MJ: Effect of consumption of tomato juice enriched with n-3 polyunsaturated fatty acids on the lipid profile, antioxidant biomarker status, and cardiovascular disease risk in healthy women. Eur J Nutr 2012;51: 415-424.
07 Cazzola R, Russo-Volpe S, Miles EA, Rees D, Banerjee T, Roynette CE, Wells SJ, Goua M, Wahle KW, Calder PC, Cestaro B: Age- and dose-dependent effects of an eicosapentaenoic acid-rich oil on cardiovascular risk factors in healthy male subjects. Atherosclerosis 2007;193:159-167.

108 Damsgaard CT, Frøkiaer H, Andersen AD, Lauritzen L: Fish oil in combination with high or low intakes of linoleic acid lowers plasma triacylglycerols but does not affect other cardiovascular risk markers in healthy men. J Nutr 2008;138:1061-1066.

109 Fontani G, Corradeschi F, Felici A, Alfatti F, Bugarini R, Fiaschi AI, Cerretani D, Montorfano G, Rizzo AM, Berra B: Blood profiles, body fat and mood state in healthy subjects on different diets supplemented with omega-3 polyunsaturated fatty acids. Eur J Clin Invest 2005;35:499-507.

110 Kaul N, Kreml R, Austria JA, Richard MN, Edel AL, Dibrov E, Hirono S, Zettler ME, Pierce GN: A comparison of fish oil, flaxseed oil and hempseed oil supplementation on selected parameters of cardiovascular health in healthy volunteers. J Am Coll Nutr 2008;27: 51-58.

111 Bernstein AM, Ding EL, Willett WC, Rimm EB: A meta-analysis shows that docosahexaenoic acid from algal oil reduces serum triglycerides and increases HDL-cholesterol and LDL-cholesterol in persons without coronary heart disease. J Nutr 2012;142:99104.

112 Milte CM, Coates AM, Buckley JD, Hill AM, Howe PR: Dose-dependent effects of docosahexaenoic acid-rich fish oil on erythrocyte docosahexaenoic acid and blood lipid levels. Br J Nutr 2008;99:1083-1088.

113 Caslake MJ, Miles EA, Kofler BM, Lietz G, Curtis P, Armah CK, Kimber AC, Grew JP, Farrell L, Stannard J, Napper FL, Sala-Vila A, West AL, Mathers JC, Packard C, Williams CM, Calder PC, Minihane AM: Effect of sex and genotype on cardiovascular biomarker response to fish oils: the FINGEN Study. Am J Clin Nutr 2008;88:618-629.

114 Zock PL, Katan MB: Hydrogenation alternatives: effects of trans fatty acids and stearic acid versus linoleic acid on serum lipids and lipoproteins in humans. J Lipid Res 1992;33: 399-410.

115 Mozaffarian D, Clarke R: Quantitative effects on cardiovascular risk factors and coronary heart disease risk of replacing partially hydrogenated vegetable oils with other fats and oils. Eur J Clin Nutr 2009;63(suppl 2): S22-S33.

116 Wanders AJ, Brouwer IA, Siebelink E, Katan MB: Effect of a high intake of conjugated linoleic acid on lipoprotein levels in healthy human subjects. PLoS One 2010;5:e9000.

117 Sundram K, Karupaiah T, Hayes KC: Stearic acid-rich interesterified fat and trans-rich fat raise the $\mathrm{LDL} / \mathrm{HDL}$ ratio and plasma glucose relative to palm olein in humans. Nutr Metab (Lond) 2007;4:3. 
118 Tholstrup T, Raff M, Basu S, Nonboe P, Sejrsen K, Straarup EM: Effects of butter high in ruminant trans and monounsaturated fatty acids on lipoproteins, incorporation of fatty acids into lipid classes, plasma C-reactive protein, oxidative stress, hemostatic variables, and insulin in healthy young men. Am J Clin Nutr 2006;83:237243.

-119 Motard-Bélanger A, Charest A, Grenier G, Paquin P, Chouinard Y, Lemieux S, Couture P, Lamarche B: Study of the effect of trans fatty acids from ruminants on blood lipids and other risk factors for cardiovascular disease. Am J Clin Nutr 2008;87:593-599.

$\$ 120$ Chardigny JM, Destaillats F, MalpuechBrugère $C$, Moulin J, Bauman DE, Lock $A L$, Barbano DM, Mensink RP, Bezelgues JB, Chaumont P, Combe N, Cristiani I, Joffre F, German JB, Dionisi F, Boirie Y, Sébédio JL: Do trans fatty acids from industrially produced sources and from natural sources have the same effect on cardiovascular disease risk factors in healthy subjects? Results of the trans Fatty Acids Collaboration (TRANSFACT) study. Am J Clin Nutr 2008; 87:558-566.

121 Sluijs I, Plantinga Y, de Roos B, Mennen LI, Bots ML: Dietary supplementation with cis9,trans-11 conjugated linoleic acid and aortic stiffness in overweight and obese adults. Am J Clin Nutr 2010;91:175-183.

$\checkmark 122$ Lambert EV, Goedecke JH, Bluett K, Heggie K, Claassen A, Rae DE, West S, Dugas J, Dugas L, Meltzeri S, Charlton K, Mohede I: Conjugated linoleic acid versus high-oleic acid sunflower oil: effects on energy metabolism, glucose tolerance, blood lipids, appetite and body composition in regularly exercising individuals. Br J Nutr 2007;97:1001-1011.

123 Mistry P, Miller NE, Laker M, Hazzard WR, Lewis B: Individual variation in the effects of dietary cholesterol on plasma lipoproteins and cellular cholesterol homeostasis in man. Studies of low density lipoprotein receptor activity and 3-hydroxy-3-methylglutaryl coenzyme A reductase activity in blood mononuclear cells. J Clin Invest 1981;67:493-502.

124 Katan MB, Beynen AC: Hyper-response to dietary cholesterol in man. Lancet 1983;1: 1213.

125 Beynen AC, Katan MB, van Zutphen BFM: Individuelle Unterschiede der Serumcholesterinreaktion auf Änderungen der Ernährungsform. Ernährungs Umschau 1985;32:356-360.

126 Wolff E, Vergnes ME, Portugal H, Defoort C, Amiot-Carlin MJ, Lairon D, Nicolay A: Cholesterol-absorber status modifies the LDL cholesterol-lowering effect of a Mediterranean-type diet in adults with moderate cardiovascular risk factors. J Nutr 2011;141: 1791-1798.

127 Clarke R, Frost C, Collins R, Appleby P, Peto $\mathrm{R}$ : Dietary lipids and blood cholesterol: quantitative meta-analysis of metabolic ward studies. BMJ 1997;314:112-117.
128 Weggemans RM, Zock PL, Katan MB: Dietary cholesterol from eggs increases the ratio of total cholesterol to high-density lipoprotein cholesterol in humans: a meta-analysis. Am J Clin Nutr 2001;73:885-891.

129 Hopkins PN: Effects of dietary cholesterol on serum cholesterol: a meta-analysis and review. Am J Clin Nutr 1992;55:1060-1070.

$\checkmark 130$ Fielding CJ, Havel RJ, Todd KM, Yeo KE, Schloetter MC, Weinberg V, Frost PH: Effects of dietary cholesterol and fat saturation on plasma lipoproteins in an ethnically diverse population of healthy young men. J Clin Invest 1995;95:611-618.

131 Greene CM, Zern TL, Wood RJ, Shrestha S, Aggarwal D, Sharman MJ, Volek JS, Fernandez ML: Maintenance of the LDL cholesterol: HDL cholesterol ratio in an elderly population given a dietary cholesterol challenge. J Nutr 2005; 135:2793-2798.

132 Greene CM, Waters D, Clark RM, Contois JH, Fernandez ML: Plasma LDL and HDL characteristics and carotenoid content are positively influenced by egg consumption in an elderly population. Nutr Metab (Lond) 2006;3:6.

133 Hjerpsted J, Leedo E, Tholstrup T: Cheese intake in large amounts lowers LDL-cholesterol concentrations compared with butter intake of equal fat content. Am J Clin Nutr 2011;94:1479-1484.

134 Bjermo H, Iggman D, Kullberg J, Dahlman I, Johansson L, Persson L, Berglund J, Pulkki K, Basu S, Uusitupa M, Rudling M, Arner P, Cederholm T, Ahlström H, Risérus U: Effects of n-6 PUFAs compared with SFAs on liver fat, lipoproteins, and inflammation in abdominal obesity: a randomized controlled trial. Am J Clin Nutr 2012;95:1003-1012.

135 Bansal S, Buring JE, Rifai N, Mora S, Sacks FM, Ridker PM: Fasting compared with nonfasting triglycerides and risk of cardiovascular events in women. JAMA 2007;298: 309-316.

136 Nordestgaard BG, Benn M, Schnohr P, Tybjaerg-Hansen A: Nonfasting triglycerides and risk of myocardial infarction, ischemic heart disease, and death in men and women. JAMA 2007;298:299-308.

137 Mora S, Rifai N, Buring JE, Ridker PM: Fasting compared with nonfasting lipids and apolipoproteins for predicting incident cardiovascular events. Circulation 2008;118: 993-1001.

-138 Varbo A, Benn M, Tybjærg-Hansen A, Jørgensen AB, Frikke-Schmidt R, Nordestgaard BG: Remnant cholesterol as a causal risk factor for ischemic heart disease. J Am Coll Cardiol 2013;61:427-436.

139 Varbo A, Benn M, Nordestgaard BG: Remnant cholesterol as a cause of ischemic heart disease: evidence, definition, measurement, atherogenicity, high risk patients, and present and future treatment. Pharmacol Ther 2014;141:358-367.

140 Howard BV, van Horn L, Hsia J, Manson JE, Stefanick ML, Wassertheil-Smoller S, Kuller
LH, LaCroix AZ, Langer RD, Lasser NL, Lewis CE, Limacher MC, Margolis KL, Mysiw WJ, Ockene JK, Parker LM, Perri MG, Phillips L, Prentice RL, Robbins J, Rossouw JE, Sarto GE, Schatz IJ, Snetselaar LG, Stevens VJ, Tinker LF, Trevisan M, Vitolins MZ, Anderson GL, Assaf AR, Bassford T, Beresford SA, Black HR, Brunner RL, Brzyski RG Caan B, Chlebowski RT, Gass M, Granek I, Greenland P, Hays J, Heber D, Heiss G, Hendrix SL, Hubbell FA, Johnson KC, Kotchen JM: Low-fat dietary pattern and risk of cardiovascular disease: the women's health initiative randomized controlled dietary modification trial. JAMA 2006;295:655-666.

141 Nordmann AJ, Nordmann A, Briel M, Keller U, Yancy WS Jr, Brehm BJ, Bucher HC: Effects of low-carbohydrate vs low-fat diets on weight loss and cardiovascular risk factors: a meta-analysis of randomized controlled trials. Arch Intern Med 2006;166:285-293.

$142 \mathrm{Hu}$ T, Mills KT, Yao L, Demanelis K, Eloustaz M, Yancy WS, Kelly TN, He J, Bazzano LA: Effects of low-carbohydrate diets versus low-fat diets on metabolic risk factors: a meta-analysis of randomized controlled clinical trials. Am J Epidemiol 2012; 176(suppl 7):S44-S54.

143 Sacks FM, Bray GA, Carey VJ, Smith SR, Ryan DH, Anton SD, McManus K, Champagne CM, Bishop LM, Laranjo N, Leboff MS, Rood JC, de Jonge L, Greenway FL, Loria $\mathrm{CM}$, Obarzanek E, Williamson DA: Comparison of weight-loss diets with different compositions of fat, protein, and carbohydrates. N Engl J Med 2009;360:859-873.

144 Brussaard JH, van Raaij JM, Stasse-Wolthuis M, Katan MB, Hautvast JG: Blood pressure and diet in normotensive volunteers: absence of an effect of dietary fiber, protein, or fat. Am J Clin Nutr 1981;34:2023-2029.

145 Margetts BM, Beilin LJ, Armstrong BK, Rouse IL, Vandongen R, Croft KD, McMurchie EJ: Blood pressure and dietary polyunsaturated and saturated fats: a controlled trial. Clin Sci (Lond) 1985;69:165-175.

146 Sacks FM, Stampfer MJ, Munoz A, McManus K, Canessa M, Kass EH: Effect of linoleic and oleic acids on blood pressure, blood viscosity, and erythrocyte cation transport. J Am Coll Nutr 1987;6:179-185.

147 Mensink RP, Stolwijk AM, Katan MB: Effect of a monounsaturated diet vs. a polyunsaturated fatty acid-enriched diet on blood pressure in normotensive women and men. Eur J Clin Invest 1990;20:463-469.

148 Zock PL, Blijlevens RA, de Vries JH, Katan MB: Effects of stearic acid and trans fatty acids versus linoleic acid on blood pressure in normotensive women and men. Eur J Clin Nutr 1993;47:437-444.

-149 Aro A, Pietinen P, Valsta LM, Salminen I, Turpeinen AM, Virtanen M, Dougherty RM, Iacono JM: Lack of effect on blood pressure by low fat diets with different fatty acid compositions. J Hum Hypertens 1998; 12: 383-389. 
150 Stamler J, Liu K, Ruth KJ, Pryer J, Greenland P: Eight-year blood pressure change in middle-aged men: relationship to multiple nutrients. Hypertension 2002;39: 1000-1006.

-151 Ascherio A, Rimm EB, Giovannucci EL, Colditz GA, Rosner B, Willett WC, Sacks F, Stampfer MJ: A prospective study of nutritional factors and hypertension among US men. Circulation 1992;86:1475-1484.

152 Wang L, Manson JE, Forman JP, Gaziano JM, Buring JE, Sesso HD: Dietary fatty acids and the risk of hypertension in middle-aged and older women. Hypertension 2010;56: 598-604.

153 Shah M, Adams-Huet B, Garg A: Effect of high-carbohydrate or high-cis-monounsaturated fat diets on blood pressure: a metaanalysis of intervention trials. Am J Clin Nutr 2007;85:1251-1256.

154 Brehm BJ, Lattin BL, Summer SS, Boback JA, Gilchrist GM, Jandacek RJ, D’Alessio DA: One-year comparison of a high-monounsaturated fat diet with a high-carbohydrate diet in type 2 diabetes. Diabetes Care 2009;32: 215-220.

155 Jenkins DJ, Chiavaroli L, Wong JM, Kendall C, Lewis GF, Vidgen E, Connelly PW, Leiter LA, Josse RG, Lamarche B: Adding monounsaturated fatty acids to a dietary portfolio of cholesterol-lowering foods in hypercholesterolemia. CMAJ 2010;182: 1961-1967.

-156 Rasmussen BM, Vessby B, Uusitupa M, Berglund L, Pedersen E, Riccardi G, Rivellese AA, Tapsell L, Hermansen K: Effects of dietary saturated, monounsaturated, and n-3 fatty acids on blood pressure in healthy subjects. Am J Clin Nutr 2006;83:221-226.

-157 Wendland E, Farmer A, Glasziou P, Neil A: Effect of alpha linolenic acid on cardiovascular risk markers: a systematic review. Heart 2006;92:166-169.

158 Paschos GK, Magkos F, Panagiotakos DB, Votteas V, Zampelas A: Dietary supplementation with flaxseed oil lowers blood pressure in dyslipidaemic patients. Eur J Clin Nutr 2007;61:1201-1206.

159 Appel LJ, Miller ER, Seidler AJ, Whelton PK: Does supplementation of diet with 'fish oil' reduce blood pressure? A meta-analysis of controlled clinical trials. Arch Intern Med 1993;153:1429-1438.

160 Morris MC, Sacks F, Rosner B: Does fish oil lower blood pressure? A meta-analysis of controlled trials. Circulation 1993;88:523533.

161 Geleijnse JM, Giltay EJ, Grobbee DE, Donders AR, Kok FJ: Blood pressure response to fish oil supplementation: metaregression analysis of randomized trials. J Hypertens 2002;20:1493-1499.

162 Campbell F, Dickinson HO, Critchley JA, Ford GA, Bradburn M: A systematic review of fish-oil supplements for the prevention and treatment of hypertension. Eur J Prev Cardiol 2013;20:107-120.
163 Miller PE, van Elswyk M, Alexander DD: Long-chain omega-3 fatty acids eicosapentaenoic acid and docosahexaenoic acid and blood pressure: a meta-analysis of randomized controlled trials. Am J Hypertens 2014; 27:885-896

164 Xun P, Hou N, Daviglus M, Liu K, Morris JS, Shikany JM, Sidney S, Jacobs DR, He K: Fish oil, selenium and mercury in relation to incidence of hypertension: a 20-year follow-up study. J Intern Med 2011;270:175-186.

165 Expert Panel on Detection, Evaluation, and Treatment of High Blood Cholesterol in Adults: Executive summary of the third report of the national cholesterol education program (NCEP) expert panel on detection, evaluation, and treatment of high blood cholesterol in adults (adult treatment panel III). JAMA 2001;285:2486-2497.

166 Alberti KG, Zimmet P, Shaw J: The metabolic syndrome - a new worldwide definition. Lancet 2005;366:1059-1062.

-167 Dorgan JF, Liu L, Barton BA, Deshmukh S, Snetselaar LG, van Horn L, Stevens VJ, Robson AM, Lasser NL, Himes JH, Shepherd JA, Pourfarzib R, Pettee Gabriel K, Kriska A, Kwiterovich PO Jr: Adolescent diet and metabolic syndrome in young women: results of the Dietary Intervention Study in Children (DISC) follow-up study. J Clin Endocrinol Metab 2011;96:E1999-E2008.

168 Ilanne-Parikka P, Eriksson JG, Lindström J, Peltonen $M$, Aunola $S$, Hämäläinen $H$, Keinänen-Kiukaanniemi S, Laakso M, Valle TT, Lahtela J, Uusitupa M, Tuomilehto J: Effect of lifestyle intervention on the occurrence of metabolic syndrome and its components in the Finnish Diabetes Prevention Study. Diabetes Care 2008;31:805-807.

169 Bo S, Ciccone G, Baldi C, Benini L, Dusio F, Forastiere G, Lucia C, Nuti C, Durazzo M, Cassader M, Gentile L, Pagano G: Effectiveness of a lifestyle intervention on metabolic syndrome. A randomized controlled trial. J Gen Intern Med 2007;22:1695-1703.

170 Johnson JL, Slentz CA, Houmard JA, Samsa GP, Duscha BD, Aiken LB, McCartney JS, Tanner CJ, Kraus WE: Exercise training amount and intensity effects on metabolic syndrome (from studies of a targeted risk reduction intervention through defined exercise). Am J Cardiol 2007;100:1759-1766.

171 Camhi SM, Stefanick ML, Katzmarzyk PT, Young DR: Metabolic syndrome and changes in body fat from a low-fat diet and/or exercise randomized controlled trial. Obesity 2010;18:548-554.

172 Carnethon MR, Loria CM, Hill JO, Sidney S, Savage PJ, Liu K: Risk factors for the metabolic syndrome: the Coronary Artery Risk Development in Young Adults (CARDIA) study, 1985-2001. Diabetes Care 2004;27: 2707-2715.

173 Millen BE, Pencina MJ, Kimokoti RW, Zhu L, Meigs JB, Ordovas JM, D’Agostino RB: Nutritional risk and the metabolic syndrome in women: opportunities for preventive in- tervention from the Framingham Nutrition Study. Am J Clin Nutr 2006;84:434-441.

174 Mirmiran P, Noori N, Azizi F: A prospective study of determinants of the metabolic syndrome in adults. Nutr Metab Cardiovasc Dis 2008;18:567-573.

175 Ventura AK, Loken E, Birch LL: Risk profiles for metabolic syndrome in a nonclinical sample of adolescent girls. Pediatrics 2006; 118:2434-2442.

176 Salas-Salvadó J, Fernandez-Ballart J, Ros E, Martinez-Gonzalez MA, Fito M, Estruch R, Corella D, Fiol M, Gomez-Gracia E, Aros F, Flores G, Lapetra J, Lamuela-Raventos R, Ruiz-Gutierrez V, Bulló M, Basora J, Covas MI: Effect of a Mediterranean diet supplemented with nuts on metabolic syndrome status: one-year results of the PREDIMED randomized trial. Arch Intern Med 2008; 168:2449-2458.

-177 Vanhala M, Saltevo J, Soininen P, Kautiainen H, Kangas AJ, Ala-Korpela M, Mäntyselkä P: Serum omega-6 polyunsaturated fatty acids and the metabolic syndrome: a longitudinal population-based cohort study. Am J Epidemiol 2012;176:253-260.

178 Baik I, Abbott RD, Curb JD, Shin C: Intake of fish and n-3 fatty acids and future risk of metabolic syndrome. J Am Diet Assoc 2010; 110:1018-1026.

179 Warensjö E, Sundström J, Lind L, Vessby B: Factor analysis of fatty acids in serum lipids as a measure of dietary fat quality in relation to the metabolic syndrome in men. Am J Clin Nutr 2006;84:442-448.

180 Kiess W, Hauner H, Wabitsch M, Reinehr T (eds): Das Metabolische Syndrom im Kindes- und Jugendalter: Diagnose - Therapie - Prävention. München, Elsevier, Urban \& Fischer, 2009.

181 Mente A, de Koning L, Shannon HS, Anand SS: A systematic review of the evidence supporting a causal link between dietary factors and coronary heart disease. Arch Intern Med 2009;169:659-669.

182 Skeaff CM, Miller J: Dietary fat and coronary heart disease: summary of evidence from prospective cohort and randomised controlled trials. Ann Nutr Metab 2009;55:173-201.

183 Mozaffarian D, Micha R, Wallace S: Effects on coronary heart disease of increasing polyunsaturated fat in place of saturated fat: a systematic review and meta-analysis of randomized controlled trials. PLoS Med 2010;7:e1000252.

184 Siri-Tarino PW, Sun Q, Hu FB, Krauss RM: Meta-analysis of prospective cohort studies evaluating the association of saturated fat with cardiovascular disease. Am J Clin Nutr 2010;91:535-546.

185 Jakobsen MU, O’Reilly EJ, Heitmann BL, Pereira MA, Bälter K, Fraser GE, Goldbourt U, Hallmans G, Knekt P, Liu S, Pietinen P, Spiegelman D, Stevens J, Virtamo J, Willett WC, Ascherio A: Major types of dietary fat and risk of coronary heart disease: a pooled analysis of 11 cohort studies. Am J Clin Nutr 2009;89:1425-1432. 
186 Jakobsen MU, Dethlefsen C, Joensen AM, $>197$ Mozaffarian D, Rimm EB: Fish intake, conStegger J, Tjønneland A, Schmidt EB, Overvad K: Intake of carbohydrates compared with intake of saturated fatty acids and risk of myocardial infarction: importance of the glycemic index. Am J Clin Nutr 2010;91: 1764-1768.

-187 Chowdhury R, Warnakula S, Kunutsor S, Crowe F, Ward HA, Johnson L, Franco OH, Butterworth AS, Forouhi NG, Thompson SG, Khaw KT, Mozaffarian D, Danesh J, Di Angelantonio E: Association of dietary, circulating, and supplement fatty acids with coronary risk: a systematic review and metaanalysis. Ann Intern Med 2014;160:398-406.

188 Dawczynski C, Kleber ME, März W, Jahreis G, Lorkowski S: Association of dietary, circulating, and supplement fatty acids with coronary risk. Ann Intern Med 2014;161: 453-454.

189 Willett WC, Stampfer MJ, Sacks FM: Association of dietary, circulating, and supplement fatty acids with coronary risk. Ann Intern Med 2014;161:458.

190 Schwingshackl L, Hoffmann G: Monounsaturated fatty acids, olive oil and health status: a systematic review and meta-analysis of cohort studies. Lipids Health Dis 2014;13:154.

-191 Ramsden CE, Hibbeln JR, Majchrzak SF, Davis JM: n-6 fatty acid-specific and mixed polyunsaturate dietary interventions have different effects on CHD risk: a meta-analysis of randomised controlled trials. Br J Nutr 2010;104:1586-1600.

192 de Goede J, Verschuren WM, Boer JM, Verberne LD, Kromhout D, Geleijnse JM: N-6 and N-3 fatty acid cholesteryl esters in relation to fatal CHD in a dutch adult population: a nested case-control study and metaanalysis. PLoS One 2013;8:e59408.

-193 Farvid MS, Ding M, Pan A, Sun Q, Chiuve SE, Steffen LM, Willett WC, Hu FB: Dietary linoleic acid and risk of coronary heart disease: a systematic review and meta-analysis of prospective cohort studies. Circulation 2014;130:1568-1578.

194 de Lorgeril M, Salen P: Dietary prevention of coronary heart disease: focus on omega-6/ omega-3 essential fatty acid balance; in Simopoulos AP, Cleland LG (eds): Omega-6, Omega-3 Essential Fatty Acid Ratio: The Scientific Evidence. World Review of Nutrition and Dietetics. Basel, Karger, 2003, pp $57-73$.

195 Wolfram G: Was sind und wie wirken $\omega-3$ Fettsäuren? Struktur, Stoffwechsel und Wirkungsmechanismen von $\omega$-3-Fettsäuren in der Prävention kardiovaskulärer Krankheiten. Ernährungs Umschau 1997; 44:S36-S41.

196 Natvig H, Borchgrevink CF, Dedichen J, Owren PA, Schiotz EH, Westlund K: A controlled trial of the effect of linolenic acid on incidence of coronary heart disease. The Norwegian vegetable oil experiment of 1965-66. Scand J Clin Lab Invest Suppl 1968; 105:1-20. taminants, and human health: evaluating the risks and the benefits. JAMA 2006;296: 1885-1899.

198 Turpeinen O: Effect of cholesterol-lowering diet on mortality from coronary heart disease and other causes. Circulation 1979;59: $1-7$.

199 Miettinen M, Turpeinen O, Karvonen MJ, Pekkarinen M, Paavilainen E, Elosuo R: Dietary prevention of coronary heart disease in women: the Finnish mental hospital study. Int J Epidemiol 1983;12:17-25.

200 Brouwer IA, Katan MB, Zock PL: Dietary a-linolenic acid is associated with reduced risk of fatal coronary heart disease, but increased prostate cancer risk: a meta-analysis. J Nutr 2004;134:919-922.

201 Pan A, Chen M, Chowdhury R, Wu JH, Sun Q, Campos H, Mozaffarian D: $\alpha$-Linolenic acid and risk of cardiovascular disease: a systematic review and meta-analysis. Am J Clin Nutr 2012;96:1262-1273.

202 Studer M, Briel M, Leimenstoll B, Glass TR, Bucher HC: Effect of different antilipidemic agents and diets on mortality: a systematic review. Arch Intern Med 2005;165:725-730.

203 Hooper L, Thompson RL, Harrison RA, Summerbell CD, Ness AR, Moore HJ, Worthington HV, Durrington PN, Higgins JP, Capps NE, Riemersma RA, Ebrahim SB, Davey Smith G: Risks and benefits of omega 3 fats for mortality, cardiovascular disease, and cancer: systematic review. BMJ 2006; 332:752-760.

204 Brox J, Olaussen K, Osterud B, Elvevoll EO, Bjørnstad E, Brattebøg G, Iversen H: A longterm seal- and cod-liver-oil supplementation in hypercholesterolemic subjects. Lipids 2001;36:7-13.

205 Malaguarnera M, Restuccia N, Fazio ID, Panebianco MP, Gulizia G, Giugno I: Fish oil treatment of interferon-alpha-induced dyslipidaemia: study in patients with chronic hepatitis C. BioDrugs 1999;11:285-291.

206 Yokoyama M, Origasa H, Matsuzaki M, Matsuzawa Y, Saito Y, Ishikawa Y, Oikawa S, Sasaki J, Hishida H, Itakura H, Kita T, Kitabatake A, Nakaya N, Sakata T, Shimada K, Shirato K: Effects of eicosapentaenoic acid on major coronary events in hypercholesterolaemic patients (JELIS): a randomised open-label, blinded endpoint analysis. Lancet 2007;369:1090-1098.

207 Delgado-Lista J, Perez-Martinez P, LopezMiranda J, Perez-Jimenez F: Long chain omega- 3 fatty acids and cardiovascular disease: a systematic review. Br J Nutr 2012; 107(suppl 2):S201-S213.

208 Einvik G, Klemsdal TO, Sandvik L, Hjerkinn EM: A randomized clinical trial on $n-3$ polyunsaturated fatty acids supplementation and all-cause mortality in elderly men at high cardiovascular risk. Eur J Cardiovasc Prev Rehabil 2010;17:588-592.

209 Kotwal S, Jun M, Sullivan D, Perkovic V, Neal B: Omega 3 fatty acids and cardiovas- cular outcomes: systematic review and meta-analysis. Circ Cardiovasc Qual Outcomes 2012;5:808-818.

210 ORIGIN Trial Investigators, Bosch J, Gerstein HC, Dagenais GR, Díaz R, Dyal L, Jung H, Maggiono AP, Probstfield J, Ramachandran A, Riddle MC, Rydén LE, Yusuf S: n-3 fatty acids and cardiovascular outcomes in patients with dysglycemia. N Engl J Med 2012;367:309-318.

211 Musa-Veloso K, Binns MA, Kocenas A, Chung C, Rice H, Oppedal-Olsen H, Lloyd $\mathrm{H}$, Lemke S: Impact of low v. moderate intakes of long-chain n-3 fatty acids on risk of coronary heart disease. Br J Nutr 2011;106: 1129-1141.

212 Trikalinos TA, Lee J, Moorthy D, Yu WW, Lau J, Lichtenstein AH, Chung M: Effects of Eicosapentanoic Acid and Docosahexanoic Acid on Mortality Across Diverse Settings: Systematic Review and Meta-Analysis of Randomized Trials and Prospective Cohorts. Nutritional Research Series, vol. 4. AHRQ Technical Reviews. Rockville, Agency for Healthcare Research and Quality, 2012.

213 Rissanen T, Voutilainen S, Nyyssönen K, Lakka TA, Salonen JT: Fish oil-derived fatty acids, docosahexaenoic acid and docosapentaenoic acid, and the risk of acute coronary events: the Kuopio ischaemic heart disease risk factor study. Circulation 2000;102: 2677-2679.

214 Albert CM, Campos H, Stampfer MJ, Ridker PM, Manson JE, Willett WC, Ma J: Blood levels of long-chain n-3 fatty acids and the risk of sudden death. N Engl J Med 2002;346: 1113-1118.

-215 Lemaitre RN, King IB, Mozaffarian D, Kuller LH, Tracy RP, Siscovick DS: n-3 polyunsaturated fatty acids, fatal ischemic heart disease, and nonfatal myocardial infarction in older adults: the cardiovascular health study. Am J Clin Nutr 2003;77:319-325.

216 Woodward M, Tunstall-Pedoe H, Batty GD, Tavendale R, Hu FB, Czernichow S: The prognostic value of adipose tissue fatty acids for incident cardiovascular disease: results from 3,944 subjects in the Scottish Heart Health Extended Cohort Study. Eur Heart J 2011;32:1416-1423.

217 Mozaffarian D, Lemaitre RN, King IB, Song X, Huang H, Sacks FM, Rimm EB, Wang M, Siscovick DS: Plasma phospholipid longchain $\omega-3$ fatty acids and total and causespecific mortality in older adults: a cohort study. Ann Intern Med 2013;158:515-525.

218 Mozaffarian D, Aro A, Willett WC: Health effects of trans fatty acids: experimental and observational evidence. Eur J Clin Nutr 2009;63(suppl 2):S5-S21.

219 Bendsen NT, Christensen R, Bartels EM, Astrup A: Consumption of industrial and ruminant trans fatty acids and risk of coronary heart disease: a systematic review and metaanalysis of cohort studies. Eur J Clin Nutr 2011;65:773-783. 
220 Shekelle RB, Shryock AM, Paul O, Lepper M, $>233$ de Oliveira Otto MC, Mozaffarian D, Stamler J, Liu S, Raynor WJ Jr: Diet, serum cholesterol, and death from coronary heart disease. The Western Electric study. N Engl J Med 1981;304:65-70.

-221 Shekelle RB, Stamler J: Dietary cholesterol and ischaemic heart disease. Lancet 1989;1: 1177-1179.

222 McGee DL, Reed DM, Yano K, Kagan A, Tillotson J: Ten-year incidence of coronary heart disease in the Honolulu Heart Program. Relationship to nutrient intake. Am J Epidemiol 1984;119:667-676.

223 Kromhout D, Menotti A, Bloemberg B, Aravanis $C$, Blackburn $H$, Buzina $R$, Dontas AS, Fidanza F, Giampaoli S, Jansen A: Dietary saturated and trans fatty acids and cholesterol and 25-year mortality from coronary heart disease: the Seven Countries Study. Prev Med 1995;24:308-315.

224 Stamler J, Shekelle R: Dietary cholesterol and human coronary heart disease. The epidemiologic evidence. Arch Pathol Lab Med 1988;112:1032-1040.

-225 Kushi LH, Lew RA, Stare FJ, Ellison CR, el Lozy M, Bourke G, Daly L, Graham I, Hickey N, Mulcahy R, et al: Diet and 20-year mortality from coronary heart disease. The Ireland-Boston diet-heart study. $\mathrm{N}$ Engl J Med 1985;312:811-818.

-26 Morris JN, Marr JW, Clayton DG: Diet and heart: a postscript. Br Med J 1977;2:13071314.

-227 Kromhout D, de Lezenne Coulander C: Diet, prevalence and 10-year mortality from coronary heart disease in 871 middle-aged men. The Zutphen study. Am J Epidemiol 1984; 119:733-741.

-228 Posner BM, Cobb JL, Belanger AJ, Cupples LA, D'Agostino RB, Stokes J: Dietary lipid predictors of coronary heart disease in men. The Framingham Study. Arch Intern Med 1991;151:1181-1187.

-229 Ascherio A, Rimm EB, Giovannucci EL, Spiegelman D, Stampfer M, Willett WC: Dietary fat and risk of coronary heart disease in men: cohort follow up study in the United States. BMJ 1996;313:84-90.

-230 Esrey KL, Joseph L, Grover SA: Relationship between dietary intake and coronary heart disease mortality: lipid research clinics prevalence follow-up study. J Clin Epidemiol 1996;49:211-216.

-231 Hu FB, Stampfer MJ, Manson JE, Rimm E, Colditz GA, Rosner BA, Hennekens CH, Willett WC: Dietary fat intake and the risk of coronary heart disease in women. N Engl J Med 1997;337:1491-1499.

232 Pietinen P, Ascherio A, Korhonen P, Hartman AM, Willett WC, Albanes D, Virtamo J: Intake of fatty acids and risk of coronary heart disease in a cohort of Finnish men. The Alpha-Tocopherol, Beta-Carotene Cancer Prevention Study. Am J Epidemiol 1997;145: 876-887.
Kromhout D, Bertoni AG, Sibley CT, Jacobs DR Jr, Nettleton JA: Dietary intake of saturated fat by food source and incident cardiovascular disease: the Multi-Ethnic Study of Atherosclerosis. Am J Clin Nutr 2012;96: 397-404.

234 Harris WS: The omega-3 index: clinical utility for therapeutic intervention. Curr Cardiol Rep 2010;12:503-508.

235 McGee D, Reed D, Stemmerman G, Rhoads G, Yano K, Feinleib M: The relationship of dietary fat and cholesterol to mortality in 10 years: the Honolulu Heart Program. Int J Epidemiol 1985;14:97-105.

236 Gillman MW, Cupples LA, Millen BE, Ellison RC, Wolf PA: Inverse association of dietary fat with development of ischemic stroke in men. JAMA 1997;278:2145-2150.

-237 Kagan A, Popper JS, Rhoads GG, Yano K: Dietary and other risk factors for stroke in Hawaiian Japanese men. Stroke 1985;16: 390-396.

238 Reed DM: The paradox of high risk of stroke in populations with low risk of coronary heart disease. Am J Epidemiol 1990;131: 579-588.

239 Seino F, Date C, Nakayama T, Yoshiike N, Yokoyama T, Yamaguchi M, Tanaka H: Dietary lipids and incidence of cerebral infarction in a Japanese rural community. J Nutr Sci Vitaminol 1997;43:83-99.

240 Sauvaget C, Nagano J, Hayashi M, Yamada M: Animal protein, animal fat, and cholesterol intakes and risk of cerebral infarction mortality in the adult health study. Stroke 2004;35:1531-1537.

241 Iso H, Stampfer MJ, Manson JE, Rexrode K, Hu F, Hennekens CH, Colditz GA, Speizer FE, Willett WC: Prospective study of fat and protein intake and risk of intraparenchymal hemorrhage in women. Circulation 2001; 103:856-863.

242 He K, Merchant A, Rimm EB, Rosner BA, Stampfer MJ, Willett WC, Ascherio A: Dietary fat intake and risk of stroke in male US healthcare professionals: 14 year prospective cohort study. BMJ 2003;327:777-782.

243 Boden-Albala B, Elkind MS, White H, Szumski A, Paik MC, Sacco RL: Dietary total fat intake and ischemic stroke risk: the Northern Manhattan Study. Neuroepidemiology 2009;32:296-301.

244 Lapidus L, Andersson H, Bengtsson C, Bosaeus I: Dietary habits in relation to incidence of cardiovascular disease and death in women: a 12-year follow-up of participants in the population study of women in Gothenburg, Sweden. Am J Clin Nutr 1986;44: 444-448.

245 Leosdottir M, Nilsson PM, Nilsson JA, Berglund G: Cardiovascular event risk in relation to dietary fat intake in middle-aged individuals: data from The Malmö Diet and Cancer Study. Eur J Cardiovasc Prev Rehabil 2007; 14:701-706.
246 Larsson SC, Virtamo J, Wolk A: Dietary fats and dietary cholesterol and risk of stroke in women. Atherosclerosis 2012; 221:282-286.

247 Atkinson C, Whitley E, Ness A, Baker I: Associations between types of dietary fat and fish intake and risk of stroke in the Caerphilly Prospective Study (CaPS). Public Health 2011;125:345-348.

248 Iso H, Sato S, Kitamura A, Naito Y, Shimamoto T, Komachi Y: Fat and protein intakes and risk of intraparenchymal hemorrhage among middle-aged Japanese. Am J Epidemiol 2003;157:32-39.

249 Takeya Y, Popper JS, Shimizu Y, Kato H, Rhoads GG, Kagan A: Epidemiologic studies of coronary heart disease and stroke in Japanese men living in Japan, Hawaii and California: incidence of stroke in Japan and Hawaii. Stroke 1984;15:15-23.

250 Yamagishi K, Iso H, Yatsuya H, Tanabe N, Date C, Kikuchi S, Yamamoto A, Inaba Y, Tamakoshi A: Dietary intake of saturated fatty acids and mortality from cardiovascular disease in Japanese: the Japan Collaborative Cohort Study for Evaluation of Cancer Risk (JACC) Study. Am J Clin Nutr 2010;92: 759-765.

251 Misirli G, Benetou V, Lagiou P, Bamia C, Trichopoulos D, Trichopoulou A: Relation of the traditional Mediterranean diet to cerebrovascular disease in a Mediterranean population. Am J Epidemiol 2012;176:11851192.

252 Yaemsiri S, Sen S, Tinker L, Rosamond W, Wassertheil-Smoller S, He K: Trans fat, aspirin, and ischemic stroke in postmenopausal women. Ann Neurol 2012;72:704-715.

253 Chowdhury R, Stevens S, Gorman D, Pan A, Warnakula S, Chowdhury S, Ward H, Johnson L, Crowe F, Hu FB, Franco OH: Association between fish consumption, long chain omega 3 fatty acids, and risk of cerebrovascular disease: systematic review and meta-analysis. BMJ 2012;345:e6698.

254 Larsson SC, Orsini N, Wolk A: Long-chain omega-3 polyunsaturated fatty acids and risk of stroke: a meta-analysis. Eur J Epidemiol 2012;27:895-901.

255 He K: Fish, long-chain omega-3 polyunsaturated fatty acids and prevention of cardiovascular disease - eat fish or take fish oil supplement? Prog Cardiovasc Dis 2009;52:95114.

256 US Department of Agriculture: Composition of foods raw, processed, prepared. USDA nutritional nutrient database for standard reference. Release 24, 2011. www.ars.usda.gov/ SP2UserFiles/Place/12354500/Data/SR24/ sr24_doc.pdf (accessed June 12, 2013).

257 World Cancer Research Fund, American Institute for Cancer Research: Food, Nutrition, Physical Activity, and the Prevention of Cancer: A Global Perspective. Washington, 2007. 
258 World Cancer Research Fund, American Institute for Cancer Research: WCRF/AICR systematic literature review continuous update report. The associations between food, nutrition and physical activity and the risk of breast cancer. 2008. http://www.dietand cancerreport.org/cancer_resource_center/ downloads/cu/Breast-Cancer-SLR-2008. pdf (accessed March 5, 2014).

259 World Cancer Research Fund, American Institute for Cancer Research: WCRF/AICR systematic literature review continuous update project report. The associations between food, nutrition and physical activity and the risk of colorectal cancer. 2010. http://www. dietandcancerreport.org/cancer_resource_ center/downloads/cu/Colorectal-CancerSLR-2010.pdf (accessed March 5, 2014).

260 World Cancer Research Fund, American Institute for Cancer Research: WCRF/AICR systematic literature review continuous update project report. The associations between food, nutrition and physical activity and the risk of pancreatic cancer. 2011. http://www.dietandcancerreport.org/ cancer_resource_center/downloads/cu/ Pancreatic-Cancer-SLR-2011.pdf (accessed March 5, 2014).

261 World Cancer Research Fund, American In- 269 stitute for Cancer Research: WCRF/AICR systematic literature review continuous update project. The associations between food, nutrition and physical activity and the risk of endometrial cancer. 2012. http://www. dietandcancerreport.org/cancer_resource_ center/downloads/cu/Endometrial-CancerSLR-2012.pdf (accessed March 5, 2014).

262 Prentice RL, Thomson CA, Caan B, Hubbell FA, Anderson GL, Beresford SA, Pettinger M, Lane DS, Lessin L, Yasmeen S, Singh B, Khandekar J, Shikany JM, Satterfield S, Chlebowski RT: Low-fat dietary pattern and cancer incidence in the Women's Health Initiative Dietary Modification Randomized Controlled Trial. J Natl Cancer Inst 2007;99: 1534-1543.

263 Liu L, Zhuang W, Wang RQ, Mukherjee R, Xiao SM, Chen Z, Wu XT, Zhou Y, Zhang HY: Is dietary fat associated with the risk of colorectal cancer? A meta-analysis of 13 prospective cohort studies. Eur J Nutr 2011;50: 173-184.

264 Dahm CC, Keogh RH, Lentjes MA, Spencer EA, Key TJ, Greenwood DC, Cade JE, Burley VJ, Shipley MJ, Brunner EJ, Stephen AM, Mishra G, Kuh D, Fentiman IS, White IR, Luben R, Khaw KT, Rodwell Bingham SA: Intake of dietary fats and colorectal cancer risk: prospective findings from the UK dietary cohort consortium. cancer epidemiol 2010;34:562-567.

265 World Cancer Research Fund, American Institute for Cancer Research: Food, Nutrition and the Prevention of Cancer: a Global Perspective. Washington, 1997.

266 Smith-Warner SA, Ritz J, Hunter DJ, Albanes D, Beeson WL, van den Brandt PA,
Colditz G, Folsom AR, Fraser GE, Freudenheim JL, Giovannucci E, Goldbohm RA, Graham S, Kushi LH, Miller AB, Rohan TE, Speizer FE, Virtamo J, Willett WC: Dietary fat and risk of lung cancer in a pooled analysis of prospective studies. Cancer Epidemiol Biomarkers Prev 2002;11:987-992.

267 Allen NE, Appleby PN, Key TJ, Bueno-deMesquita HB, Ros MM, Kiemeney LA, Tjønneland A, Roswall N, Overvad K, Weikert S, Boeing H, Chang-Claude J, Teucher B, Panico S, Sacerdote C, Tumino R, Palli D, Sieri S, Peeters P, Quirós JR, Jakszyn P, Molina-Montes E, Chirlaque MD, Ardanaz E, Dorronsoro M, Khaw KT, Wareham N, Ljungberg B, Hallmans G, Ehrnström R, Ericson U, Gram IT, Parr CL, Trichopoulou A, Karapetyan T, Dilis V, Clavel-Chapelon F, Boutron-Ruault MC, Fagherrazzi G, Romieu I, Gunter MJ, Riboli E: Macronutrient intake and risk of urothelial cell carcinoma in the European prospective investigation into cancer and nutrition. Int J Cancer 2013;132:635-644.

268 Arem H, Mayne ST, Sampson J, Risch H, Stolzenberg-Solomon RZ: Dietary fat intake and risk of pancreatic cancer in the prostate, lung, colorectal and ovarian cancer screening trial. Ann Epidemiol 2013;23:571-575.

69 Turner LB: A meta-analysis of fat intake, reproduction, and breast cancer risk: an evolutionary perspective. Am J Hum Biol 2011; 23:601-608.

270 Park SY, Kolonel LN, Henderson BE, Wilkens LR: Dietary fat and breast cancer in postmenopausal women according to ethnicity and hormone receptor status: the Multiethnic Cohort Study. Cancer Prev Res (Phila) 2012;5:216-228.

271 Sczaniecka AK, Brasky TM, Lampe JW, Patterson RE, White E: Dietary intake of specific fatty acids and breast cancer risk among postmenopausal women in the VITAL cohort. Nutr Cancer 2012;64:1131-1142.

-272 Key TJ, Appleby PN, Cairns BJ, Luben R, Dahm CC, Akbaraly T, Brunner EJ, Burley V, Cade JE, Greenwood DC, Stephen AM, Mishra G, Kuh D, Keogh RH, White IR, Bhaniani A, Borgulya G, Mulligan AA, Khaw KT: Dietary fat and breast cancer: comparison of results from food diaries and food-frequency questionnaires in the UK dietary cohort consortium. Am J Clin Nutr 2011;94:1043-1052.

273 Crane TE, Khulpateea BR, Alberts DS, Basen-Engquist K, Thomson CA: Dietary intake and ovarian cancer risk: a systematic review. Cancer Epidemiol Biomarkers Prev 2014;23:255-273.

274 Blank MM, Wentzensen N, Murphy MA, Hollenbeck A, Park Y: Dietary fat intake and risk of ovarian cancer in the NIH-AARP Diet and Health Study. Br J Cancer 2012;106: 596-602.

275 Chang ET, Lee VS, Canchola AJ, Clarke CA, Purdie DM, Reynolds P, Anton-Culver H, Bernstein L, Deapen D, Peel D, Pinder R, Ross RK, Stram DO, West DW, Wright W, Ziogas A, Horn-Ross PL: Diet and risk of ovarian cancer in the California Teachers Study cohort. Am J Epidemiol 2007;165:802-813.

276 Crowe FL, Key TJ, Appleby PN, Travis RC, Overvad K, Jakobsen MU, Johnsen NF, Tjønneland A, Linseisen J, Rohrmann S, Boeing H, Pischon T, Trichopoulou A, Lagiou P, Trichopoulos D, Sacerdote C, Palli D, Tumino R, Krogh V, Bueno-de-Mesquita HB, Kiemeney LA, Chirlaque MD, Ardanaz E, Sánchez MJ, Larrañaga N, González CA, Quirós JR, Manjer J, Wirfält E, Stattin P, Hallmans G, Khaw KT, Bingham S, Ferrari P, Slimani N, Jenab M, Riboli E: Dietary fat intake and risk of prostate cancer in the European prospective investigation into cancer and nutrition. Am J Clin Nutr 2008; 87:1405-1413.

277 Pelser C, Mondul AM, Hollenbeck AR, Park Y: Dietary fat, fatty acids, and risk of prostate cancer in the NIH-AARP Diet and Health Study. Cancer Epidemiol Biomarkers Prev 2013;22:697-707.

278 Bassett JK, Severi G, Hodge AM, Macinnis RJ, Gibson RA, Hopper JL, English DR, Giles GG: Plasma phospholipid fatty acids, dietary fatty acids and prostate cancer risk. Int J Cancer 2013;133:1882-1891.

279 Bandera EV, Kushi LH, Moore DF, Gifkins DM, McCullough ML: Dietary lipids and endometrial cancer: the current epidemiologic evidence. Cancer Causes Control 2007;18: 687-703.

280 Cui X, Rosner B, Willett WC, Hankinson SE: Dietary fat, fiber, and carbohydrate intake in relation to risk of endometrial cancer. Cancer Epidemiol Biomarkers Prev 2011;20: 978-989.

281 Crowe FL, Allen NE, Appleby PN, Overvad K, Aardestrup IV, Johnsen NF, Tjønneland A, Linseisen J, Kaaks R, Boeing H, Kröger J, Trichopoulou A, Zavitsanou A, Trichopoulos D, Sacerdote C, Palli D, Tumino R, Agnoli C, Kiemeney LA, Bueno-de-Mesquita $\mathrm{HB}$, Chirlaque MD, Ardanaz E, Larrañaga N, Quirós JR, Sánchez MJ, González CA, Stattin P, Hallmans G, Bingham S, Khaw KT, Rinaldi S, Slimani N, Jenab M, Riboli E, Key TJ: Fatty acid composition of plasma phospholipids and risk of prostate cancer in a casecontrol analysis nested within the European prospective investigation into cancer and nutrition. Am J Clin Nutr 2008;88:1353-1363.

282 Dahm CC, Gorst-Rasmussen A, Crowe FL, Roswall N, Tjønneland A, Drogan D, Boeing H, Teucher B, Kaaks R, Adarakis G, Zylis D, Trichopoulou A, Fedirko V, Chajes V, Jenab M, Palli D, Pala V, Tumino R, Ricceri F, van Kranen H, Bueno-de-Mesquita HB, Quirós JR, Sánchez MJ, Luján-Barroso L, Larrañaga N, Chirlaque MD, Ardanaz E, Johansson M, Stattin P, Khaw KT, Wareham N, Wark PA, Norat T, Riboli E, Key TJ, Overvad K: Fatty acid patterns and risk of prostate cancer in a case-control study nested within the European Prospective Investigation into Cancer and Nutrition. Am J Clin Nutr 2012; 96:1354-1361. 
283 Park SY, Wilkens LR, Henning SM, Le Marchand L, Gao K, Goodman MT, Murphy SP, Henderson BE, Kolonel LN: Circulating fatty acids and prostate cancer risk in a nested case-control study: the Multiethnic Cohort. Cancer Causes Control 2009;20:211223.

284 Daniel CR, McCullough ML, Patel RC, Jacobs EJ, Flanders WD, Thun MJ, Calle EE: Dietary intake of omega- 6 and omega- 3 fatty acids and risk of colorectal cancer in a prospective cohort of U.S. men and women. Cancer Epidemiol Biomarkers Prev 2009; 18: 516-525.

285 Butler LM, Wang R, Koh WP, Stern MC, Yuan JM, Yu MC: Marine n-3 and saturated fatty acids in relation to risk of colorectal cancer in Singapore Chinese: a prospective study. Int J Cancer 2009;124:678-686.

-286 Sasazuki S, Inoue M, Iwasaki M, Sawada N, Shimazu T, Yamaji T, Takachi R, Tsugane S: Intake of n-3 and n- 6 polyunsaturated fatty acids and development of colorectal cancer by subsite: Japan Public Health Centerbased prospective study. Int J Cancer 2011; 129:1718-1729.

287 Chua ME, de Sio MC, Sorongon MC, Dy JS: Relationship of dietary intake of omega-3 and omega- 6 fatty acids with risk of prostate cancer development: a meta-analysis of prospective studies and review of literature. Prostate Cancer 2012;2012:826254.

-288 Sakai M, Kakutani S, Horikawa C, Tokuda $\mathrm{H}$, Kawashima H, Shibata H, Okubo H, Sasaki S: Arachidonic acid and cancer risk: a systematic review of observational studies. BMC Cancer 2012;12:606.
289 Gerber M: Omega-3 fatty acids and cancers: a systematic update review of epidemiological studies. Br J Nutr 2012;107(suppl 2): S228-S239.

290 Shen XJ, Zhou JD, Dong JY, Ding WQ, Wu JC: Dietary intake of n-3 fatty acids and colorectal cancer risk: a meta-analysis of data from 489000 individuals. Br J Nutr 2012;108:1550-1556.

291 Zheng JS, Hu XJ, Zhao YM, Yang J, Li D: Intake of fish and marine n-3 polyunsaturated fatty acids and risk of breast cancer: meta-analysis of data from 21 independent prospective cohort studies. BMJ 2013;346: f3706.

292 Hall MN, Chavarro JE, Lee IM, Willett WC, Ma J: A 22-year prospective study of fish, n-3 fatty acid intake, and colorectal cancer risk in men. Cancer Epidemiol Biomarkers Prev 2008; 17:1136-1143.

293 Qin B, Xun P, He K: Fish or long-chain (n-3) PUFA intake is not associated with pancreatic cancer risk in a meta-analysis and systematic review. J Nutr 2012;142:1067-1073.

294 Yang B, Ren XL, Fu YQ, Gao JL, Li D: Ratio of n-3/n-6 PUFAs and risk of breast cancer: a meta-analysis of 274135 adult females from 11 independent prospective studies. BMC Cancer 2014;14:105.

295 Leitzmann MF, Stampfer MJ, Michaud DS, Augustsson K, Colditz GC, Willett WC, Giovannucci EL: Dietary intake of n-3 and $\mathrm{n}-6$ fatty acids and the risk of prostate cancer. Am J Clin Nutr 2004;80:204-216.
296 Koralek DO, Peters U, Andriole G, Reding D, Kirsh V, Subar A, Schatzkin A, Hayes R, Leitzmann MF: A prospective study of dietary alpha-linolenic acid and the risk of prostate cancer (United States). Cancer Causes Control 2006;17:783-791.

297 Brasky TM, Darke AK, Song X, Tangen CM, Goodman PJ, Thompson IM, Meyskens FL Jr, Goodman GE, Minasian LM, Parnes HL, Klein EA, Kristal AR: Plasma phospholipid fatty acids and prostate cancer risk in the SELECT trial. J Natl Cancer Inst 2013;105: 1132-1141.

298 Brasky TM, Till C, White E, Neuhouser ML, Song X, Goodman P, Thompson IM, King IB, Albanes D, Kristal AR: Serum phospholipid fatty acids and prostate cancer risk: results from the prostate cancer prevention trial. Am J Epidemiol 2011;173: 1429-1439.

299 Deutsche Gesellschaft für Ernährung, Österreichische Gesellschaft für Ernährung, Schweizerische Gesellschaft für Ernährung: Referenzwerte für die Nährstoffzufuhr, ed 2. Bonn, 2015

300 Deutsche Gesellschaft für Ernährung e.V.: Vollwertig essen und trinken nach den 10 Regeln der DGE, ed 25. Bonn, 2013.

301 Stehle P, Oberritter H, Büning-Fesel M, Heseker H: Grafische Umsetzung von Ernährungsrichtlinien - traditionelle und neue Ansätze. Ernährungs Umschau 2005; 52:128-135.

302 Jungvogel A, Wendt I, Schäbethal K, Leschik-Bonnet E, Oberritter H: Überarbeitet: Die 10 Regeln der DGE. Ernährungs Umschau 2013;59:M644-M645. 